\title{
Mirror symmetry, mirror map and applications to complete intersection Calabi-Yau spaces
}

\author{
S. Hosono ${ }^{\mathrm{a}, 1}$, A. Klemm ${ }^{\mathrm{b}, \mathrm{c}, 2}$, S. Theisen ${ }^{\mathrm{d}, 3}$, S.-T. Yau ${ }^{\mathrm{c}, 4}$ \\ ${ }^{a}$ Department of Mathematics, Toyama University, Toyama 930, Japan \\ ${ }^{\mathrm{b}}$ CERN, TH-Division, CH-1211 Geneva 23, Switzerland \\ ${ }^{c}$ Department of Mathematics, Harvard University, Cambridge, MA 02138, USA \\ ${ }^{d}$ Sektion Physik der Universität München Theresienstraße 37, D-80333 Munich, FRG
}

Received 17 June 1994; accepted 29 September 1994

\begin{abstract}
We extend the discussion of mirror symmetry, Picard-Fuchs equations, instanton corrected Yukawa couplings and the topological one-loop partition function to the case of complete intersections with higher dimensional moduli spaces. We will develop a new method of obtaining the instanton corrected Yukawa couplings through a study of the solutions of the Picard-Fuchs equations. This leads to closed formulas for the prepotential for the Kähler moduli fields induced from the ambient space for all complete intersections in nonsingular weighted projective spaces. As examples we treat part of the moduli space of the phenomenologically interesting three-generation models which are found in this class. We also apply our method to solve the simplest model in which a topology change was observed and discuss examples of complete intersections in singular ambient spaces.
\end{abstract}

\section{Introduction}

Complete intersection Calabi-Yau manifolds embedded in products of projective spaces (CICY) are the most prominent candidates for the compactification of the heterotic string. They have been intensively studied. Let us point out briefly the main results; a detailed account from a physical point of view can be found in

\footnotetext{
${ }^{1}$ E-mail: hosono@nqs07.ccis.toyama-u.ac.jp.

${ }^{2}$ E-mail: klemm@nxth21.cern.ch.

${ }^{3}$ E-mail: theisen@mppmu.mpg.de.

${ }^{4}$ E-mail: yau@harvard.math.edu.
} 
[1]. Using the $c_{1}=0$ condition and curve identities it was recognized in [2] that all CICYs can be described by finitely many configurations of polynomials in products of projective spaces. Each configuration leads to a family of Calabi-Yau spaces whose generic member is smooth. By a computer classification 7868 configurations with Euler numbers between -200 and 0 were found in [3,4]. In [5] all their Hodge numbers were calculated. There occur 265 different combinations. Application of a theorem of Wall [6], which states that the homotopy types of Calabi-Yau threefolds $\mathrm{X}$ can be classified by their Hodge numbers, their topological triple couplings $K_{i j k}^{0} \equiv \int_{\mathrm{X}} J_{i} \wedge J_{j} \wedge J_{k}$ and $c_{2} \cdot J_{i} \equiv \int_{\mathrm{X}} c_{2} \wedge J_{i}$, reveals that there are at least 2590 topologically different models in this class [7]. Green and Huübsch have shown in [8] that all families of CICYs are connected by the process, already described in [3], of contracting a family to a nodal configuration and performing a small resolution of the latter. Certain quotients of them by discrete groups were constructed in $[9,10]$ which have Euler number $\chi=-6$. Two of them, discussed below, have a nontrivial fundamental group $\left(\pi_{1}(X)=\mathbb{Z}_{3}\right)$ and give rise to heterotic string compactifications with three generations and a natural option to break the $\mathrm{E}_{6}$ gauge group by Wilson lines.

In this paper we want to extend the analysis of the Picard-Fuchs equations, the construction of the mirror map and the calculation of the instanton corrected Yukawa couplings, which was performed for one-moduli hypersurfaces in a (weighted) projective space in [11-14] and generalized to higher dimensional moduli spaces of general hypersurfaces in toric varieties in $[15,16]$ to the class of complete intersections in products of weighted projective spaces. It is natural to focus on the derivation of the prepotentials for the complex structure and the Kähler structure deformations, which encode all information of these two (topological) subsectors of the theory at tree level. The knowledge of the prepotentials is quite relevant for the low energy phenomenology of the subsector of the moduli and the $\mathbf{2 7}, \overline{27}$ matter fields in the effective field theory.

We will show that there is an easily exactly solvable subsystem of the complex structure deformations, namely the one which is, by the mirror map, associated to the quantum cohomology of the subset of the elements in $H^{1,1}(X, \mathbb{Z})$ induced from the Kähler forms of the projective spaces. We focus on this subsystem and present results in a closed form for the instanton corrected cohomology of this system. In general the moduli space is enlarged due to exceptional divisors coming from the resolution of possible singularities. This situation we have treated quite generally for hypersurfaces in [16]. Here we will give two simple but illustrative examples how to deal with the exceptional divisors in the complete intersection case.

Even in the smooth case there are usually more elements in $H^{1,1}(X, \mathbb{Z})$ than the ones mentioned above. For the counting and the calculation of intersections of the latter see [1]. If they correspond under the mirror map to complex structure deformations, which can be represented as deformations of the polynomials by vector monomials, they can in principle be incorporated. This is e.g. the situation for the Tian-Yau manifold. If a Landau-Ginzburg prescription of the model is available, also perturbations which are not of this kind can be represented as roots of monomial deformations, as was suggested in [17]. 
Our approach covers all cases treated in [11-14,18-20]. Due to threefold isomorphisms, it also covers the two two-moduli Calabi-Yau spaces which were treated in [15] and among others in [16], namely the degree- 8 hypersurface in $\mathbb{P}^{4}[2,2,2,1,1]$ and the degree- 12 hypersurface in $\mathbb{P}^{4}[6,2,2,1,1]$. We will see moreover that the described subsystems provide simple examples of higher dimensional Calabi-Yau moduli spaces, the simplest case being the subsystem of the Tian-Yau manifold. Apart from being phenomenologically interesting, there exists also a detailed mathematical study on the rational curves of this manifold [21].

We organize the material as follows. In Section 2 we shortly review how to calculate classical intersections numbers. In the third section we explain our method of deriving the system of Picard-Fuchs equations. In Section 4 we exhibit the general structure of the solutions of the Picard-Fuchs equations. This, when combined with the mirror hypothesis, leads to the main result of this section, namely a concise formula for the prepotential. This solves the problem of determining the moduli dependence of the instanton corrected Yukawa couplings and the Weil-Peterson metric for the states associated to the Kähler forms of the ambient space for the class of $(2,2)$ compactifications on CICYs in the large radius limit completely ${ }^{5}$.

We demonstrate our method with some selected examples in Section 5. In Section 6 we discuss the connection of certain CICY manifolds with rational superconformal field theories. This way we can also explain the occurrence of identical invariants for the rational and elliptic curves on some pairs of hypersurfaces and complete intersections by an identity of conformal field theories. Following the approach of [22] we extend in Section 7 the analysis to the topological one-loop partition function, which is a moduli dependent quantity describing the difference between the threshold corrections to the $E_{8}$ and $E_{6}$ gauge couplings [23] (see also [24]). The expansion of the one-loop partition function has a conjectural interpretation in terms of Gromov-Witten invariants for elliptic curves. As the geometrical understanding of these predictions is in a somewhat preliminary state, we found it useful to use our data to calculate them explicitly for various one-, two- and three-moduli examples of different type. In the final section we discuss some open problems and possible avenues for future work.

\section{Calculation of the classical topological data of CICYs}

In the following we consider complete intersections of $l$ hypersurfaces in products of $k$ projective spaces. Since most formulas allow for an incorporation of weights we will state them for the general case. Denote by $d_{j}^{(i)}$ the degree of the

\footnotetext{
${ }^{5}$ Instead of presenting a lengthy list of examples, we distribute the Mathematica program INSTANTON which calculates the Yukawa couplings and counts the numbers of rational curves for any complete intersection Calabi-Yau manifold discussed in $[3,4]$ and other examples in singular ambient spaces. It is appended to the hep-th/9406055 version of this paper.
} 
coordinates of $\mathbb{P}^{n_{i}}\left[\boldsymbol{w}^{(i)}\right]$ in the $j$ th polynomial $p_{j}(i=1, \ldots, k ; j=1, \ldots, l)$. We will use the notation

$$
\left(\begin{array}{c}
\mathbb{P}^{n_{1}}\left[w_{1}^{(1)}, \ldots, w_{n_{1}+1}^{(1)}\right] \\
\vdots \\
\mathbb{P}^{n_{k}}\left[w_{1}^{(k)}, \ldots, w_{n_{k}+1}^{(k)}\right]
\end{array} \| \begin{array}{ccc}
d_{1}^{(1)} & \ldots & d_{l}^{(1)} \\
\vdots & & \vdots \\
d_{1}^{(k)} & \ldots & d_{l}^{(k)}
\end{array}\right)_{\chi}^{h^{1,1}}
$$

for a configuration. Let us associate to the Kähler forms induced from the $i$ th projective space the formal variable $J_{i}$ and consider the map $\Pi: \mathbb{Z}\left[J_{1}, \ldots, J_{k}\right] \rightarrow \mathbb{Z}$ defined on the generators $J_{s}$ as

$$
\begin{aligned}
\Pi\left(J_{s}\right)= & \left(\prod_{r=1}^{k} \frac{\partial_{J_{r}}^{n_{r}}}{n_{r} !}\right)\left(\frac{\prod_{i=1}^{k} \prod_{j=1}^{n_{i}+1}\left(1+w_{j}^{(i)} J_{i}\right)}{\prod_{j=1}^{l}\left(1+\Sigma_{i=1}^{k} d_{j}^{(i)} J_{i}\right)}\right) \\
& \times\left.\left(\frac{\prod_{j=1}^{l} \sum_{i=1}^{k} d_{j}^{(i)} J_{i}}{\prod_{i=1}^{k} \prod_{j=1}^{n_{i}+1} w_{j}^{(i)}}\right) J_{s}\right|_{J_{1}=\ldots=J_{k}=0} .
\end{aligned}
$$

It follows from the adjunction formula that the term in the second bracket on the right-hand side yields, by formal expansion, the total Chern class

$$
c(\mathrm{X})=1+c_{1}+c_{2}+c_{3}=1+c_{1}^{a} J_{a}+c_{2}^{a b} J_{a} J_{b}+c_{3}^{a b c} J_{a} J_{b} J_{c}
$$

of the Calabi-Yau manifold X. The coefficients are given by

$$
\begin{aligned}
& c_{1}^{a}=\sum_{i=1}^{n_{a}+1} w_{i}^{(a)}-\sum_{i=1}^{l} d_{i}^{(a)} \equiv 0, \\
& c_{2}^{a b}=\frac{1}{2}\left(-\delta^{a b} \sum_{i=1}^{n_{a}+1}\left(w_{i}^{(a)}\right)^{2}+\sum_{i=1}^{l} d_{i}^{(a)} d_{i}^{(b)}\right), \\
& c_{3}^{a b c}=\frac{1}{3}\left(\delta^{a b c} \sum_{i=1}^{n_{a}+1}\left(w_{i}^{(a)}\right)^{3}-\sum_{i=1}^{l} d_{i}^{(a)} d_{i}^{(b)} d_{i}^{(c)}\right) .
\end{aligned}
$$

Here we have enforced the vanishing of the first Chern class. The numerator of the third term in (2.2) is the top Chern class of the normal bundle of $\mathrm{X}$ and the denominator is a normalization of the volume of the weighted projective space. Applying $\Pi$ to a monomial of the $J_{i}$ is equivalent to the integration of the wedge product of the corresponding $(1,1)$ forms, also denoted by $J_{i}$, wedged with the Chern class of dual form degree over $\mathrm{X}$. We have therefore

$$
\begin{aligned}
& \chi=\int_{\mathrm{X}} c_{3}=\Pi(1), \quad \int_{\mathrm{X}} c_{2} \wedge J_{m}=\Pi\left(J_{m}\right), \\
& K_{i j k}^{0}=\int_{\mathrm{X}} J_{i} \wedge J_{j} \wedge J_{k}=\Pi\left(J_{i} J_{j} J_{k}\right) .
\end{aligned}
$$


Note that these formulas, with the exception of the first line in (2.4), are valid only for the case in which $\mathrm{X}$ has no singularities. For the singular case the triple intersection gets modified to $\int_{\mathrm{X}} h_{i} \wedge h_{j} \wedge h_{k}=\Pi\left(J_{i} J_{j} J_{k}\right) n_{0}^{(i)} n_{0}^{(j)} n_{0}^{(k)}$, where $n_{0}^{(i)}$ is the least common multiple of the orders of the isotropy groups of all fixed points of the manifold under $\mathbb{C}^{*}$ actions in the $i$ th weighted projective space. The modification of the first two integrals is more involved. E.g. for complete intersections in one weighted projective space one has [25]

$$
\int_{\mathrm{X}} c_{2} \wedge J=\left.\frac{12}{n_{0} !}\left(\frac{\partial}{\partial J}\right)^{n_{0}} \frac{\Pi_{i=1}^{l}\left(1-J^{d_{i}}\right)}{\prod_{i=1}^{n+1}\left(1-J^{w_{i}}\right)}\right|_{J=0}-2 \int_{\mathrm{X}} J \wedge J \wedge J .
$$

In Section 4 we will provide a more illuminative generalization of the formulas (2.4) to the case of hypersurfaces and complete intersections with desingularized quotient singularities.

For example, in a product of two (weighted) projective spaces we have a smooth configuration

$$
\left(\begin{array}{c}
\mathbb{P}^{4}[3,1,1,1,1] \\
\mathbb{P}^{1}
\end{array} \| \begin{array}{cc}
6 & 1 \\
0 & 2
\end{array}\right)_{-252}^{2} \quad c_{2} \cdot J_{1}=42, \quad c_{2} \cdot J_{2}=24,
$$

where we use the notation of [16] to display the intersection numbers.

\section{Derivation of the Picard-Fuchs equations}

We will begin this section by deriving some general results on the Picard-Fuchs equations as they apply to the models considered here, using the toric data of the manifolds. This approach gives one period by explicit integration and a holonomic system of linear differential operators, which are satisfied by all periods, but which allows for additional solutions. Among the finitely many solutions the periods can be singled out by the requirement that the monodromy acts irreducibly on them. Technically the problem of specifying them is solved here by factorizing the differential operators.

Let us first show how the system of Picard-Fuchs equations for a restricted set of $k$ complex structure deformation parameters can be obtained from toric data of the (mirror) manifolds according to [20]. This system of Picard-Fuchs equations is equivalent to the first order Gauss-Main differential system which describes the variation of the Hodge structure of $\mathrm{H}^{3}(\mathrm{X}, \mathbb{Z})$, restricted to the holomorphic and antiholomorphic $(3,0)$ and $(0,3)$ forms and $k(2,1)$ and $(1,2)$ forms. By the mirror map we will identify the $k$ complex structure deformations with the Kähler deformations in the restricted basis of Kähler forms specified in the previous section.

Here we consider only configurations in which the complete intersection does not intersect with singular loci of the ambient space $\mathbb{P}^{n_{1}}\left[\boldsymbol{w}^{(1)}\right] \times \ldots \times \mathbb{P}^{n_{k}}\left[\boldsymbol{w}^{(k)}\right]$. Without further restricting the generality we may choose $w_{n_{i}+1}^{(i)}=1$ for all $i$. Each projective space $\mathbb{P}^{n_{i}}\left[\boldsymbol{w}^{(i)}\right]$ is a toric variety which can be described by a (reflexive) 
simplicial polyhedron $\Delta_{i}$ with integral vertices in $\mathbb{R}^{n_{i}}$ (see $[26,16]$ for its determination).

Since the ambient space is a direct product of all $\mathbb{P}^{n_{i}}\left[\boldsymbol{w}^{(i)}\right]$, it is also a toric variety described by the reflexive polyhedra $\Delta=\Delta_{1} \times \ldots \times \Delta_{k}$ in $\mathbb{R}^{n_{1}} \times \ldots \times \mathbb{R}^{n_{k}}$.

In $[20,27]$ it was conjectured that the mirror manifold of the CICY of type (2.1) is given by the CICY constructed from the combinatorial data of the data of the dual polyhedron $\Delta^{*}$ of $\Delta . \Delta$ is reflexive and the corners of the dual $\Delta^{*}$ are the integral points $\nu_{i, 1}^{*}=(1,0, \ldots, 0), \ldots, \nu_{i, n_{i}}^{*}=(0, \ldots, 0,1)$ and $\nu_{i, n_{i}+1}^{*}=$ $\left(-w_{1}^{(1)}, \ldots,-w_{n_{i}}^{(i)}\right)$ in $\mathbb{R}^{n_{i}}$ of $\mathbb{R}^{n_{1}} \times \ldots \times \mathbb{R}^{n_{k}}$. These vertices satisfy the relations $\sum_{j=1}^{n_{i}+1} w_{j}^{(i)} \nu_{i, j}^{*}=0(i=1, \ldots, k)$. We group the vertices $\nu_{i, j}^{*}$ into $l$ (= number of defining polynomials) sets (a so-called as nef-partition)

$$
\left\{\nu_{i, j}^{*}\right\}_{1 \leqslant i \leqslant k, 1 \leqslant j \leqslant n_{i}+1}=\mathrm{E}_{1} \cup \ldots \cup \mathrm{E}_{l}
$$

by defining $\mathrm{E}_{m}(1 \leqslant m \leqslant l)$ so that it contains $d_{m}^{(i)}$ vertices from $\left\{\nu_{i, j}^{*}\right\}_{1 \leqslant j \leqslant n_{i}+1}$ for each $i=1, \ldots, k$. We extend each vertex $\nu_{i, j}^{*}$ of $\mathrm{E}_{m}$ to $\bar{\nu}_{i, j}^{*}=\left(e^{(m)}, \nu_{i, j}\right)$ in $\mathbb{R}^{l} \times \mathbb{R}^{n_{1}}$ $\times \ldots \times \mathbb{R}^{n_{k}}$ with $\boldsymbol{e}^{(m)}$ being the unit vector in the $m$ th direction of $\mathbb{R}^{l}$. After adding the additional vertices $\bar{\nu}_{0, p}^{*}=\left(e^{(p)}, 0\right)$, one finds, as a consequence of the first relation in (2.4), $k$ independent linear relations between the $\sum_{i=1}^{l}\left(n_{i}+2\right)$ vertices $\bar{\nu}_{i, j}^{*}$ of the form

$$
\sum l^{(s)} \bar{\nu}_{i, j}^{*}=0 \text {. }
$$

The $l^{(s)}(s=1, \ldots, k ; j=1, \ldots, l)$ are given by

$$
l^{(s)}=\left(-d_{1}^{(s)}, \ldots,-d_{l}^{(s)} ; \ldots, 0, w_{1}^{(s)}, \ldots, w_{n_{s}+1}^{(s)}, 0, \ldots\right) \equiv\left(\left\{l_{0 j}^{(s)}\right\} ;\left\{l_{i}^{(s)}\right\}\right) .
$$

The mirror manifold $X^{*}$ can then be written conjectually as the complete intersection of the vanishing locus of the following Laurent polynomials in the variables $X_{m, n}, m=1, \ldots, k ; n=1, \ldots, n_{m}$ (using the notation of [16]),

$$
P_{r}=a_{r}-\sum_{\nu_{i, j}^{*} \in \mathrm{E}_{r}} a_{i, j} X^{v_{i, j}^{*}} \quad(r=1, \ldots, l),
$$

where the sum is over the (unextended) vertices in the $m$ th set $\mathrm{E}_{m}$. The vanishing loci of (3.4) are considered in a toric variety $\mathbb{P}_{\Delta_{(1)}^{*}+\ldots+\Delta_{(i)}^{*}}$ with $\Delta_{(i)}^{*}$ being a convex hull of $\{0\}$ and the set $E_{i}$. In [27] the combinatorial aspects of this construction have been nicely formulated.

Choosing a cycle $\Gamma_{0}$ determined by $\left|X_{m, n}\right|=1 \forall m, n$, the corresponding period integral (see $[26,16])$,

$$
w_{0}(a)=\int_{\Gamma_{0}} \frac{a_{1} \cdots a_{l}}{P_{1} \cdots P_{l}} \prod_{m=1}^{k} \prod_{n=1}^{n_{m}} \frac{\mathrm{d} X_{m, n}}{X_{m, n}},
$$

can be performed explicitly by expanding the integrand in a multiple power series in the $1 / a_{m}$ and using the residue formula. After introducing the variables

$$
z_{s}=\frac{a_{s, 1}^{w^{(s)}} \cdots a_{s, n+1}^{w_{n}^{(s)}}}{a_{1}^{d^{(s)}} \cdots a_{l}^{d^{(s)}}} \equiv a^{l^{(s)}} \quad(s=1, \ldots, k)
$$


it can be easily verified that the period $w_{0}(a)$ is given by ${ }^{6}$

$$
w_{0}(z)=\sum_{n_{s} \geqslant 0} c(n) z^{n}
$$

with

$$
c(n)=\frac{\prod_{j}\left(-\sum_{s=1}^{k} l_{o j}^{(s)} n_{s}\right) !}{\prod_{i}\left(\sum_{s} l_{i}^{(s)} n_{s}\right) !}=\frac{\prod_{j=1}^{l}\left(\sum_{i=1}^{k} n_{i} d_{j}^{(i)}\right) !}{\prod_{i=1}^{k} \prod_{j=1}^{n_{i}+1}\left(w_{j}^{(i)} n_{i}\right) !} .
$$

It satisfies the generalized hypergeometric system of Gelfand-Kapranov-Zelevinsky with the $k$ linear differential operators

$$
\begin{aligned}
\mathscr{L}_{s}= & \prod_{j=1}^{n_{s}+1}\left(w_{j}^{(s)} \theta_{s}\right)\left(w_{j}^{(s)} \theta_{s}-1\right) \cdots\left(w_{j}^{(s)} \theta_{s}-w_{j}^{(s)}+1\right) \\
& -\prod_{j=1}^{l}\left(\sum_{i=1}^{k} d_{j}^{(l)} \theta_{i}\right) \cdots\left(\sum_{i=1}^{k} d_{j}^{(i)} \theta_{i}-d_{j}^{(s)}+1\right) z_{s}
\end{aligned}
$$

associated to the vector of the coefficients of the linear relations $l^{(s)}, s=1, \ldots, k$, given in (3.3). Here the $\theta_{i}$ are logarithmic derivatives $\theta_{i}=z_{i} \partial / \partial z_{i}$. Similarly as in [16] one can show that these equations are satisfied for all periods $w_{j}$ as they reflect the infinitesimal symmetries of (3.5), independent of the cycle chosen. This system is holonomic, which means that the left ideal $I$ generated by (3.9) in the ring of linear partial differential operators $D$ is of finite $\operatorname{rank} \operatorname{rk}(I)$. This implies the existence of $\operatorname{rk}(I)$ independent solutions [28], where $\operatorname{rk}(I)$ is always larger than the expected number of periods.

We are interested in a subset of solutions of the system (3.9) which corresponds to period integrals over the $2 k+2$ cycles dual to the restricted basis of $\mathrm{H}^{3}(\mathrm{X}, \mathbb{Z})$. These solutions can be characterized by the requirement that the monodromy acts irreducibly on them [29]. Solving the Riemann-Hilbert problem for the reduced monodromy leads to a reduced system of (lower degree) differential operators $L_{1}, \ldots, L_{h} . h$ denotes the number of Picard-Fuchs equations (cf. below). In many examples such a system can be specified by factorizing differential operators from (3.9) in the form $p_{i}(\theta) L_{i}:=\sum_{j=1}^{k} q_{j}(\theta) \mathscr{L}_{j}$, where $p_{i}(\theta)$ and $q_{j}(\theta)$ are polynomials in $\theta$. We remark that this is, however, not the generic situation (see [16]). The system $L_{1}, \ldots, L_{h}$ is again holonomic and generates a left ideal whose rank is $2(k+1)$. In fact this is our criterium to check that a given system of PF equations is complete (cf. below).

Let us demonstrate the derivation of the system $L_{1}, \ldots, L_{h}$ for the following complete intersection Calabi-Yau manifold:

$$
\left(\begin{array}{l||ll}
\mathbb{P}^{3} & 3 & 1 \\
\mathbb{P}^{2} & 0 & 3
\end{array}\right)_{-54}^{8}
$$

\footnotetext{
${ }^{6}$ Here and in the following we denote by $\theta, z, n$ and $\rho$ the $k$-tuples $\theta_{1}, \ldots, \theta_{k}, z_{1} \ldots z_{k}, n_{1}, \ldots, n_{k}$ and $\rho_{1}, \ldots, \rho_{k}$. We use obvious abbreviations such as $z^{n}:=\prod_{s=1}^{k} z_{s}^{n_{s}}, n !:=\prod_{s=1}^{k} n_{s} !$ etc.
} 
The toric description of the mirror manifold was already given in [20]. One has two reflexive simplicial polyhedra $\Delta_{1}^{*}, \Delta_{2}^{*}$ with vertices in $\mathbb{R}^{3} \times \mathbb{R}^{2}$ :

$$
\begin{array}{ll}
\nu_{1,1}^{*}=(1,0,0 ; 0,0), & \nu_{1,2}^{*}=(0,1,0 ; 0,0), \\
\nu_{1,3}^{*}=(0,0,1 ; 0,0), & \nu_{1,4}^{*}=(-1,-1,-1 ; 0,0) ; \\
\nu_{2,1}^{*}=(0,0,0 ; 1,0), & \nu_{2,2}^{*}=(0,0,0 ; 0,1), \quad \nu_{2,3}^{*}=(0,0,0 ;-1,-1) .
\end{array}
$$

We now group the vertices into two sets $E_{1}=\left\{\nu_{1,1}^{*}, \nu_{1,2}^{*}, \nu_{1,3}^{*}\right\}$ and $E_{2}=$ $\left\{\nu_{2,1}^{*}, \nu_{2,2}^{*}, \nu_{2,3}^{*}, \nu_{1,4}^{*}\right\}$ and define the extended vertices $\bar{\nu}^{*}=\left(e_{1,2} ; \nu^{*}\right)$ in $\mathbb{R}^{7}$ where we choose $e_{1}^{(1)}=(1,0)$ and $e^{(2)}=(0,1)$ for the vertices in the first and second sets, respectively. The results derived in the following are independent of how we group the vertices into two sets as long as the first set contains three vertices $\nu_{1, i}^{*}$ and the second set contains the remaining vertices. After adding the two vertices $\nu_{0, k}^{*}=$ $\left(e^{(k)}, \mathbf{0}\right), k=1,2$ this leads by (3.4) to the following two Laurent polynomials:

$$
\begin{aligned}
& P_{1}=a_{1}-a_{1,1} X_{1}-a_{1,2} X_{2}-a_{1,3} X_{3}, \\
& P_{2}=a_{2}-a_{2,1} Y_{1}-a_{2,2} Y_{2}-\frac{a_{2,3}}{Y_{1} Y_{2}}-\frac{a_{1,4}}{X_{1} X_{2} X_{3}} .
\end{aligned}
$$

We now have independent linear relations ${ }^{7} \sum l^{(k)} \bar{\nu}_{i, j}^{*}=0, k=1,2$, between the vertices, namely

$$
l^{(1)}=(-3,-1 ; 1,1,1,1,0,0,0) \quad \text { and } \quad l^{(2)}=(0,-3 ; 0,0,0,0,1,1,1) .
$$

They are of the form (3.3) and define the variables $z_{1}=a_{1,1} a_{1,2} a_{1,3} a_{1,4} / a_{1}^{3} a_{2}$, $z_{2}=a_{2,1} a_{2,2} a_{2,3} / a_{2}^{3}$ via (3.6). The parameters $a_{i, j}$ correspond to trivial automorphisms of (3.12) and can be set to one. Using (3.9) we can associate the following GKZ system to the $l^{(i)}$ :

$$
\begin{aligned}
& \mathscr{L}_{1}=\theta_{1}^{4}-3 \theta_{1}\left(\theta_{1}+3 \theta_{2}\right)\left(3 \theta_{1}-1\right)\left(3 \theta_{1}-2\right) z_{1}, \\
& \mathscr{L}_{2}=\theta_{2}^{3}-\left(\theta_{1}+3 \theta_{2}\right)\left(\theta_{1}+3 \theta_{2}-1\right)\left(\theta_{1}+3 \theta_{2}-2\right) z_{2} .
\end{aligned}
$$

In addition to the power series solution the system has eleven logarithmic ones. To obtain an irreducible subsystem we factorize in the following way: $\mathscr{L}_{1}=: \theta_{1} L_{1}$ and $\mathscr{L}_{1}+27 \mathscr{L}_{2}=: \theta_{1}\left(\theta_{1}+\theta_{2}\right) L_{2}$, which leads to the reduced system

$$
\begin{aligned}
L_{1}= & \theta_{1}^{3}-3\left(\theta_{1}+3 \theta_{2}\right)\left(3 \theta_{1}-1\right)\left(3 \theta_{1}-2\right) z_{1}, \\
L_{2}= & \left(\theta_{1}^{2}-3 \theta_{1} \theta_{2}+9 \theta_{2}^{2}\right)-3\left(3 \theta_{1}-1\right)\left(3 \theta_{1}-2\right) z_{1} \\
& -27\left(\theta_{1}+3 \theta_{2}-1\right)\left(\theta_{1}+3 \theta_{2}-2\right) z_{2} .
\end{aligned}
$$

The fact that the number of equations equals the number of moduli is special to the example here. In fact we will see in example in Section 5.7 that the numbers of linear differential operators describing the Picard-Fuchs system can vary locally in the different patches of the moduli space.

\footnotetext{
${ }^{7}$ The components of the $l^{(k)}$ refer to vertices $\bar{\nu}_{i, j}^{*}$ with $i, j$ in lexicographic order.
} 
Let us finally comment on some generic features of the moduli space of the mirror manifold and its compactification, as far as we will need them for fixing the holomorphic ambiguity in Section 7. It is always easy to find the invariance group of the Laurent polynomials, which acts by phase multiplication on the parameter $a_{i}$ and the coordinates $X_{i}, Y_{i}, \ldots$ In our example one finds a $\mathbb{Z}_{9}$ invariance group of (3.12) acting by $\left(X_{i}, Y_{j}, a_{1}, a_{2}\right) \mapsto\left(\alpha^{k} X_{i}, \alpha^{6 k} Y_{i}, \alpha^{k} a_{1}, \alpha^{6 k} a_{2}\right)$, with $\alpha=\mathrm{e}^{2 \pi i / 9}$ and $k \in \mathbb{Z}$. Because of this invariance of the parameter space of (3.12), we have to define the moduli space of the mirror manifold as the quotient $\mathscr{M}=\mathscr{\mathscr { M }} / \mathbb{Z}_{9}$, where $\tilde{\mathscr{M}}$ is parametrized by $a_{1}, a_{2}$. It is easy to see that the parameters (3.6) are in general invariant under the group action on the Laurent polynomials and hence well defined on the quotient ${ }^{8}$. The fact that (3.6) are invariant under the group action and hence well defined on the quotient can be seen in general ${ }^{8}$.

The singularity of the moduli space of the Laurent polynomials at $a_{i}=0$ due to the phase symmetries can now always be described by toric geometry. In general one has to consider as a second step also the quotient with respect to invariances of the Laurent polynomials which are not acting simply by phase multiplication. In our example such symmetries are not present and we see that $\tilde{n}_{1}=a_{1}^{9}, \tilde{n}_{2}=a_{1}^{3} a_{2}$ and $\tilde{n}_{3}=a_{2}^{3}$ generate the multiplicative semigroup of invariant monomials under the $\mathbb{Z}_{9}$ but satisfy the relation $\tilde{n}_{1} \tilde{n}_{3}=\tilde{n}_{2}^{3}$, describing a rational $\mathrm{A}_{2}$ double point. It is now straightforward to give a toric description of the moduli space using the secondary fan construction of [30]. The secondary fan, whose dimension equals the number of Kähler moduli, contains the Kähler cone. Since it is a complete fan, it gives a toric description of the compactification of Kähler moduli space; for a review, see also [31]. If we define a matrix $B$ whose rows are the generators $l^{(s)}$ of the Mori cone, then the columns of $B$ generate the one-dimensional cones of the secondary fan. Their minimal generators for the model considered here are the vectors $e_{1}, e_{2},-\left(e_{1}+3 e_{2}\right)$ and $-e_{1}$, where $e_{1}, e_{2}$ generate a square lattice. The cone ${ }^{9}\left\langle e_{1}, e_{2}\right\rangle$ is the Kähler cone and the cone $\left\langle-e_{1},-\left(e_{1}+3 e_{2}\right)\right\rangle$ describes the $\mathrm{A}_{2}$ double point. This description of the $\mathrm{A}_{2}$ double point is related to that given e.g. in [32] by a change of basis $e_{1} \rightarrow-e_{2}, e_{2} \rightarrow-e_{1}+e_{2}$.

The general theory, due to Hironaka, tells us that the compactified moduli space can be resolved in such a way that all singularities of the Picard-Fuchs equations are regular divisors with normal crossings and part of the resolutions necessary to achieve this goal can be described by toric resolution if we start as above. Finding a resolution is necessary in order to analyse the full modular group. A thorough analysis of the modular groups for Calabi-Yau compactifications were given for one-moduli examples in $[11,13,14]$ and for two types of two-moduli examples in [15].

\footnotetext{
${ }^{8}$ This generalizes the reasoning, which leads for the quintic to the consideration of $z=1 / a^{5}[11]$ as the natural variable.

${ }^{9}$ Here the conventions are as in [32].
} 


\section{Local behaviour of the solutions, the mirror map and instanton corrected Yukawa couplings}

In this section we calculate the singular locus of the Picard-Fuchs equations and discuss some essential parts of the local behaviour of their solutions. We explain how to introduce canonical coordinates and fix a canonical form of the period vector. This leads to simple expressions of the instanton corrected Yukawa couplings and the Kähler potential in terms of the solutions. We will see that all topological data of the Calabi-Yau manifold appear naturally in this period vector.

It was shown in [33] that general Picard-Fuchs systems have only regular singular points, i.e. locally the solutions are given by power series or series involving finite powers of logarithms in $z_{i}$.

Let us now describe the singular locus (cf. [28]). Denote the linear partial differential operators of degree $m$, defined in a neighborhood $U$ of $z \in \mathscr{M}$ (the subspace of the complex structure moduli space to which our analysis applies), by $L_{i}=\sum_{|p| \leqslant m} a_{i}^{p}(z)(\mathrm{d} / \mathrm{d} z)^{p}$. They define a left ideal $I$ in the ring of partial differential operators on $U$. We now introduce the symbol of $L_{i}: \sigma\left(L_{i}\right)=$ $\Sigma_{|p|=m} a_{i}^{p}(z) \xi_{1}^{p_{1}} \cdots \xi_{k}^{p_{k}}$, where $\xi_{1}, \ldots, \xi_{k}$ is a coordinate system in the fiber of the cotangent bundle $T^{*} U$ at $z$. The ideal of symbols is defined by $\sigma(I)=\{\sigma(L) \mid L \in$ $I\}$. The singular locus is $S(I)=\pi(\mathrm{Ch}(I)-U \times\{0\})$, where the characteristic variety $\mathrm{Ch}(I)$ is the subvariety in $T^{*} U$ specified by the ideal of symbols and $\pi$ is the projection along the fiber of $T^{*} U$. The fact that $\sigma(I)$ is generated by $\sigma\left(L_{i}\right)$ is a special property of Picard-Fuchs systems. This follows e.g. from the way the Picard-Fuchs equations are derived by the Griffith-Dwork-Katz reduction method and simplifies the calculation of $S(I)$. Let us demonstrate this for $L_{1}, L_{2}$ given in Eq. (3.15). The symbols are

$$
\begin{aligned}
\sigma\left(L_{1}\right)= & z_{1}^{3}\left[\xi_{1}\left(1-27 z_{1}\right)-81 z_{2} \xi_{2}\right] \xi_{1}^{2}, \\
\sigma\left(L_{2}\right)= & z_{1}^{2}\left(1-27 z_{1}-27 z_{2}\right) \xi_{1}^{2}-3 z_{1} z_{2}\left(1+54 z_{2}\right) \xi_{1} \xi_{2} \\
& +9 z_{2}^{2}\left(1-27 z_{2}\right) \xi_{2}^{2} .
\end{aligned}
$$

Case by case analysis reveals that $\mathrm{Ch}(I)$ decomposes into the following components:

$$
\begin{aligned}
\operatorname{Ch}(I)= & \left\{81 z_{2} \xi_{2}-\left(1-27 z_{1}\right) \xi_{1}=\left(1-27 z_{1}\right)^{3}-27 z_{2}=0\right\} \cup\left\{z_{1}=z_{2}=0\right\} \\
& \cup\left\{\xi_{1}=\left(1-27 z_{2}\right)=0\right\} \cup\left\{\xi_{1}=z_{2}=0\right\} \cup\left\{\xi_{2}=z_{1}=0\right\} .
\end{aligned}
$$

Denoting the projections of the components on $U$ by $\Delta_{i}$ we have $S(I)=\prod_{i-0}^{3} \Delta_{i}$, with $\Delta_{0}=\left(1-27 z_{1}\right)^{3}-27 z_{2}, \Delta_{1}=1-27 z_{2}, \Delta_{2}=z_{1}, \Delta_{3}=z_{2}$.

The singularities $\Delta_{i}=0$ detected so far correspond to the discriminant locus in the moduli space on which the defining polynomials cease to be transverse, i.e. where $p_{1}=\ldots=p_{l}=0$ and $\mathrm{d} p_{1} \wedge \ldots \wedge \mathrm{d} p_{l}=0$ or, equivalently, where the Laurent polynomials fail to be $\Delta^{*}$ regular [26]. We calculate for comparison the discriminant of (3.12) at the end of Section 5.1. To study further singularities we have to compactify the moduli space. We can describe the compactification by the 
toric variety corresponding to the secondary fan constructed in the previous section. Then we find with the same method as above a singular locus of the Picard-Fuchs equation at the origin of the patch defined by $\sigma_{3}$, which is due to the $\mathbb{Z}_{9}$ identification of the parameter space discussed above. Such an analysis can be made for the general case and the singular locus determined this way is used in Section 7 in order to fix the holomorphic ambiguity for various examples.

From the definition of the (unnormalized) Yukawa couplings (coefficients of the cubic form $\Xi$ in [34]) and the variational property of the $(3,0)$ form [34] one finds (see also [35]) that the vanishing of the symbols at degree three gives relations for the Yukawa couplings by the simple replacement $\xi_{i} \xi_{j} \xi_{k} \rightarrow K_{z_{i} z_{j} z_{k}}$; e.g. from $\sigma\left(L_{1}\right)$ $=0$ one has $K_{z_{1} z_{1} z_{2}}=\left[\left(1-27 z_{1}\right) / 81 z_{2}\right] K_{z_{1} z_{1} z_{1}}$. This determines the Yukawa couplings up to a gauge dependent overall function. The gauge in which the PicardFuchs equation are derived is defined by (3.5), and the corresponding gauge dependent function can be calculated, up to a constant, by the methods outlined in $[11,16]$.

These unnormalized Yukawa couplings have singularities on the discriminant of the Calabi-Yau manifold. From the derivation of the Yukawa couplings in $[34,16]$ it follows that there is always one component which will appear in the denominator of all the Yukawa couplings, which we call the general component $\Delta_{0}$, following [36].

In the following we will demonstrate that the calculation of those Yukawa couplings on $\mathrm{X}^{*}$ which are functions of the complex structure moduli is not necessary for the purpose of determining the number of rational and elliptic curves. I.e. one can directly compute the instanton corrected couplings on X.

Because of the fact that the symbols of $I$ generate the ideal of symbols it is a rather simple algebraic problem to write down the associated first order pfaffian system, equivalent to the Gauss-Manin connection. However, we found that some local properties of the solutions are most easily obtained directly from $L_{1}, \ldots, L_{h}$. Most important is the analysis of the solutions around $z=0$, which is obviously a singular point in every system derived by factorization from (3.9). For the analysis it is useful to introduce the ring $\mathscr{R}$, which we define as the polynomial ring $\mathbb{C}[\theta]$ modulo the ideal $\mathscr{I}$ generated by the principal parts $I_{s}$ of the operators $L_{s}$,

$$
I_{s}(\theta)=\lim _{z \rightarrow 0} L_{s}(\theta, z),
$$

i.e. $\mathscr{R}=\mathbb{C}[\theta] / \mathscr{I}$. First we note that the $I_{s}(\theta)$ are homogeneous polynomials and the solution to $I_{s}=0 \forall s$ is $(2 k+2)$-times degenerate at $\theta=0$. $\mathbb{C}[\theta]$ has a natural vector space structure with the monomials as orthogonal basis. We can choose representatives of $\mathscr{R}$ as homogeneous polynomials orthogonal to $\mathscr{I}$. The subspace $\mathrm{R}$ of $\mathbb{C}[\theta]$ spanned by them has in all cases dimensions $\{1, k, k, 1\}$ at degrees $\{0,1,2,3\}$. As we will now show, it can be identified with the vector space of solutions to $L_{1}, \ldots, L_{h}$. The grading translates to the fact that we have one pure power series solution, $k$ solutions with a part linear in logarithms of $z, k$ solutions with a part quadratic in the logarithms of $z$ and one solution which has a part cubic in the logarithms. This identifies $z=0$ as a point where all but one cycle in $\mathrm{H}_{3}(\mathrm{X})$ are degenerate [37], which is also referred to as the point of maximally 
unipotent monodromy [38] and provides precisely the structure of solutions one needs for the mirror map.

Extending the definition of $x !=\Gamma(x+1)$ to $x \in \mathbb{R}$, we define the coefficient $c(n+\rho)$ for arbitrary values of the $k$ parameters $\rho_{i}$ and define the $\rho$-dependence of (3.7) as

$$
w_{0}(z, \rho)=\sum_{n_{i} \geqslant 0} c(n+\rho) z^{n+\rho} .
$$

By the method of Frobenius, the logarithmic solutions are obtained by taking linear combinations of derivatives $D_{\rho}=\Sigma\left(b_{n} / n !\right) \partial_{\rho}^{n}$ of $w_{0}(z, \rho)$ evaluated at $\rho=0$. Here we define $\partial_{\rho_{i}}:=(1 / 2 \pi i) \partial / \partial_{\rho_{i}}$. The factor $2 \pi i$ will make the monodromy matrix around $z=0$ integer. As $\left[L_{s}, \partial_{\rho_{i}}\right]=0$, it is then sufficient to check whether

$$
\left.D_{\rho}\left[L_{s} w_{0}(z, \rho)\right]\right|_{\rho=0}=0 \quad \forall s
$$

to establish $\left.D_{\rho} w_{0}(\mathrm{z}, \rho)\right|_{\rho=0}$ as a solution. By consideration of the explicit form of the series (4.4) one can show that the conditions for vanishing of the constant terms in (4.5),

$$
\left.D_{\rho}\left[I_{s}(\rho) c(0, \rho) z^{\rho}\right]\right|_{\rho=0}=0 \quad \forall s,
$$

are in fact also sufficient.

We introduce an identification of the ring $\mathbb{C}[\theta]$ with the ring of the partial derivatives with respect to (w.r.t.) $\rho$,

$$
\varphi: \quad \sum_{n_{i} \geqslant 0} b_{n} \theta^{n} \mapsto \sum_{n_{i} \leqslant 0} \frac{b_{n}}{n !} \partial_{\rho}^{n},
$$

which induces an isomorphism between the vector space $\mathrm{R}$ and the vector space of solutions to $L_{1}, \ldots, L_{k}$, as can be seen as follows. An element $r \in \mathrm{R}$ is orthogonal to all polynomials of the form $m(\theta) I_{s}$, where $m(\theta)$ is monomial in $\theta$. As $r$ and $I_{s}$ are homogeneous, one has to check orthogonality only for monomials $m(\theta)$ of degree $\operatorname{deg}(r)-\operatorname{deg}\left(I_{s}\right)$. Suppose $r$ is of lower degree, then $I_{s}$ (4.6) will obviously hold for $D_{\rho}=\varphi(r)$, because all terms in $D_{\rho}\left[I_{\mathrm{s}}(\rho) c(0, \rho) z^{\rho}\right]$ have positive degree in $\rho$ and will vanish, after setting $\rho$ to zero. If $\operatorname{deg}(r)=\operatorname{deg}\left(I_{s}\right)+n$, then $\operatorname{deg}(r)-n$ derivatives of $D_{\rho}=\varphi(r)$ have to act on $I_{s}$ to give a nonvanishing term. By our choice of the factorials the vanishing of these contributions is equivalent to $r \perp m(\theta) I_{s}=0$ for any $m(\theta)$ with $\operatorname{deg}(m(\theta))=n$. Hence $r \in \mathrm{R}$ iff $\left.\varphi(r) w_{0}(z, \rho)\right|_{\rho=0}$ is a solution.

Besides the unique power series solution (3.7) we choose for the $k$ solutions linear in the logarithms the basis $\left.\partial_{\rho_{i}} w(z, \rho)\right|_{\rho=0}$. They are given by

$$
w_{i}(z)=\sum_{n_{i} \leqslant 0} d_{i}(n) z^{n}+w_{0}(z) \frac{\log z_{i}}{2 \pi i},
$$

with

$$
d_{i}(n)=\left.\frac{1}{2 \pi i} \frac{\partial}{\partial \rho_{i}} c(n+\rho)\right|_{\rho=0} .
$$


The top element of $R$ is unique, up to a constant, which we will fix in a moment, and so is the solution cubic in the logarithms.

Defining now

$$
D_{i}^{(1)}:=\partial_{\rho_{i}}, \quad D_{i}^{(2)}:=\frac{1}{2} \tilde{K}_{i j k} \partial_{\rho j} \partial_{\rho k} \quad \text { and } \quad D^{(3)}:=-\frac{1}{6} \tilde{K}_{i j k} \partial_{\rho i} \partial_{\rho j} \partial_{\rho k},
$$

where summation is implicit, we have a natural basis for the period vector

$$
\Pi(z)=\left(\begin{array}{c}
w_{0}(z) \\
\left.D_{i}^{(1)} w_{0}(z, \rho)\right|_{\rho=0} \\
\left.D_{i}^{(2)} w_{0}(z, \rho)\right|_{\rho=0} \\
\left.D^{(3)} w_{0}(z, \rho)\right|_{\rho=0}
\end{array}\right)
$$

When one actually performs the derivatives w.r.t. $\rho_{i}$, one has to be careful when treating the poles of the Gamma-function and its derivatives. We clear up these technical details in Appendix A.

So far we have only dealt with the complex structure deformation parameters $z_{i}$. To describe the mirror map between the subsectors of the theory depending only on the complex structure parameters and the complexified Kähler structure parameters ${ }^{10}$, respectively, we will briefly review their common structure. They can be identified with topological field theories defined by the BRST operators $Q^{\mathrm{C}}=G^{+}+\bar{G}^{-}$and $Q^{\mathrm{K}}=G^{+}+\bar{G}^{+}$, respectively. The $G^{ \pm}, \bar{G}^{ \pm}$in $Q^{\mathrm{C}}$ and $Q^{\mathrm{K}}$ are related to the zero modes of the superpartners of the energy-momentum tensor of the underlying $N=2$ superconformal field theory by two different kinds of twist procedures which make either $Q^{\mathrm{C}}$ or $Q^{\mathrm{K}}$ to scalar operators [40,23]. At string tree level all relevant information is encoded in two prepotentials (free energy) $F^{\mathrm{C}}, F^{\mathrm{K}}$, which are sections of holomorphic line bundles $\mathrm{L}^{2}$ over the complex and Kähler structure moduli spaces, respectively. There exists a coordinate choice, the so-called inhomogeneous special coordinates $t^{i}$, such that the Yukawa couplings (structure constants) are ordinary third derivatives $\left(\partial_{i}=\partial / \partial t^{i}\right)$

$$
K_{i j k}=\partial_{i} \partial_{j} \partial_{k} F
$$

of the prepotential. For general coordinate choices the derivatives have to be covariantized w.r.t. the connection of the line bundle $\mathrm{L}$ and the metric on the moduli space. Unlike for e.g. topological models on manifolds with $c_{1}>0$ [41] the prepotential $F$ for Calabi-Yau threefolds cannot be derived from the associativity of the structure constants alone. For these cases one has on the other hand an additional geometrical structure known as special geometry, which was discovered first in the context of $N=2$ supergravity theories [42] and derived for the Kähler and complex structure moduli spaces of Calabi-Yau and/or $N=2$ string compact-

\footnotetext{
${ }^{10}$ Recall that the moduli describing the variation of the Riccic flat Kähler metric and the antisymmetric tensor background field can be parametrized by $\delta g_{j j} \mathrm{~d} z_{i} \wedge \mathrm{d} \bar{z}^{\bar{j}}=\sum_{i=1}^{k} \delta \hat{t}^{i} J_{i}$ and $\delta B_{i j} \mathrm{~d} z^{i} \wedge$ $\mathrm{d} \bar{z}^{j}=\sum_{i=1}^{k} \delta \tilde{t}^{i} J_{i}$, where one can combine $t^{i}=\tilde{t}^{i}+i \hat{t}^{i}$ to the so-called complexified Kähler structure parameter [39].
} 
ification in [43-45,39]. It implies that also the Kähler potential for the Weil-Peterson metric derives from the prepotential (hence the name) as

$$
K=-\log \left[\left(t^{i}-\bar{t}^{i}\right)\left(\partial_{i} F+\partial_{i} \bar{F}\right)+2 \bar{F}-2 F\right] .
$$

On the complex structure side the Yukawa couplings are expressed for a choice of the holomorphic threeform $\Omega$ as [34] $K_{i j k}=\int \Omega \partial_{i} \partial_{j} \partial_{k} \Omega$ and the Kähler metric is given by ${ }^{11} K=-\log \left(\Pi^{\dagger} \Sigma \Pi\right)$, where $\Pi$ is the period vector for a symplectic basis of $\mathrm{H}_{3}(\mathrm{X})$. As the holomorphic threeform $\Omega$ is defined only up to gauge transformations $\Omega \rightarrow f(z) \Omega(f(z)$ holomorphic) it takes values in a holomorphic line bundle $\mathrm{L}$ over the moduli space. The transformation properties of the quantities introduced are: $\Omega \in \mathrm{L}, F \in \mathrm{L}^{2}$, the Yukawas $K_{i j k}$ transform as components of

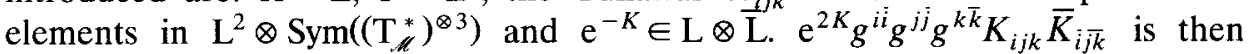
invariant under Kähler and coordinate transformations. The physical Yukawa couplings appearing in the effective lagrangian are those for canonically normalized matter fields.

To relate the solution of the Picard-Fuchs equations directly to $F$ we may use the form of the Picard-Fuchs differential operators in special coordinates in the Kähler gauge [46],

$$
\sum_{i=1}^{k} \partial_{j} \partial_{p}\left(K_{r}^{-1}\right)^{l i} \partial_{r} \partial_{i} \text {. }
$$

The period vector is then trivially expressed in terms of $F: \Pi(t)=\left(1, t^{i}, \partial_{i} F, 2 F-\right.$ $t^{i} \partial_{i} F$ ) (here and below we again use summation convention). The relation of the coordinates in Kähler gauge $t^{i}$ to the homogeneous special coordinates $\left(X^{0}, X^{i}\right)$, in which the period vector reads $\left(X^{0}, X^{i},\left(\partial \mathscr{F} / \partial X^{i}\right),\left(\partial \mathscr{F} / \partial X^{0}\right)\right)$, is given by $t^{i}=X^{i} / X^{0}$ with $\mathscr{F}=\left(X^{0}\right)^{2} F$.

Guided by the mirror hypothesis we should have the same structure for the Kähler side and therefore, for a formal large radius expansion of $F^{K}$,

$$
F^{K}=\frac{1}{6} K_{i j k}^{0} t^{i} t^{j} t^{k}+\frac{1}{2} a_{i j} t^{i} t^{j}+b_{i} t^{i}+\frac{1}{2} c+F_{\text {inst. }}
$$

around $\operatorname{Im}\left(t^{i}\right) \rightarrow \infty$, the "period vector"

$$
X^{0}\left(\begin{array}{c}
1 \\
t^{i} \\
\frac{1}{2} K_{i j k}^{0} t^{j} t^{k}+a_{i j} t^{j}+b_{i}+\partial_{t_{i}} F_{\text {inst. }} \\
-\frac{1}{6} K_{i j k}^{0} t^{i} t^{j} t^{k}+b_{i} t^{i}+c+\left(2 F_{\text {inst. }}-t^{i} \partial_{t_{i}} F_{\text {inst. }}\right)
\end{array}\right)=X^{0} \Pi(t) .
$$

Here the $K_{i j k}^{0}$ are the classical intersections calculated in Section 2 and $F_{\text {inst }}$. is a power series in $q_{i}=\exp \left(2 \pi i t^{i}\right)$, which encodes the instanton corrections.

Starting from $(4.10)$ in $z_{i}$ coordinates we now have to make a coordinate

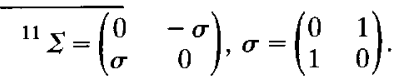


transformation to suitable inhomogenous coordinates, which are defined by ratios of solutions of the Picard-Fuchs equations as

$$
t^{i}(z)=\frac{w_{i}(z)}{w_{0}(z)} .
$$

The reason for picking this quotient is that the invariance of the theory under the monodromies around $z_{i}=0$ can be identified with the invariance of the topological sigma model under real shifts $t^{i} \rightarrow t^{i}+1$ of the antisymmetric background field or equivalently of the effective field theory under discrete Peccei-Quinn symmetries. As will become clear below, this choice identifies the form of the prepotential for the complex structure moduli space on $\mathrm{X}^{*}$ with the one expected for the Kähler structure moduli space on X. It strongly resembles the elliptic curve case where the ratios of two arbitrary solutions of the period equation and its inverse satisfy a third order non-linear differential equation introduced by Schwarz. Inserting the solutions $z(t)$ in the $J$ invariant of the elliptic curve gives the well-known expansion $J((a t+b) /(c t+d))=\frac{1}{1728}\left(q^{-2}+144+\ldots\right)$ of the $J$-function in terms of $q=\exp [2 \pi i(a t+b) /(c t+d)]$. The choice of the logarithmic solution for $w_{1}$ and the power series solution for $w_{0}$ at the point of maximal unipotent monodromy then corresponds to $a=d=1, b=c=0$. For Calabi-Yau spaces one finds likewise that the mirror maps $z_{i}\left(q_{1}, \ldots, q_{h}\right)$ always have an integral expansion, which is a necessary condition for obtaining integer instanton numbers. One therefore expects that the mirror map plays an important rôle for the theory of modular forms on the moduli space of Calabi-Yau space, which, however, seems to be much more intricate, as the modular group acts in general non-arithmetically on the geometrical parameters.

Inserting the inverse map of (4.16) into (4.10) we obtain the transformed period vector

$$
\tilde{\Pi}(t)=w_{0}(t)\left(\begin{array}{c}
1 \\
t^{i} \\
\frac{1}{2} \tilde{K}_{i j k}^{0} t^{j} t^{k}+\hat{b}_{i}+\partial_{t_{i}} \tilde{F}_{\text {inst. }} \\
-\frac{1}{6} \tilde{K}_{i j k}^{0} t^{i} t^{j} t^{k}-\hat{b}_{i} t^{i}+\hat{c}+\left(2 \tilde{F}_{\text {inst. }}-t^{i} \partial_{t_{i}} \tilde{F}_{\text {inst. }}\right)
\end{array}\right) .
$$

By comparison with (4.15) we can view all quantities marked with a tilde, up to one overall normalization, as predictions of mirror symmetry. Especially we have to identify $K_{i j k}^{0}$ with $\tilde{K}_{i j k}^{0}$. In fact, for all complete intersection cases the top element of the ring $\mathrm{R}$ encodes the intersection numbers in the integral basis of divisors coming from the ambient spaces. This turns out to be true also for the hypersurfaces discussed in [16], for the basis of divisors which generate $\mathrm{H}_{2}(X, \mathbb{Z})$.

After fixing the normalization, we see that the instanton corrected Yukawa coupling $K_{i j k}$ can be uniquely expressed by the solutions of the Picard-Fuchs equations as

$$
K_{i j k}(t)=\partial_{t_{i}} \partial_{t_{j}} \frac{\left.D_{k}^{(2)} w_{0}\right|_{\rho=0}}{w_{0}}(t)
$$


or, equivalently, by the derivatives of the prepotential

$$
F(t)=\left.\frac{1}{2}\left(\frac{1}{w_{0}}\right)^{2}\left(w_{0} D^{(3)} w_{0}+D_{l}^{(1)} w_{0} D_{l}^{(2)} w_{0}(t)\right)\right|_{\rho=0} .
$$

We will use formulas (4.18), (4.19), which do not require the evaluation of the Yukawa couplings on the mirror manifold, to compute a convergent expansion for the instanton corrected Yukawas and the prepotential in the large radius limit and to predict the number of rational curves for various manifolds.

So far we have seen that the natural choice for the period vector (4.10) matches the leading terms in $t$ of the components of (4.15) and leads to a prediction of the instanton corrections. Let us now compute also the lower order terms in $t$, i.e. the constants $\hat{b}_{i}, \hat{c}$. For this purpose one has to take the derivatives w.r.t. $\rho$ explicitly,

$$
\begin{aligned}
& \frac{\partial}{\partial \rho_{i}} c(0)=-\left(\sum_{\alpha} l_{0 \alpha}^{(i)}+\sum_{\alpha} l_{\alpha}^{(i)}\right) \Gamma^{\prime}(1)=: c_{1}^{i} \Gamma^{\prime}(1)=0, \\
& \frac{\partial}{\partial \rho_{i}} \frac{\partial}{\partial \rho_{j}} c(0)=\frac{\pi^{2}}{6}\left(\sum_{\alpha} l_{0 \alpha}^{(i)} l_{0 \alpha}^{(j)}-\sum_{\alpha} l_{\alpha}^{(i)} l_{\alpha}^{(j)}\right)=: \frac{\pi^{2}}{3} c_{2}^{i j}, \\
& \frac{\partial}{\partial \rho_{i}} \frac{\partial}{\partial \rho_{j}} \frac{\partial}{\partial \rho_{k}} c(0)=2\left(\sum_{\alpha} l_{0 \alpha}^{(i)} l_{0 \alpha}^{(j)} l_{0 \alpha}^{(k)}+\sum_{\alpha} l_{\alpha}^{(i)} l_{\alpha}^{(j)} l_{\alpha}^{(k)}\right) \zeta(3)=: 6 c_{3}^{i j k} \zeta(3),
\end{aligned}
$$

where the coefficients $c_{n}$ coincide for complete intersections in products of nonsingular weighted projective spaces with the ones given in (2.4). Using the Gauss-Bonnet formula we get from this the following remarkable identities:

$$
\begin{aligned}
& \int_{\mathrm{X}} c_{2} J_{i}=\int_{\mathrm{X}} c_{2}^{j k} J_{j} J_{k} J_{i}=\frac{3}{\pi^{2}} \int_{\mathrm{X}} J_{j} J_{k} J_{i} \frac{\partial}{\partial \rho_{j}} \frac{\partial}{\partial \rho_{k}} c(0)=-24 D_{i}^{(2)} c(0), \\
& \chi=\int_{\mathrm{X}} c_{3}^{i j k} J_{i} J_{j} J_{k}=\frac{1}{6 \zeta(3)} \int_{\mathrm{X}} J_{i} J_{j} J_{k} \frac{\partial}{\partial \rho_{i}} \frac{\partial}{\partial \rho_{j}} \frac{\partial}{\partial \rho_{k}} c(0)=i \frac{(2 \pi)^{3}}{\zeta(3)} D^{(3)} c(0),
\end{aligned}
$$

with $D_{i}^{(2)}$ and $D^{(3)}$ defined in (4.9), which express the Euler number and $\int_{\mathrm{X}} c_{2} J_{i}$ in terms of the intersection numbers and the generators of the Mori cone $l^{(i)}$. These identities hold also for the canonically resolved manifolds, if the complete intersection had canonical quotient singularities. The linear terms in the third row of (4.17) are thus $\hat{b}_{i}=-\frac{1}{24} \int_{M} c_{2} J_{i}$ and in the last row $\hat{c}=-i \zeta(3)_{\chi} /(2 \pi)^{3}$.

The imaginary part of the constant $\hat{c}$ can be identified with a $\sigma$-model loop contribution and is proportional to the Euler number of the manifold. The constant of proportionality seems to be universal and has been calculated explicitly for the quintic hypersurface in $\mathbb{P}^{4}$ and other one-moduli cases $[14,13]$ as $-i \zeta(3) /(2 \pi)^{3} \cdot \operatorname{Im}\left(a_{i j}=\operatorname{Im}\left(b_{i}\right)=0\right.$ is a necessary condition for having a continuous Peccei-Quinn symmetry $t_{j} \rightarrow t_{j}+\alpha_{j}$, $\alpha_{j}$ real, which is broken by instanton corrections to discrete shifts $t^{i} \rightarrow t^{i}+1$ as mentioned before.

While the real constants $b_{i}, a_{i j}$ in (4.15) are irrelevant for physical quantities, they are important for fixing an integral symplectic basis for the period vector. In 
fact we find that the constant $\hat{b}_{i}$ for the one-moduli cases $[13,14]$ is also correctly reproduced in the third line. The constants in front of the first subleading terms in (4.17) do not correspond to a choice of an integral basis as can be seen by comparison with (4.13). Especially the constants $a_{i j}$ do not seem to be directly related to topological numbers; in fact there are none further at our disposal. They have to be fixed by analysing the monodromy matrices themselves. The monodromy operation $T_{i}: t^{i} \rightarrow t^{i}+1$ on (4.15) is obvious. The requirement that it is integral and symplectic yields restrictions on $a_{i j}$, e.g. for the one-moduli cases $a=\left(\frac{1}{2} \mathrm{~K}^{0} \bmod \mathbb{Z}\right)\left(\right.$ and $\left.2 b=\left(\left(1-\frac{1}{6} \mathrm{~K}^{0}\right) \bmod \mathbb{Z}\right)\right)$. The integer part is irrelevant as it can be absorbed by an $\mathrm{SP}(4, \mathbb{Z})$ transformation, hence the basis can be specified. Although we have no general proof, it is tempting to conjecture that the occurence of the topological numbers $K_{i j k}^{0}, \chi$ and, up to SP $(2 h+2, \mathbb{Z})$ transformations, also $\int_{\mathrm{X}} c_{2} J_{i}$ in $\mathrm{F}$ at the point of maximal unipotent monodromy in a integral sympletic basis is a general feature.

The instanton part of the Yukawa couplings comes from stationary points of the classical string action, which correspond to holomorphic mappings from $\mathbb{P}^{1}$ to the CY manifold. In the coordinates $t^{i}$ defined in (4.16) it enjoys the following expansion:

$$
\begin{aligned}
K_{k l m}= & \int_{\mathrm{X}} J_{k} \wedge J_{l} \wedge J_{m}+\sum_{d} \int_{\mathscr{M}_{C_{d}}} \mathrm{~d} \mu \exp \left(2 \pi i \int_{\mathrm{C}_{d}} K(X)\right) \\
& \times\left[1-\exp \left(2 \pi i \int_{\mathrm{C}_{d}} K(X)\right)\right]^{-1} \\
= & K_{k l m}^{0}+\sum_{d_{1}, \ldots, d_{k}} \frac{n_{d_{1}, \ldots, d_{k}}^{r} d_{k} d_{l} d_{m}}{1-\prod_{i=1}^{k} q_{i}^{d_{i}}} \prod_{i=1}^{k} q_{i}^{d_{i}} .
\end{aligned}
$$

Here we have defined the degree of the curve as $d_{i}=\int_{\mathrm{C}} J_{i}$, which is an integer for a basis $J_{i} \in \mathrm{H}^{1,1}(\hat{\mathrm{X}}, \mathbb{Z})$. The denominator $\left(1-\exp \left[\int_{C_{d}} K(X)\right]\right)$ gives the correct combinatorical contributions from multi-covers of the curves so that the integral $\mathrm{d} \mu$ of the Euler class over the compactified moduli space $\mathscr{M}_{\mathrm{C}_{d}}$ of holomorphic maps of multi-degree $d$ from $\mathbb{P}^{1}$ [47] can be taken over single cover curves only. The resulting invariants $n_{d_{1}, \ldots, d_{k}}^{\mathrm{r}}$ are expected to be integers, for isolated curves they just count their numbers. In [16], examples of negative invariants $n^{\mathrm{r}}$ were found. They admit only the interpretation that there are non-isolated singular curves at the corresponding degree. The occurence of the terms $d_{k} d_{l} d_{m}$ in the third line is due to the integral of the part of the moduli space describing the reparametrization of $\mathbb{P}^{1}$, as was explained for isolated curves in [48].

\section{Selected examples}

1. As our first example we will calculate the topological invariants $n^{\mathrm{r}}$ for the manifold (3.10). The ideal $\mathscr{I}$ is generated by $I_{1}=\theta_{1}^{2}-3 \theta_{1} \theta_{2}+9 \theta_{2}^{2}$ and $I_{2}=\theta_{1}^{3}$, so 
Table 1

\begin{tabular}{lrlr}
\hline$\left(d_{J_{1}}, d_{J_{2}}\right)$ & $n^{\mathrm{r}}$ & $\left(d_{J_{1}}, d_{J_{2}}\right)$ & \multicolumn{1}{c}{$n^{\mathrm{r}}$} \\
\hline$(1,0)$ & 243 & $(1,1)$ & 972 \\
$(2,0)$ & 243 & $(2,2)$ & 156249 \\
$(3,0)$ & 54 & $(3,3)$ & 60018786 \\
$(4,0)$ & 243 & $(2,1)$ & 15309 \\
$(5,0)$ & 243 & $(4,2)$ & 111401163 \\
$(6,0)$ & 54 & $(3,1)$ & 179901 \\
$(0,1)$ & 63 & $(4,1)$ & 1558845 \\
$(0,2)$ & 63 & $(5,1)$ & 11558295 \\
$(0,3)$ & 54 & $(1,2)$ & 3402 \\
$(0,4)$ & 63 & $(2,4)$ & 4803867 \\
$(0,5)$ & 63 & $(3,2)$ & 4830597 \\
$(0,6)$ & 54 & $(1,3)$ & 9720 \\
& & $(2,3)$ & 977589 \\
& & $(1,4)$ & 25515 \\
& & $(1,5)$ & 61236 \\
\hline
\end{tabular}

$\mathrm{R}$ is spanned by

$$
\begin{gathered}
1, \\
\theta_{1}, \quad \theta_{2}, \\
9 \theta_{1} \theta_{2}+3 \theta_{2}^{2}, \quad 9 \theta_{1}^{2}+3 \theta_{1} \theta_{2}, \\
9 \theta_{1}^{2} \theta_{2}+3 \theta_{1} \theta_{2}^{2} .
\end{gathered}
$$

By (4.7) this translates to a basis of solutions for $L_{1}, L_{2}$ as one may verify. The fact that the top element of $\mathrm{R}$ coincides, up to scaling, with $K^{0}=9 J_{1}^{2} J_{2}+3 J_{1} J_{2}^{2}$, calculated by (2.5), is a nontrivial check on the mirror hypothesis. Using (4.18) one obtains concise formulas for the instanton corrected intersection numbers,

$$
\begin{aligned}
& K_{i, j, 1}(t)=\partial_{t_{i}} \partial_{t_{j}} \frac{\left.\left(9 \partial_{\rho_{1}} \partial_{\rho_{2}}+\frac{3}{2} \partial_{\rho_{2}}^{2}\right) w_{0}\right|_{\rho=0}}{w_{0}}(t), \\
& K_{i, j, 2}(t)=\partial_{t_{i}} \partial_{t_{j}} \frac{\left.\left(\frac{9}{2} \partial_{\rho_{1}}^{2}+3 \partial_{\rho_{1}} \partial_{\rho_{2}}\right) w_{0}\right|_{\rho=0}}{w_{0}}(t),
\end{aligned}
$$

from which the topological invariants $n^{\mathrm{r}}$ follow by comparison with (4.22). In Table 1 we display them for multi-degrees $\left(d_{J_{1}}, d_{J_{2}}\right), d_{J_{1}}+d_{J_{2}} \leqslant 6$. In fact, $J_{i}$ is a basis of a subspace of $\mathrm{H}^{2}(\mathrm{X}, \mathbb{Z})$ in which the Kähler cone is simply given by

$$
\sigma(K)=\left\{\sum_{i} t_{i} J_{i} \mid t_{i}>0\right\}
$$


In [10] a three-generation model $(\chi=-6)$ was constructed, by dividing the manifold (3.10) by a $G=\mathbb{Z}_{3} \times \mathbb{Z}_{3}$ symmetry and resolving the singular quotient. The group $\mathrm{G}$ is generated, on the homogeneous coordinates of $\mathbb{P}^{3} \times \mathbb{P}^{2}$, by $\left(x_{0}, x_{1}\right.$, $\left.x_{2}, x_{3}, y_{1}, y_{2}, y_{3}\right) \rightarrow\left(x_{0}, x_{1}, x_{2}, x_{3}, \alpha y_{1}, y_{2}, \alpha^{-1} y_{3}\right)\left(\alpha=\mathrm{e}^{2 \pi i / 3}\right)$, with three fixed tori $\mathrm{T}_{i}=\left\{x \in \mathbb{P}^{3} \mid x_{i}=0, p_{1}=0\right\}, i=1,2,3$, and a freely acting cyclic permutation $\left(x_{0}, x_{1}, x_{2}, x_{3}, y_{1}, y_{2}, y_{3}\right) \mapsto\left(x_{0}, x_{2}, x_{3}, x_{1}, y_{2}, y_{3}, y_{1}\right)$. This action is only compatible with a subspace of the moduli space of complex structure deformations, namely with the following perturbations:

$$
\begin{aligned}
& p_{1}=\sum_{i=1}^{3} x_{i} y_{i}^{3}-\tilde{a}_{1} x_{0} y_{1} y_{2} y_{3}, \\
& p_{2}=\sum_{i=0}^{3} x_{i}^{3}-\tilde{a}_{2} x_{1} x_{2} x_{3} .
\end{aligned}
$$

To relate this description to (3.4), one relates the variables $X_{1, i}(i=1, \ldots, 4)$ and $X_{2, j}(j=1,2,3)$ in (3.4) to the following Laurent monomials of the homogeneous coordinates of $\mathbb{P}^{3} \times \mathbb{P}^{2}$ :

$$
\begin{aligned}
& X_{1,1}=\frac{y_{1}^{3} x_{1}}{x_{0} y_{1} y_{2} y_{3}}, \quad X_{1,2}=\frac{y_{2}^{3} x_{2}}{x_{0} y_{1} y_{2} y_{3}}, \quad X_{1,3}=\frac{y_{3}^{3} x_{3}}{x_{0} y_{1} y_{2} y_{3}}, \quad X_{1,4}=\frac{x_{0}^{3}}{x_{1} x_{2} x_{3}}, \\
& X_{2,1}=\frac{x_{1}^{3}}{x_{1} x_{2} x_{3}}, \quad X_{2,2}=\frac{x_{2}^{3}}{x_{1} x_{2} x_{3}}, \quad X_{2,3}=\frac{x_{1}^{3}}{x_{1} x_{2} x_{3}},
\end{aligned}
$$

by which we can identify (3.12) with (5.4) and the parameter $a_{i}$ with $\tilde{a}_{i}\left(a_{i j}=1\right)$. Furthermore we see that (3.12) is invariant under $G$, which implies that the subsystem of the moduli space we are considering is in the invariant sector of $G$ and part of the moduli space of the three-generation model.

The mirror manifold can also be constructed as the quotient of (5.4) w.r.t. the group of order 27 generated by $\left(x_{0}, x_{1}, x_{2}, x_{3}, y_{1}, y_{2}, y_{3} ; a_{1}, a_{2}\right) \mapsto\left(x_{0}\right.$, $\left.\beta^{3(m+n)} x_{1}, \beta^{6 m} x_{2}, \beta^{6 n} x_{3}, \beta^{-m-n} y_{1}, \beta^{-8 m} y_{2}, \beta^{-8 n} y_{3} ; a_{1}, a_{2}\right)$ with $\beta=\mathrm{e}^{2 \pi i / 9}, m$, $n \in \mathbb{Z}$. The full invariance group of (5.4) is generated by the above transformations and $\left(x_{0}, x_{1}, x_{2}, x_{3}, y_{1}, y_{2}, y_{3} ; a_{1}, a_{2}\right) \mapsto\left(x_{0}, \beta^{3 k} x_{1}, x_{2}, x_{3}, \beta^{-k} y_{1}, y_{2}, y_{3} ; \beta^{k} a_{1}\right.$, $\left.\beta^{6 k} a_{2}\right), k \in \mathbb{Z}$, i.e. we have the same identification of the parameter space of the mirror manifold as we found for (3.12).

We can always find a map analogous to (5.5) which maps the Laurent polynomials $P_{1}, \ldots, P_{l}$ to quasi-homogeneous polynomials $p_{1}, \ldots, p_{l}$. In terms of those the fundamental period $w_{0}$ can be obtained as the integral [17]

$$
w_{0}=\prod_{i=1}^{l}\left(-a_{i}\right) \int \frac{1}{p_{1} \cdots p_{l}} \mathrm{~d} x_{1,1} \cdots \mathrm{d} x_{k, n_{k}+1}
$$

with integration contours $\left|x_{i, j}\right|=1$. While we can always define a map such that $w_{0}$ is given by this expression, in most of the cases, however, this map cannot be chosen such that the polynomials $p_{1}, \ldots, p_{l}$ define a transverse configuration in 
$\mathbb{P}^{n_{1}}\left[\boldsymbol{w}^{(1)}\right] \times \cdots \times \mathbb{P}^{n_{k}}\left[\boldsymbol{w}^{(k)}\right]$. An example of such a case will be given at the end of Section 6 .

Let us demonstrate the direct calculation of the discriminant locus for the manifold defined in (3.12) by examining the conditions of $\Delta$-regularity which reads that for all faces $\Theta_{1}^{*}$ and $\Theta_{2}^{*}$ of $\Delta_{1}$ and $\Delta_{2}^{*}$ we need $P_{\Theta_{1}^{*}}=P_{\Theta_{2}^{*}}=0$ and that all $2 \times 2$ subdeterminants of the matrix whose two rows are $X_{i, j} \partial P_{\Theta_{i}^{*}} / \partial X_{i, j}$ and $X_{i, j} \partial P_{\Theta_{2}^{+}} / \partial X_{i, j}=0$ (no sum) vanish simultaneously.

For convenience we rename the parameters $a_{i}, a_{i, j}$ occuring in $\mathrm{P}_{1}$ and $\mathrm{P}_{2}$ as $a_{0}, \ldots, a_{4}$ and $b_{0}, \ldots, b_{3}$. If we introduce variables $U_{i} \equiv U_{1, i}$ and $V_{j} \equiv U_{2, j}$ such that $X_{i, j}=U_{i, j} / U_{i, 0}$ the conditions for non-regularity become for $\Theta_{1}^{*}=\Delta_{1}^{*}$ and $\Theta_{2}^{*}=\Delta_{2}^{*}$,

$$
\begin{aligned}
& \sum_{i=0}^{3} a_{i} U_{i}=0, \quad \sum_{j=0}^{3} b_{j} V_{j}+a_{4} U_{4}=0, \\
& \frac{a_{4} U_{4}}{V_{0} U_{0}}\left(a_{i} U_{i}-a_{j} U_{j}\right)=0, \quad \frac{a_{i} U_{i}}{U_{0} V_{0}}\left(b_{3} V_{3}-b_{k} V_{k}\right)=0, \\
& i, j=1,2,3, \quad k=1,2 .
\end{aligned}
$$

Note that $U_{1} U_{2} U_{3} U_{4} / U_{0}^{3} V_{0}=V_{1} V_{2} V_{3} / V_{0}^{3}=1$. There are several ways to satisfy the second set of equations. E.g. if we choose to satisfy them by equating the expressions in parantheses to zero, we find, using the variables $z_{1}=a_{1} a_{2} a_{3} a_{4} / a_{0}^{3} b_{0}$, $z_{2}=b_{1} b_{2} b_{3} / b_{0}^{3}$ and introducing $A=a_{4} U_{4}$ and $B=b_{0} V_{0}$, that $z_{1}=-\frac{1}{27} A / B$ and $z_{2}=\frac{1}{27}(A+B)^{3} / B^{3}$, which satisfy $\left(1-27 z_{1}\right)^{3}-27 z_{2}=0$. Another possible choice is to set $a_{4} U_{4}=0$ and $b_{3} V_{3}-b_{\mathrm{k}} V_{\mathrm{k}}=0$. This leads to $1-27 z_{2}=0$. Other components of the discriminant can be obtained by considering other (lower dimensional) faces of $\Delta_{1}^{*}$ and $\Delta_{2}^{*}$.

2. Our next example is the Tian-Yau manifold given by the configuration

$$
\left(\begin{array}{c||ccc}
\mathbb{P}^{3} & 3 & 0 & 1 \\
\mathbb{P}^{3} & 0 & 3 & 1
\end{array}\right)_{-18}^{14}: \begin{gathered}
K^{0}=9 J_{1}^{2} J_{2}+9 J_{1} J_{2}^{2} \\
c_{2} J_{1}=c_{2} J_{2}=36
\end{gathered}
$$

The quotient of this manifold w.r.t. the $\mathbb{Z}_{3}$ group acting by $\left(x_{0}, x_{1}, x_{2}, x_{3}, y_{0}, y_{1}\right.$, $\left.y_{2}, y_{3}\right) \mapsto\left(x_{0}, x_{1}, x_{2}, \alpha x_{3}, y_{0}, y_{1}, y_{2}, \alpha^{2} y_{3}\right)\left(\alpha=\mathrm{e}^{2 \pi i / 3}\right)$ on the homogeneous coordinates of $\mathbb{P}^{3} \times \mathbb{P}^{3}$ yields a simple realisation of a three-generation compactification, which is diffeomorphically equivalent to the one discussed in Section 5.1. A preliminary phenomenological discussion was given in $[49,50]$. For the above configuration all complex structure deformations can be represented as monomials. Hence the full moduli dependence of all Yukawa couplings and the Kähler potential for 27's and $\overline{27}$ 's can in principle be calculated by a straightforward, though very tedious, application of the methods described in $[16,15]$.

The vertices of the dual polyhedra are now $\nu_{1,1}^{*}=(1,0,0 ; 0,0,0), \ldots, \nu_{2,4}^{*}=(0$, $0,0 ;-1,-1,-1)$ which we group into three sets as $E_{1}=\left\{\nu_{1,1}^{*}, \nu_{1,2}^{*}, \nu_{1,3}^{*}\right\}$,

\footnotetext{
${ }^{12}$ The results are of course again independent of the way we group the vertices into three sets, as long as the first and second sets contain three vertices pertaining to the first and the second $\mathbb{P}^{3}$, respectively.
} 
$\mathrm{E}_{2}=\left\{\nu_{2,1}^{*}, \nu_{2,2}^{*}, \nu_{2,3}^{*}\right\}$ and $\mathrm{E}_{3}=\left\{\nu_{1,4}^{*}, \nu_{2,4}^{*}\right\}^{12}$. This corresponds to the three Laurent polynomials

$$
\begin{aligned}
& P_{1}=a_{0}-a_{1} X_{1}-a_{2} X_{2}-a_{3} X_{3}, \quad P_{2}=b_{0}-b_{1} Y_{1}-b_{2} Y_{2}-b_{3} Y_{3}, \\
& P_{3}=c_{0}-\frac{c_{1}}{X_{1} X_{2} X_{3}}-\frac{c_{2}}{Y_{1} Y_{2} Y_{3}} .
\end{aligned}
$$

The period (3.7) follows straightforwardly by performing the integral (see (3.5))

$$
w_{o}\left(z_{1}, z_{2}\right)=\int_{\left|X_{i}\right|=\left|Y_{j}\right|=1} \frac{a_{0} b_{0} c_{0}}{P_{1} P_{2} P_{3}} \Pi \frac{\mathrm{d} Y_{i}}{Y_{i}} \frac{\mathrm{d} X_{i}}{X_{i}},
$$

which gives $w_{0}\left(z_{1}, z_{2}\right)$ as in Eq. (3.7) with $z_{1}=a_{1} a_{2} a_{3} c_{1} / a_{0}^{3} c_{0}$ and $z_{2}=$ $b_{1} b_{2} b_{3} c_{2} / b_{0}^{3} c_{0}$. This corresponds to introducing extended vertices $\bar{\nu}^{*}=\left(\overrightarrow{\mathrm{e}}_{i}, \nu^{*}\right)$, where $\overrightarrow{\mathrm{e}}_{1}=(1,0,0), \overrightarrow{\mathrm{e}}_{2}=(0,1,0)$ and $\overrightarrow{\mathrm{e}}_{3}=(0,0,1)$ for the vertices in the three sets. The linear relations between the $\bar{\nu}^{*}$ are then in accordance with the general formula (3.9) and read

$$
\begin{aligned}
& l^{(1)}=(-3,0,-1 ; 1,1,1,1,0,0,0,0), \\
& l^{(2)}=(0,-3,-1 ; 0,0,0,0,1,1,1,1) .
\end{aligned}
$$

The associated differential operators

$$
\begin{aligned}
& \mathscr{L}_{1}=\theta_{1}^{3}-3\left(\theta_{1}+\theta_{2}\right)\left(3 \theta_{1}-1\right)\left(3 \theta_{1}-2\right) z_{1}, \\
& \mathscr{L}_{2}=\theta_{2}^{3}-3\left(\theta_{1}+\theta_{2}\right)\left(3 \theta_{2}-1\right)\left(3 \theta_{2}-2\right) z_{2}
\end{aligned}
$$

can be factorized, $\mathscr{L}_{1}+\mathscr{L}_{2} \equiv\left(\theta_{1}+\theta_{2}\right) L_{2}$, with the second order operator $\left(L_{1}=\mathscr{L}_{1}\right)$

$$
L_{2}=\left(\theta_{1}^{2}-\theta_{1} \theta_{2}+\theta_{2}^{2}\right)-3\left(3 \theta_{1}-1\right)\left(3 \theta_{1}-2\right) u-3\left(3 \theta_{2}-1\right)\left(3 \theta_{2}-2\right) z_{2} .
$$

The Yakawa couplings are

$$
\tilde{K}^{(3,0)}=\frac{1}{z_{2}^{2} \Delta_{0} \Delta_{1}^{2}}, \quad \tilde{K}^{(2,1)}=\frac{1}{27 z_{1}^{2} z_{2} \Delta_{0} \Delta_{1}},
$$

where the general component of the discriminant surface is $\Delta_{0}=1-27 z_{1}-27 z_{2}$ and a second component reads $\Delta_{1}=1-27 z_{1}$. For symmetry reasons $\tilde{K}^{(0,3)}, K^{(1,2)}$ are given by the above expressions but with $z_{1}$ and $z_{2}$ exchanged. Due to this symmetry we list in Table 2 the invariants $n_{d_{1}, d_{2}}^{\mathrm{r}}$ only for $d_{1} \leqslant d_{2}$.

Table 2

\begin{tabular}{lllrll}
\hline$\left(d_{1}, d_{2}\right)$ & $n_{\mathrm{d}_{1}, \mathrm{~d}_{2}}^{\mathrm{r}}$ & $\left(d_{1}, d_{2}\right)$ & $n_{\mathrm{d}_{1}, \mathrm{~d}_{2}}^{\mathrm{r}}$ & $\left(d_{1}, d_{2}\right)$ & $n_{\mathrm{d}_{1}, \mathrm{~d}_{2}}^{\mathrm{r}}$ \\
\hline$(0,1)$ & 81 & $(1,1)$ & 729 & $(1,3)$ & 6885 \\
$(0,2)$ & 81 & $(2,2)$ & 33534 & & \\
$(0,3)$ & 18 & $(3,3)$ & 5433399 & $(1,4)$ & 18954 \\
$(0,4)$ & 81 & $(1,2)$ & 2187 & $(1,5)$ & 45927 \\
$(0,5)$ & 81 & $(2,4)$ & 1708047 & $(2,3)$ & 300348
\end{tabular}


The number of curves up to degree three agree with those calculated by algebraic counting methods in [21]. This calculation confirms the lines $(0,1)$ and the degree $(0,2),(2,1)$ curves on this CICY. According to [21] there are no $(0,3)$ curves and 567 degree $(1,1)$ curves, leaving aside the possibility of nodal cubics and degenerate rational curves, respectively. More recently, all entries up to degree three have been confirmed [51]. The invariant $n_{0,3}^{r}=18$ was calculated as the Euler number of the tangent bundle of the moduli space of a family of nodal cubics.

In Appendix B we present general formulas for the predicted numbers of lines for all possible manifolds of the type of Section 5.1 in $\mathbb{P}^{3} \times \mathbb{P}^{2}$ and of Section 5.3 in $\mathbb{P}^{3} \times \mathbb{P}^{3}$. In deriving these formulas, we utilized formula (4.18) for the instanton corrected Yukawa couplings.

Next we will treat two examples, which involve a somewhat more complicated factorization procedure, but skip in the following most of the details.

3 . For the manifold defined by the configuration matrix

$$
\left(\begin{array}{l||lll}
\mathbb{P}^{3} & 3 & 1 & 0 \\
\mathbb{P}^{3} & 1 & 1 & 2
\end{array}\right)_{-120}^{12}
$$

the linear differential operators (3.9) are, after trivial factorization, of fourth and third order, respectively. We get a second order operator by factorising $27 \mathscr{L}_{1}+$ $\left(40 \theta_{1}+13 \theta_{2}\right) \mathscr{L}_{2}=\left(\theta_{1}+\theta_{2}\right)\left(3 \theta_{1}+\theta_{2}\right) L_{1}$, with

$$
\begin{aligned}
L_{1}= & \left(9 \theta_{1}-12 \theta_{1} \theta_{2}+13 \theta_{2}^{2}\right)-27\left(3 \theta_{1}+\theta_{2}-1\right)\left(3 \theta_{1}+\theta_{2}-2\right) z_{1} \\
& -2\left(2 \theta_{2}-1\right)\left(40 \theta_{1}+13 \theta_{2}\right) z_{2} .
\end{aligned}
$$

Due to their complexity, we refrain from giving the Yukawa couplings explicitly. The expansion of the prepotential by (4.19) does not require their knowledge. From the first few terms in the expansion of the prepotential,

$$
\begin{aligned}
F= & \frac{2}{6}\left(t^{1}\right)^{3}+\frac{6}{2} t^{1}\left(t^{2}\right)^{2}+\frac{8}{2}\left(t^{1}\right)^{2} t^{2}+\frac{44}{24} t^{1}+\frac{48}{24} t^{2}+120 \frac{i \zeta(3)}{(2 \pi)^{3}} \\
& +180 q_{1}+\frac{405}{2} q_{1}^{2}+\frac{380}{3} q_{1}^{3}+48 q_{2}+876 q_{1} q_{2}+9772 q_{1}^{2} q_{2} \\
& +46 q_{2}^{2}+3536 q_{1} q_{2}^{2}+\frac{16}{9} q_{2}^{3}+\mathrm{O}\left(q^{4}\right),
\end{aligned}
$$

we can read off the number of lines of degrees $(1,0)$ and $(0,1)$ as 180 and 48 , respectively. Other curves of low degree come with multiplicity $n_{2,0}^{\mathrm{r}}=180, n_{0,2}^{\mathrm{r}}=40$, $n_{1,1}^{\mathrm{r}}=876, n_{1,2}^{\mathrm{r}}=3536$ and $n_{2,1}^{\mathrm{r}}=9672$.

4. As the last two moduli examples we choose a model with five bilinear constraints,

$$
\left(\begin{array}{l||lllll}
\mathbb{P}^{4} & 1 & 1 & 1 & 1 & 1 \\
\mathbb{P}^{4} & 1 & 1 & 1 & 1 & 1
\end{array}\right)_{-32}^{12}
$$

Starting from

$$
\mathscr{L}_{1}=\theta_{1}^{5}-\left(\theta_{1}+\theta_{2}\right)^{5} z_{1}, \quad \mathscr{L}_{2}=\theta_{2}^{5}-\left(\theta_{1}+\theta_{2}\right)^{5} z_{2},
$$


we factorize in five steps, namely

(1) $\mathscr{L}_{1}+\mathscr{L}_{2}=\left(\theta_{1}+\theta_{2}\right) \mathscr{L}_{3}$,

(2) $\theta_{2} \mathscr{L}_{3}-5 \mathscr{L}_{2}=\left(\theta_{1}+\theta_{2}\right) \mathscr{L}_{4}$,

(3) $2 \mathscr{L}_{3}+\mathscr{L}_{4}=\left(\theta_{1}+\theta_{2}\right) \mathscr{L}_{5}$,

(4) $2 \mathscr{L}_{3}-\theta_{1} \mathscr{L}_{5}=\left(\theta_{1}+\theta_{2}\right) \mathscr{L}_{6}$,

(5) $\mathscr{L}_{5}+2 \mathscr{L}_{6}=\left(\theta_{1}+\theta_{2}\right) \mathscr{L}_{7}$, and choose e.g. $\mathscr{L}_{5}$ and $\mathscr{L}_{7}$ as the third and second order differential operators $L_{1}$ and $L_{2}$, respectively. The prepotential as derived from (4.19) reads

$$
\begin{aligned}
F= & \frac{5}{6} \sum_{i}\left(t^{i}\right)^{3}+\frac{10}{2} \sum_{i, j} t^{i}\left(t^{j}\right)^{2}+\frac{20}{24} \sum_{i} t^{i}+32 \frac{i \zeta(3)}{(2 \pi)^{3}}+\sum_{i}\left(50 q_{i}+\frac{25}{4} q_{i}^{2}+\frac{25}{32} q_{i}^{4}\right) \\
& +\sum_{i, j}\left(\frac{650}{2} q_{i} q_{j}+1475 q_{i}^{2} q_{j}+650 q_{i}^{3} q_{j}+\frac{117725}{8} q_{i}^{2} q_{j}^{2}\right)+\mathrm{O}\left(q^{5}\right),
\end{aligned}
$$

here the summation indicated with' ${ }^{\prime}$ is over distinct indices only.

Hence we have in total 100 lines, and the non-zero invariants of curves up to degree four are $n_{1,1}^{\mathrm{r}}=n_{1,3}^{\mathrm{r}}=650, n_{1,2}^{\mathrm{r}}=1475$ and $n_{2,2}^{\mathrm{r}}=29350$.

5. As a three-moduli example we consider

$$
\left(\begin{array}{l||ll}
\mathbb{P}^{3} & 3 & 1 \\
\mathbb{P}^{1} & 0 & 2 \\
\mathbb{P}^{1} & 0 & 2
\end{array}\right)_{-48}^{9}: \quad \begin{gathered}
K^{0}=6 J_{1}^{2} J_{2}+6 J_{1}^{2} J_{3}+3 J_{1} J_{2} J_{3}, \\
c_{2} J_{1}=36, c_{2} J_{2}=c_{2} J_{3}=24
\end{gathered}
$$

for which we have from (3.9)

$$
\begin{aligned}
& \mathscr{L}_{1}=\theta_{1}^{3}-3\left(3 \theta_{1}-2\right)\left(3 \theta_{1}-1\right)\left(\theta_{1}+2 \theta_{2}+2 \theta_{3}\right) z_{1}, \\
& \mathscr{L}_{2}=\theta_{2}^{2}-\left(\theta_{1}+2 \theta_{2}+2 \theta_{3}-1\right)\left(\theta_{1}+2 \theta_{2}+2 \theta_{3}\right) z_{2}, \\
& \mathscr{L}_{3}=\theta_{3}^{2}-\left(\theta_{1}+2 \theta_{2}+2 \theta_{3}-1\right)\left(\theta_{1}+2 \theta_{2}+2 \theta_{3}\right) z_{3} .
\end{aligned}
$$

An independent second order differential operator $L_{4}\left(L_{i}=\mathscr{L}_{i}, i=1,2,3\right)$ can be factorized from the system in the following way:

$$
\mathscr{L}_{1}+\left(16 \theta_{3}-4 \theta_{1}\right) \mathscr{L}_{2}+\left(16 \theta_{2}-4 \theta_{1}\right) \mathscr{L}_{3}=\left(\theta_{1}+2 \theta_{2}+2 \theta_{3}\right) L_{4} .
$$

Due to their symmetry, we have $n_{d_{1}, d_{2}, d_{3}}^{\mathrm{r}}=n_{d_{1}, d_{3}, d_{2}}^{\mathrm{r}}$, and we will thus list the non-zero invariants in Table 3 only for $d_{2} \leqslant d_{3}$ in the range $d_{1}+d_{2}+d_{3} \leqslant 6$. In Section 7 we discuss also the invariants associated to the elliptic curves of this three-moduli example.

The closed formulas (4.18) and (4.19) can be easily evaluated for general toric varities with higher dimensional moduli space provided the generators of the Mori cone $l^{(i)}$ (cf. [16]) and the intersection numbers in the corresponding basis are known. By (4.22) they become a very useful tool for enumerative geometry.

We will demonstrate this in the following for a complete intersection with a six-dimensional Kähler moduli space. 
6. For the six-moduli complete intersection

$$
\left(\begin{array}{l||lll}
\mathbb{P}^{1} & \mathbb{P}^{1} \\
\mathbb{P}^{1} \\
\mathbb{P}^{1} \\
\mathbb{P}^{1} \\
\mathbb{P}^{1}
\end{array} \mid \begin{array}{lll}
0 & 1 & 1 \\
0 & 1 & 1 \\
1 & 1 & 0 \\
1 & 1 & 0 \\
1 & 0 & 1 \\
1 & 0 & 1
\end{array}\right)_{-48}^{6},
$$

a straightforward evaluation of (4.19) immediatly gives the prepotential, which reads up to order four in the $q_{i}$

$$
\begin{aligned}
F= & \frac{1}{3} \sum_{i, j, k} t^{i} t^{j} t^{k}+\sum_{i} t^{i}+48 \frac{i \zeta(3)}{(2 \pi)^{3}}+\sum_{i}\left(16 q_{i}+2 q_{i}^{2}+\frac{16}{27} q_{i}^{3}+\frac{1}{4} q_{i}^{4}\right) \\
& +\sum_{i, j} \frac{1}{2}\left(8 q_{i} q_{j}+q_{i}^{2} q_{j}^{2}\right)+\frac{8}{3} \sum_{i, j, k} q_{i} q_{j} q_{k}+\frac{10}{3} \sum_{i, j, k, l} q_{i} q_{j} q_{k} q_{l} \\
& +\mathrm{O}\left(q^{5}\right)
\end{aligned}
$$

here again the primed sums are to be taken over distinct indices only. From this expression we can readily predict the number of lines $n_{1,0,0,0,0,0}^{\mathrm{r}}=8$, curves of multi-degree $n_{1,1,0,0,0,0}^{\mathrm{r}}=8, n_{1,1,1,0,0,0}^{\mathrm{r}}=16$ and $n_{1,1,1,1,0,0}^{\mathrm{r}}=80$. Other invariants follow by permutation. There are no curves of degree $(2,0,0,0,0,0),(3,0,0,0,0,0)$ and $(4,0,0,0,0,0)$. The expressions for the Yukwa couplings and the Weil-Peterson metric in the large radius expansions follow from (4.11) and (4.12).

We use our next examples to demonstrate that the analysis of the large complex respectively the Kähler structure limit in the previous section and formulas (4.18), (4.19) have an application also to embeddings in toric varieties with Gorenstein singularities.

7. We consider first the hypersurface of degree 18 in the weighted projective space $\mathbb{P}^{4}[6,6,3,2,1]$, which reads in the shorthand notation (2.1) as $\left(\mathbb{P}^{4}[6,6,3,2,1] \| 18\right)_{-144}^{7}$. This model was discussed qualitatively in the context of topology changes by flops in [31], but it was not solved.

Generally, for quasi-smooth hypersurfaces and complete intersections in weighted $\mathbb{P}^{n}$, the singularities of the ambient space intersect the Calabi-Yau hypersurface $X$ in sets of codimension two and three, see e.g. [32,25].

In our example we have a singular curve $C_{1}$ in $X$ with a $\mathbb{Z}_{2}$ action on its normal bundle, which lies inside the singular stratum where the third and the fifth homogeneous coordinate of the weighted projective space are set to zero. The resolution introduces a $\mathbb{P}^{1}$ fibration over that curve, which gives rise to one exceptional divisor $D_{1}$. Similarly, the singular stratum of the weighted projective space where $z_{4}=z_{5}=0$ intersects $X$ in a curve $C_{2}$ with a $\mathbb{Z}_{3}$ action on the normal bundle, whose resolution leads to a fibration with a $\mathbb{P}^{1} \wedge \mathbb{P}^{1}$ sphere-tree over that 
Table 3

\begin{tabular}{lclr}
\hline$\left(d_{1}, d_{2}, d_{3}\right)$ & $n_{d_{1}, d_{2}, d_{3}}^{\mathrm{r}}$ & $\left(d_{1}, d_{2}, d_{3}\right)$ & $n_{d_{1}, d_{2}, d_{3}}^{\mathrm{r}}$ \\
\hline$(0,0,1)$ & 18 & $(1,1,1)$ & 1512 \\
$(0,1,1)$ & 60 & $(2,2,2)$ & 621000 \\
$(0,2,2)$ & 48 & $(1,1,2)$ & 1512 \\
$(0,3,3)$ & 60 & $(1,1,3)$ & 216 \\
$(0,1,2)$ & 18 & $(1,2,2)$ & 7128 \\
$(0,2,3)$ & 18 & $(1,2,3)$ & 7128 \\
$(0,3,4)$ & 18 & $(2,0,1)$ & 2106 \\
$(1,0,0)$ & 216 & $(4,0,2)$ & 414720 \\
$(2,0,0)$ & 216 & $(2,1,1)$ & 28232 \\
$(3,0,0)$ & 48 & $(2,1,2)$ & 85212 \\
$(4,0,0)$ & 216 & $(2,1,3)$ & 28232 \\
$(5,0,0)$ & 216 & $(3,0,1)$ & 17856 \\
$(6,0,0)$ & 48 & $(3,0,2)$ & 17856 \\
$(1,0,1)$ & 216 & $(3,1,1)$ & 656952 \\
$(2,0,2)$ & 48 & $(3,1,2)$ & 2984904 \\
$(3,0,3)$ & & $(4,0,1)$ & 95094 \\
& & $(4,1,1)$ & 8757828 \\
& & $(5,0,1)$ & 41472 \\
\hline
\end{tabular}

curve, hence two irreducible divisors $D_{2}, D_{3}$ on the resolved space. The singular curves meet in three $\mathbb{Z}_{6}$ singular points inside the stratum $z_{3}=z_{4}=z_{5}=0$, whose resolutions support one exceptional divisor for each point, $\tilde{D}_{4}, \tilde{D}_{5}, \tilde{D}_{6}$. Hence we have, including the divisor $D_{0}$ from the Picard group of the singular space, a seven-moduli case.

The toric description of the mirror pair in terms of reflexive polyhedra was given in [26], and reviewed in [16,31]. Associated to the manifold $X$ and its mirror $\mathrm{X}^{*}$ are the simplicial polyhedra $\Delta$ and $\Delta^{*}$, which are defined as the convex hull of the following points:

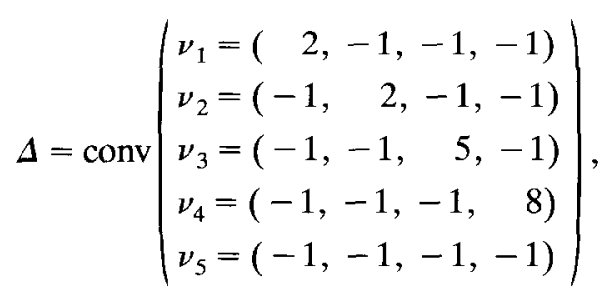




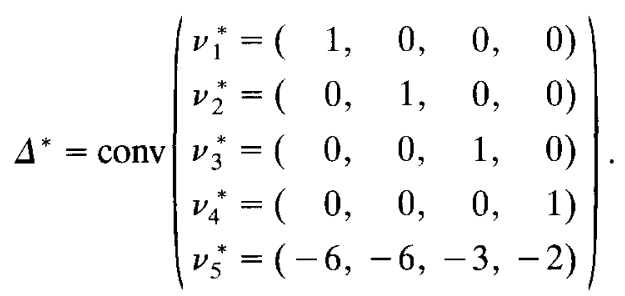

Five points in the dual polyhedron $\Delta^{*}$, namely the point in its interior: $\nu_{0}^{*}=$ $(0,0,0,0)$, and the points on edges of codimension three: $\nu_{6}^{*}=(-3,-3,-1,-1)$, $\nu_{7}^{*}=(-2,-2,-1,0), \nu_{8}^{*}=(-4,-4,-2,-1)$, and on the face of codimension two: $\nu_{9}^{*}=(-1,-1,0,0)$, can be identified with the (exceptional) divisors of the Calabi-Yau hypersurface X. Only for these divisors the toric description of the Kähler cone and its dual, the Mori cone, can be applied ${ }^{13}$. We prefer to work with the Mori cone, as its generators are directly related to the expressions for the PF equations and the definition of the local coordinates for the large complex structure limit.

The $\mathbb{Z}_{6}$ singularity of type $\mathbb{C}^{3} / G$ with $G=\operatorname{diag}\left(\alpha, \alpha^{2}, \alpha^{3}\right)\left(\alpha=\mathrm{e}^{2 \pi i / 6}\right)$ is described in this construction by the three-dimensional cone $\Sigma$, spanned from the origin $\nu_{0}^{*}$ by $\left\{\nu_{3}^{*}, \nu_{4}^{*}, \nu_{5}^{*}\right\}$. The cone $\Sigma$ is not basic, i.e. the three vectors $\left\{\nu_{3}^{*}, \nu_{4}^{*}, \nu_{5}^{*}\right\}$ do not generate the lattice $\Sigma \cap \mathrm{M}$, with $\mathrm{M}=\mathrm{N}=\left\{\left(n_{1}, n_{2}, n_{3}, n_{4}\right) \mid n_{i}\right.$ $\in \mathbb{Z}$. The volume of $\sigma \cap \Delta^{*}=\operatorname{conv}\left(\nu_{0}^{*}, \nu_{3}^{*}, \nu_{4}^{*}, \nu_{5}^{*}\right)$ is six, which is the order of the defining group for this singularity. $\Sigma \cap \Delta^{*}$ contains the point $\nu_{6}^{*}$ on the edge $\left\{\nu_{3}^{*}, \nu_{5}^{*}\right\}$, which can be identified with the exceptional divisor $D_{1}$ in the resolution over the curve $C_{1}$, as well as the points $\nu_{7}^{*}, \nu_{8}^{*}$ on the edge $\left\{\nu_{4}^{*}, \nu_{5}^{*}\right\}$, which correspond to $D_{2}, D_{3}$. On the other hand, the point $\nu_{9}^{*}$ inside the triangle with corners $\left\{\nu_{3}^{*}, \nu_{4}^{*}, \nu_{5}^{*}\right\}$ corresponds to only one divisor $D_{4}$ in the resolution of three $\mathbb{Z}_{6}$ singular points. In the formula for the dimension of $H^{1,1}(X)[26]$, the multiplicity three is taken into account by an additional term, which multiplies the interior points in the three-dimensional cone $\Sigma$ with the number of points on the interior of its two-dimensional dual, which in this case is spanned by the origin in $\Delta$ and $\left\{\nu_{1}, \nu_{2}\right\}$. So it contains two points $\delta_{1}=(1,0,-1,-1)$ and $\delta_{2}=(0,1,1,-1)$.

There are five different canonical resolutions of the $\mathbb{C}^{3} / \mathbb{Z}_{6}$ singularity, corresponding to the five possible subdivisons of the cone $\Sigma$ into six basic cones of

\footnotetext{
${ }^{13}$ This technical complication could have been avoided if we had instead considered the model $\left(\mathbb{P}^{4}[1,2,3,12,18] \| 36\right)_{-360}^{5}$, which has same type of singularities, but all divisors can be described by a toric geometry in this case.
} 
volume one, which are spanned from $\nu_{0}^{*}$ by
$\mathrm{A}:\left\{\nu_{3}^{*}, \nu_{6}^{*}, \nu_{9}^{*}\right\}, \quad\left\{\nu_{6}^{*}, \nu_{5}^{*}, \nu_{8}^{*}\right\}, \quad\left\{\nu_{8}^{*}, \nu_{9}^{*}, \nu_{6}^{*}\right\}$,
$\left\{\nu_{8}^{*}, \nu_{7}^{*}, \nu_{9}^{*}\right\}, \quad\left\{\nu_{7}^{*}, \nu_{4}^{*}, \nu_{9}^{*}\right\}, \quad\left\{\nu_{4}^{*}, \nu_{3}^{*}, \nu_{9}^{*}\right\}$;
$\mathrm{B}:\left\{\nu_{3}^{*}, \nu_{6}^{*}, \nu_{8}^{*}\right\}, \quad\left\{\nu_{6}^{*}, \nu_{5}^{*}, \nu_{8}^{*}\right\}, \quad\left\{\nu_{8}^{*}, \nu_{9}^{*}, \nu_{3}^{*}\right\}$,
$\left\{\nu_{8}^{*}, \nu_{7}^{*}, \nu_{9}^{*}\right\}, \quad\left\{\nu_{7}^{*}, \nu_{4}^{*}, \nu_{9}^{*}\right\}, \quad\left\{\nu_{4}^{*}, \nu_{3}^{*}, \nu_{9}^{*}\right\}$;
$\mathrm{C}:\left\{\nu_{3}^{*}, \nu_{6}^{*}, \nu_{9}^{*}\right\}, \quad\left\{\nu_{6}^{*}, \nu_{5}^{*}, \nu_{9}^{*}\right\}, \quad\left\{\nu_{5}^{*}, \nu_{8}^{*}, \nu_{9}^{*}\right\}$,
$\left\{\nu_{8}^{*}, \nu_{7}^{*}, \nu_{9}^{*}\right\}, \quad\left\{\nu_{7}^{*}, \nu_{4}^{*}, \nu_{9}^{*}\right\}, \quad\left\{\nu_{4}^{*}, \nu_{3}^{*}, \nu_{9}^{*}\right\}$;
$\mathrm{D}:\left\{\nu_{3}^{*}, \nu_{6}^{*}, \nu_{9}^{*}\right\}, \quad\left\{\nu_{6}^{*}, \nu_{5}^{*}, \nu_{8}^{*}\right\}, \quad\left\{\nu_{8}^{*}, \nu_{7}^{*}, \nu_{6}^{*}\right\}$,
$\left\{\nu_{7}^{*}, \nu_{9}^{*}, \nu_{6}^{*}\right\}, \quad\left\{\nu_{7}^{*}, \nu_{4}^{*}, \nu_{9}^{*}\right\}, \quad\left\{\nu_{4}^{*}, \nu_{3}^{*}, \nu_{9}^{*}\right\}$;
$\mathrm{E}:\left\{\nu_{3}^{*}, \nu_{6}^{*}, \nu_{9}^{*}\right\}, \quad\left\{\nu_{6}^{*}, \nu_{5}^{*}, \nu_{8}^{*}\right\}, \quad\left\{\nu_{8}^{*}, \nu_{7}^{*}, \nu_{6}^{*}\right\}$,
$\left\{\nu_{7}^{*}, \nu_{4}^{*}, \nu_{6}^{*}\right\}, \quad\left\{\nu_{4}^{*}, \nu_{9}^{*}, \nu_{6}^{*}\right\}, \quad\left\{\nu_{4}^{*}, \nu_{3}^{*}, \nu_{9}^{*}\right\}$

for the different resolutions. The generators of the Mori cone $l^{(i)}$ and the Picard-Fuchs system in the corresponding large complex structure coordinates $z_{i}$ can be obtained for each subdivision by the methods described in [16]. They will of course depend on the subdivision.

The subdivisions are connected by flops, e.g. subdivision B is obtained from subdivision $\mathrm{A}$ by the flop, which blows down the $\mathbb{P}^{1}$ represented by the subcone spanned from $\nu_{0}^{*}$ by $\left\{\nu_{6}^{*}, \nu_{9}^{*}\right\}$ in subdivision $\mathrm{A}$ and subsequently blows up the $\mathbb{P}^{1}$ associated with the new subcone spanned from $\nu_{0}^{*}$ by $\left\{\nu_{3}^{*}, \nu_{8}^{*}\right\}$ in subdivision $\mathrm{B}$. We therefore expect simple relations of the generators of the Mori cones and large complex structure coordinates for all subdivisions to those of subdivision A. After extending the vectors $\nu_{i}{ }^{*}$ to $\vec{\nu}_{i}{ }^{*}=\left(1, \nu_{i}{ }^{*}\right)$, the generators of the Mori cone can be constructed as described in $[30,16]$ to yield

$$
\begin{aligned}
& l_{\AA}^{(1)}=(-3 ; 1,1,0,0,-1, \quad 1,0,1,0), \\
& l_{\AA}^{(2)}=(0 ; 0,0,1,0, \quad 0,-1,0,1,-1), \\
& l_{\AA}^{(3)}=(0 ; 0,0,0,0,1,-1,0,-1,1), \\
& l_{\AA}^{(4)}=(0 ; 0,0,0,0,0,1,1,-1,-1), \\
& l_{\AA}^{(5)}=(0 ; 0,0,0,1, \quad 0,0,-2,1,0) .
\end{aligned}
$$

They define the large complex structure variables as $z_{1}^{(\mathrm{A})}=-a^{l_{\mathrm{A}}^{(1)}}$ and $z_{i}^{(\mathrm{A})}=a^{l_{\mathrm{A}}^{(\mathrm{i})}}$ for $i=2,3,4,5$. As a next step, we set up the $\bar{\Delta}^{*}$-hypergeometric system (see e.g. [26,16] for details). Every linear relation among the $\bar{\nu}_{i}{ }^{*}$, not just the ones encoded in the $l_{\mathrm{A}}^{(i)}$ (cf. (3.2)), defines a linear differential operator of this system, which is satisfied by all periods. It should be mentioned again that this system is not equivalent to the Picard-Fuchs system; the nontrivial task will be to find, possibly after factorization, a minimal systems of differential operators which uniquely determine the $(2 k+2)$-dimensional period vector in the domain containing the 
points $z^{(\mathrm{A}, \ldots, \mathrm{E})}=0$. These systems are equivalent to the first order Gauss-Manin systems in these domains, and all other differential operators are elements of the local left ideal associated to it (cf. Section 4). As we will see, the minimal number of differential operators generating this ideal can vary from domain to domain. The consideration of the rings $\mathrm{R}^{(\mathrm{A}, \ldots, \mathrm{E})}$ will be essential in order to find these generators.

From the fact that the free polynomial ring $\mathbb{C}\left[\theta_{z}^{(A)}\right]$ has to be truncated at degree two from fifteen to five elements by the principal part of the linear differential operators, we know that there must be ten second order differential operators. Indeed we find that the linear relations between the sites of the points in $\bar{\Delta}^{*}$ which are expressed by the following vectors: $l_{\mathrm{A}}^{(2)}, l_{\mathrm{A}}^{(3)}, l_{\mathrm{A}}^{(4)}, l_{\mathrm{A}}^{(5)}, l_{\mathrm{A}}^{(2)}+l_{\mathrm{A}}^{(3)}, l_{\mathrm{A}}^{(2)}+l_{\mathrm{A}}^{(4)}$, $l_{\mathrm{A}}^{(3)}+l_{\mathrm{A}}^{(4)}, l_{\mathrm{A}}^{(4)}+l_{\mathrm{A}}^{(5)}, l_{\mathrm{A}}^{(3)}+l_{\mathrm{A}}^{(4)}+l_{\mathrm{A}}^{(5)}$, define directly nine second order differential operators of the $\Delta^{*}$-hypergeometric system (cf. [26,16],

$$
\begin{array}{ll}
L_{1}^{(\mathrm{A})}=\theta_{a_{3}} \theta_{a_{8}}-a^{l\left(l_{\AA}^{(2)}\right.} \theta_{a_{6}} \theta_{a_{9}}, & L_{2}^{(\mathrm{A})}=\theta_{a_{5}} \theta_{a_{9}}-a^{l_{\AA}^{(3)}} \theta_{a_{6}} \theta_{a_{8}}, \\
L_{3}^{(\mathrm{A})}=\theta_{a_{6}} \theta_{a_{7}}-a^{l_{\AA}^{(4)}} \theta_{a_{8}} \theta_{a_{9}}, & L_{4}^{(\mathrm{A})}=\theta_{a_{4}} \theta_{a_{8}}-a^{l_{\AA}^{(5)}}\left(\theta_{a_{7}}-1\right) \theta_{a_{7}}, \\
L_{5}^{(\mathrm{A})}=\theta_{a_{3}} \theta_{a_{5}}-a^{l\left(l_{\AA}^{(2)}+l_{\AA}^{(3)}\right.}\left(\theta_{a_{6}}-1\right) \theta_{a_{6}}, & L_{6}^{(\mathrm{A})}=\theta_{a_{3}} \theta_{a_{7}}-a^{l_{\AA}^{(2)}+l_{\AA}^{(4)}}\left(\theta_{a_{9}}-1\right) \theta_{a_{9}}, \\
L_{7}^{(\mathrm{A})}=\theta_{a_{5}} \theta_{a_{7}}-a^{l(3)+l_{\AA}^{(4)}}\left(\theta_{a_{8}}-1\right) \theta_{a_{8}}, & L_{8}^{(\mathrm{A})}=\theta_{a_{4}} \theta_{a_{6}}-a^{l_{\AA}^{(4)}+l_{\AA}^{(5)}} \theta_{a_{7}} \theta_{a_{9}}, \\
L_{9}^{(\mathrm{A})}=\theta_{a_{4}} \theta_{a_{5}}-a^{l_{\AA}^{(3)}+l_{\AA}^{(4)}+l_{\AA}^{(5)} \theta_{a_{7}} \theta_{a_{8}} .} &
\end{array}
$$

The logarithmic derivatives $\theta_{a_{i}}:=a_{i} \partial / \partial_{a_{i}}$ can be readily translated to logarithmic derivatives in the $z_{i}$ by the identity $\theta_{a_{i}}=\sum_{k=1}^{h} l_{i}^{(k)} \theta_{k}$. Via this relation we also see that we can factor a $\theta_{1}$ operator from the differential operator for $l_{\mathrm{A}}^{(1)}+l_{\mathrm{A}}^{(3)}$ to yield the tenth second order differential operator $L_{10}^{(\mathrm{A})}$,

$$
\begin{aligned}
\theta_{1} L_{10}^{(\mathrm{A})} & =\theta_{a_{1}} \theta_{a_{2}} \theta_{a_{9}}-a^{l_{\AA}^{(1)}+l_{\AA}^{(3)}}\left(\theta_{a_{0}}-3\right)\left(\theta_{a_{0}}-2\right)\left(\theta_{a_{0}}-1\right) \\
& =\theta_{1}\left[\theta_{1}\left(\theta_{2}-\theta_{3}+\theta_{4}\right)+3 z_{1} z_{2}\left(3 \theta_{1}+2\right)\left(3 \theta_{1}+1\right)\right]
\end{aligned}
$$

While $\mathbb{C}\left[\theta_{z}\right]$ is truncated by the ideal $I_{s}\left(\theta_{z}\right), s=1, \ldots, 10$, obtained from (5.25), (5.26), to five elements at degree two, one can easily check that we still have two elements at degree three. So we need one further independent 3rd order differential operator. We find e.g. that $l_{\mathrm{A}}^{(1)}$ leads, after factorization of $\theta_{1}$, to the operator

$$
\begin{aligned}
L_{11}^{(\mathrm{A})}= & \theta_{1}\left(\theta_{1}-\theta_{2}-\theta_{3}+\theta_{4}\right)\left(\theta_{1}+\theta_{2}-\theta_{3}-\theta_{4}+\theta_{5}\right) \\
& +3 z_{1}\left(3 \theta_{1}+2\right)\left(3 \theta_{1}+1\right)\left(\theta_{1}-\theta_{3}\right)
\end{aligned}
$$

which truncates $\mathbb{C}\left[\theta_{z}\right]$ into one element at degree three and no element at any higher order. Now we can construct, as in Section 4 , via the ring $R^{(A)}$ the period vector and calculate the prepotential explicitly. The classical intersection part and 
the corresponding expression for the deformed prepotential can be found most simply from (4.18) to be, up to terms of order $q^{4}$,

$$
\begin{aligned}
F^{(\mathrm{A})}= & \frac{18}{3 !}\left(t^{1}\right)^{3}+\frac{9}{2}\left(t^{1}\right)^{2} t^{2}+\frac{3}{2} t^{1}\left(t^{2}\right)^{2}+\frac{21}{2}\left(t^{1}\right)^{2} t^{3}+9 t^{1} t^{2} t^{3} \\
& +\frac{3}{2}\left(t^{2}\right)^{2} t^{3}+\frac{21}{2} t^{1}\left(t^{3}\right)^{2}+\frac{9}{2} t^{2}\left(t^{3}\right)^{2}+\frac{21}{3 !}\left(t^{3}\right)^{3}+\frac{12}{2}\left(t^{1}\right)^{2} t^{4} \\
& +6 t^{1} t^{2} t^{4}+12 t^{1} t^{3} t^{4}+6 t^{2} t^{3} t^{4}+\frac{12}{2}\left(t^{3}\right)^{2} t^{4}+\frac{6}{2} t^{1}\left(t^{4}\right)^{2} \\
& +\frac{6}{2} t^{3}\left(t^{4}\right)^{2}+\frac{6}{2}\left(t^{1}\right)^{2} t_{5}+3 t^{1} t^{2} t^{5}+6 t^{1} t^{3} t^{5}+3 t^{2} t^{3} t^{5}+\frac{6}{2}\left(t^{3}\right)^{2} t^{5} \\
& +3 t^{1} t^{4} t^{5}+3 t^{3} t^{4} t^{5}+\frac{1}{24}\left(72 t^{1}+36 t^{2}+78 t^{3}+48 t^{4}+24 t^{5}\right) \\
& +144 \frac{i \zeta(3)}{(2 \pi)^{3}}+\left(27 q_{1}+3 q_{2}+3 q_{3}+3 q_{4}\right) \\
& +\left(\frac{3 q_{2}^{2}}{8}-\frac{405 q_{1}^{2}}{8}+108 q_{1} q_{3}+\frac{3 q_{3}^{2}}{8}-6 q_{2} q_{4}+\frac{3 q_{4}^{2}}{8}+3 q_{4} q_{5}\right) \\
& +\left(244 q_{1}^{3}+\frac{q_{2}^{3}}{9}+81 q_{1}^{2} q_{3}+27 q_{1} q_{2} q_{3}\right. \\
& \left.+\frac{q_{3}^{3}}{9}+27 q_{1} q_{3} q_{4}+3 q_{2} q_{3} q_{4}+\frac{q_{4}^{3}}{9}-6 q_{2} q_{4} q_{5}\right)
\end{aligned}
$$

We can next use the mirror hypothesis to interpret the coefficients of the cubic terms in $t^{i}$ of (5.28) as triple intersection numbers of $\mathrm{H}_{2}(\mathrm{X}, \mathbb{Z})$. Note that all triple intersection numbers are positive, as expected in the bases $J_{i}$ which generates the Kähler cone. The linear and the quadratic terms in the $t^{i}$ obtained by (4.20), (4.21) can be compared with the classical calculation of these topological numbers performed e.g. in [25]. These formulas relate all topological data and the $l^{(i)}$ and provide an excellent check for our calculation. As it stands, the classical part of $F^{(\mathrm{A})}$ does not refer to the basis of divisors $D_{i}$ for which these intersection numbers can be directly obtained by the formulas for resolved complete intersections in toric varieties summarized e.g. in $[16,25]$. To make the comparison we can easily transform to that basis by passing to the variables $t_{\mathrm{D}_{\mathrm{i}}}{ }^{14}$ via $t^{i}=\delta^{i, 1} t_{\mathrm{D}_{0}}+$ $\sum_{j=1}^{k-1} l_{\mathrm{A}, j+5}^{(i)} t_{\mathrm{D}_{j}}$, i.e. from the $z_{i}^{(\mathrm{A})}$ variables to the complex structure deformation

\footnotetext{
${ }^{14}$ Note that this reparametrization is quite different from the transformations into different domains, discussed below, because we still keep $z_{i}=0$ as the point around which we expand our solutions of the $\mathrm{PF}$ equation.
} 
variables which occur explicitly in the Laurent polynomial, namely $1 / a_{0}^{3}, a_{6}, a_{7}$, $a_{8}, a_{9}$. This leads to the prediction

$$
\begin{aligned}
F_{\mathrm{cl}}^{(\mathrm{A})}= & \frac{18}{3 !} t_{D_{0}}^{3}-\frac{6}{2} t_{D_{0}} t_{D_{1}}^{2}-\frac{1}{2} t_{D_{1}}^{3}-\frac{6}{2} t_{D_{0}} t_{D_{2}}^{2}-\frac{3}{2} t_{D_{1}}^{2} t_{D_{3}}+3 t_{D_{0}} t_{D_{2}} t_{D_{3}}-3 t_{D_{0}} t_{D_{3}}^{2} \\
& -\frac{3}{2} t_{D_{1}} t_{D_{3}}^{2}-\frac{3}{3 !} t_{D_{3}}^{3}-\frac{3}{2} t_{D_{1}}^{2} t_{D_{4}}-\frac{6}{2} t_{D_{2}}^{2} t_{D_{4}}+3 t_{D_{1}} t_{D_{3}} t_{D_{4}}+3 t_{D_{2}} t_{D_{3}} t_{D_{4}} \\
& -\frac{3}{2} t_{D_{3}}^{2} t_{D_{4}}-\frac{3}{2} t_{D_{1}} t_{D_{4}}^{2}-\frac{3}{2} t_{D_{3}} t_{D_{4}}^{2}+\frac{21}{3 !} t_{D_{4}}^{3}+\frac{1}{24}\left(72 t_{D_{0}}+6 t_{D_{1}}+6 t_{D_{3}}-6 t_{D_{4}}\right) \\
& +144 \frac{i \zeta(3)}{(2 \pi)^{3}},
\end{aligned}
$$

which is in agreement with the formulas of the classical intersections in the basis of the $D_{i}$. Note, however, that all intersection numbers among the divisors on the triangle with corners $\left\{\nu_{3}^{*}, \nu_{4}^{*}, \nu_{5}^{*}\right\}$ are multiplied by three in $F_{\mathrm{cl}}^{(\mathrm{A})}$. This is due to the fact that the $\mathbb{Z}_{6}$ fixed point has multiplicity three and the toric description of this singularity by $\Sigma$ refers to the symmetric combination $D_{4}=\tilde{D}_{4}+\tilde{D}_{5}+\tilde{D}_{6}$ of the exeptional divisors over the three singular points on $\mathrm{X}$, with

$$
K_{D_{i} \tilde{D}_{j} \tilde{D}_{k}}^{Q}= \begin{cases}7 & \text { if } i=j=k \\ 0 & \text { otherwise. }\end{cases}
$$

To investigate the theory in different domains of the moduli space we note the simple transformation property of the $\theta_{i}$ operators in the Picard-Fuchs system, i.e. for $z_{i}^{(\mathrm{B})}=\prod_{j}\left(z_{j}^{(\mathrm{A})}\right)^{m_{i, j} \leftrightarrow l_{\mathrm{B}}^{(i)}}=\sum_{j} m_{i, j} l_{\mathrm{A}}^{(i)}$, the $\theta_{i}$ transform as $\theta_{i}^{(\mathrm{A})}=\sum_{j} m_{j, i} \theta_{j}^{(\mathrm{B})}$. We are interested in transformations which lead outside the Mori cone, i.e. in which not all entries of the matrix $m$ are positive. A quick look at (5.25) and (5.26) reveals that the possibilities for such transformations are rather restricted if we insist on a completely degenerate large complex structure limit in the new domain $\mathrm{B}$, i.e. a ring structure $\mathrm{R}^{\mathrm{B})}$ with the properties discussed in Section 4. For instance, we cannot just invert $z_{5}$ (which would correspond to the replacement $l_{\mathrm{A}}^{(5)} \rightarrow-l_{\mathrm{A}}^{(5)}$ ) without generating inhomogeneous (in $\theta$ ) terms in $I\left(\theta_{z}\right)$, which is incompatible with the required ring structure of $\mathrm{R}$. It is easy to see that the only possibilities are to invert $z_{2}, z_{3}, z_{4}$ and $z_{4} z_{5}$, accompanied by transformations of the other variables. These transformations correspond to the flops leading to the coordinate patches described by the Mori cone of subdivisions B, C, D and E. They form part of the secondary fan as described in [30].

We start with the inversion of $z_{2}$, which leads to subdivision $\mathrm{B}$ for which the generators of the Mori cone read $l_{\mathrm{B}}^{(1)}=l_{\mathrm{A}}^{(1)}, l_{\mathrm{B}}^{(2)}=-l_{\mathrm{A}}^{(2)}, l_{\mathrm{B}}^{(3)}=l_{\mathrm{A}}^{(2)}+l_{\mathrm{A}}^{(3)}, l_{\mathrm{B}}^{(4)}=l_{\mathrm{A}}^{(2)}+$ $l_{\mathrm{A}}^{(4)}$ and $l_{\mathrm{B}}^{(5)}=l_{\mathrm{A}}^{(5)}$. Note that the matrix $m$ for a single flop squares to unity, and the principal parts of the transformed system truncates $\mathbb{C}\left[\theta_{z}\right]$ at degree two to five elements, but two elements remain at degree three. We can remedy the situation by adding the operator associated to $l_{\mathrm{A}}^{(1)}+l_{\mathrm{A}}^{(2)}+l_{\mathrm{A}}^{(3)}$ to our system (5.25)-(5.27),

$$
\theta_{1} L_{12}^{(\mathrm{A})}=\theta_{a_{1}} \theta_{a_{2}} \theta_{a_{3}} \theta_{a_{8}}-a^{l_{\mathrm{A}}^{(1)}+l_{\mathrm{A}}^{(2)}+l_{\mathrm{A}}^{(3)}}\left(\theta_{a_{0}}-3\right)\left(\theta_{a_{0}}-2\right)\left(\theta_{a_{0}}-1\right) \theta_{a_{6}},
$$


which factorizes to a third order operator $L_{12}^{(\mathrm{A})}$. For subdivision $\mathrm{C}: l_{\mathrm{C}}^{(1)}=l_{\mathrm{A}}^{(1)}+l_{\mathrm{A}}^{(3)}$, $l_{\mathrm{C}}^{(2)}=l_{\mathrm{A}}^{(2)}+l_{\mathrm{A}}^{(3)}, l_{\mathrm{C}}^{(3)}=-l_{\mathrm{A}}^{(3)}, l_{\mathrm{C}}^{(4)}=l_{\mathrm{A}}^{(3)}+l_{\mathrm{A}}^{(4)}$ and $l_{\mathrm{C}}^{(5)}=l_{\mathrm{A}}^{(5)}$, no further operator has to be added. To complete our system w.r.t. subdivision $\mathrm{D}: l_{\mathrm{D}}^{(1)}=l_{\mathrm{A}}^{(1)}, l_{\mathrm{D}}^{(2)}=l_{\mathrm{A}}^{(2)}+l_{\mathrm{A}}^{(4)}$, $l_{\mathrm{D}}^{(3)}=l_{\mathrm{A}}^{(3)}+l_{\mathrm{A}}^{(4)}, l_{\mathrm{D}}^{(4)}=-l_{\mathrm{A}}^{(4)}, l_{\mathrm{D}}^{(5)}=l_{\mathrm{A}}^{(4)}+l_{\mathrm{A}}^{(5)}$, and $\mathrm{E}: l_{\mathrm{E}}^{(1)}=l_{\mathrm{A}}^{(1)}, l_{\mathrm{E}}^{(2)}=2 l_{\mathrm{A}}^{(1)}+l_{\mathrm{A}}^{(2)}+l_{\mathrm{A}}^{(5)}$, $l_{\mathrm{E}}^{(3)}=l_{\mathrm{A}}^{(3)}+l_{\mathrm{A}}^{(4)}, l_{\mathrm{E}}^{(4)}=l_{\mathrm{A}}^{(5)}, l_{\mathrm{E}}^{(5)}=-l_{\mathrm{A}}^{(4)}-l_{\mathrm{A}}^{(5)}$, we have to add three third order operators $L_{13}^{(\mathrm{A})}, L_{14}^{(\mathrm{A})}, L_{15}^{(\mathrm{A})}$ :

$$
\begin{aligned}
& \theta_{1} L_{13}^{(\mathrm{A})}=\theta_{a_{1}} \theta_{a_{2}} \theta_{a_{6}} \theta_{a_{7}}-a^{l_{\AA}^{(1)}+l_{\AA}^{(2)}+l_{\AA}^{(4)}}\left(\theta_{a_{0}}-3\right)\left(\theta_{a_{0}}-2\right)\left(\theta_{a_{0}}-1\right) \theta_{a_{8}}, \\
& \theta_{1} L_{14}^{(\mathrm{A})}=\theta_{a_{1}} \theta_{a_{2}} \theta_{a_{4}} \theta_{a_{6}}-a^{l_{\AA}^{(1)}+l_{\AA}^{(3)}+l_{\AA}^{(4)}+l_{\AA}^{(5)}}\left(\theta_{a_{0}}-3\right)\left(\theta_{a_{0}}-2\right)\left(\theta_{a_{0}}-1\right) \theta_{a_{7}}, \\
& L_{15}^{(\mathrm{A})}=\theta_{a_{3}} \theta_{a_{4}} \theta_{a_{5}}-a^{l_{\AA}^{(2)}+l_{\AA}^{(3)}+2 l_{\AA}^{(4)}+l_{\AA}^{(5)}} \theta_{a_{8}}\left(\theta_{a_{9}}-1\right) \theta_{a_{9}} .
\end{aligned}
$$

The system $L_{1}^{(\mathrm{A})}, \ldots, L_{15}^{(\mathrm{A})}$ contains the information which is necessary to extract the prepotential in all large complex structure regions. The basis of solutions given in (4.10) can now be obtained explicitly in all regions. As they are all solutions to the same system of Picard-Fuchs equations, expressed in different patches of the moduli space, they are analytic continuations of each other with trivial monodromy. Especially the prepotentials $F^{(\mathrm{A}, \mathrm{B}, \mathrm{C}, \mathrm{D}, \mathrm{E})}$ encoded in the $2 k+2$ component of (4.10) is the same analytic function whose expansion in the domains corresponding to the different resolutions can be evaluated with the attached program. Also, for $F^{(\mathrm{B}, \mathrm{C}, \mathrm{D}, \mathrm{E})}$ we obtain, after transformation via $t^{i}=\delta^{i, 1} t_{D_{0}}+\sum_{j=1}^{k=1} l_{j+5}^{(i)} t_{D_{j}}$ to the $D_{i}$ basis, the classical intersection numbers as calculated e.g. by the formulas of [16], with the enhancement factor three for intersections among divisors on the triangle.

We also checked, up to order eight, that the expansions of $F^{\text {(A,B,C,D,E) }}$ are compatible with an integer expansion for $n^{\mathrm{r}}$ in (4.22). E.g. from $F^{(\mathrm{A})}$ we obtain, up to degree eight, instanton contributions $n_{i, 0,0,0,0}^{\mathbf{r}}$ with alternating sign, see Table 4 . We can read from the prepotential that

$$
n_{0,1,0,0,0}^{r}=n_{0,0,1,0,0}^{r}=n_{0,0,0,1,0}^{r}=n_{0,0,0,1,1}^{r}=3 .
$$

There are no curves of degree $(0, i, 0,0,0),(0,0, i, 0,0),(0,0,0, i, 0)$ and $(0,0,0, i, i)$ for $i>1$ and no curve $(0,0,0,0, i)$ for all $i$. By comparing with the expansions in the different regions we find that the three rational curves with degrees $(0,1,0,0,0),(0,0,1,0,0),(0,0,0,1,0)$ and $(0,0,0,1,1)$, respectively, are those which are shrunk to zero volume and whose corresponding invariant changes sign under the process of the four possible flop operations interrelating them, starting from resolution $\mathrm{A}$ (cf. the discussion in [31]).

For certain directions in the Kähler cone, e.g. $(i, 0, i, 0,0)$, one has a periodicity in the invariants $n^{r}=108,108,144, \ldots$ as in Sections $5.1-5.3$ and 5.5 before. It is remarkable that in all cases where we obtain periodicity, always one of the

Table 4

\begin{tabular}{lllllllll}
\hline$i$ & 1 & 2 & 3 & 4 & 5 & 6 & 7 & 8 \\
\hline$n_{i, 0,0,0,0}^{\mathrm{r}}$ & 27 & -54 & 243 & -1728 & 15255 & -153576 & 1696086 & -20053440 \\
\hline
\end{tabular}


numbers, the third in the scheme $a, a, b, \ldots$, is the negative of the Euler number of the manifold $\mathrm{X}$.

8. So far we have considered complete intersections in nonsingular ambient spaces, and in the last example as well as in [16], hypersurfaces in ambient spaces with Gorenstein singularities. Let us investigate in the following the more general situation of complete intersections of codimension $n-3$ in an $n$-dimensional singular ambient space, and take the following hypersurface and complete intersection two-moduli cases as examples:

$$
\begin{aligned}
& \text { A: } \quad\left(\mathbb{P}^{4}[2,2,2,1,1] \| 8\right)_{-168}^{2}, \\
& \text { B: } \quad\left(\mathbb{P}^{5}[2,2,2,2,1,1] \| 4,6\right)_{-132}^{2}, \\
& \text { C: } \quad\left(\mathbb{P}^{6}[2,2,2,2,2,1,1] \| 4,4,4\right)_{-112}^{2} .
\end{aligned}
$$

They all have polynomial constraints of Fermat-type and exhibit the simplest singular locus, namely a singular curve with a $\mathbb{Z}_{2}$ action on the normal bundle induced from the ambient space, whose resolution gives as exceptional divisor a $\mathbb{p}^{1}$ bundle over that curve. For all complete intersections of Fermat type in a weighted space $\mathbb{P}^{n}[w]$ with $w_{n+1}=1$, we can define, in a generalization of (5.22), an $n$-dimensional pair of simplicial reflexive polyhedra $\Delta, \Delta^{*}$ as the convex hulls,

$$
\begin{aligned}
& \Delta=\mathrm{conv}\left(\begin{array}{l}
\nu_{1}=\left(\sum_{i} d_{i} / w_{1}-1,-1, \ldots,-1\right) \\
\vdots \\
\nu_{n}=\left(-1, \ldots,-1, \sum_{i} d_{i} / w_{n}-1\right) \\
\nu_{n+1}=(-1, \ldots \ldots, \ldots,-1)
\end{array}\right), \\
& \Delta^{*}=\mathrm{conv}\left(\begin{array}{l}
\nu_{1}^{*}=(1,0 \ldots, 0) \\
\vdots \\
\nu_{n}^{*}=(0, \ldots, 0,1) \\
\nu_{n+1}^{*}=\left(-w_{1}, \ldots,-w_{n}\right)
\end{array}\right),
\end{aligned}
$$

in an $n$-dimensional lattice. For all three examples we have, besides the origin $\nu_{0}^{*}=(0, \ldots, 0)$, exactly one additional point in $\Delta^{*}$, namely $\nu_{n+2}^{*}=(-1, \ldots,-1,0)$. Extending the lattice by $n-3$ dimensions, as described in Section 3, we find the linear relations among the extended lattice sites $\bar{\nu}_{i}^{*}$, which are summarized in the $l$-vectors,

$$
\begin{array}{ll}
\mathrm{A}: & l^{(1)}=(-4 ; 1,1,1,0,0,1), \\
& l^{(2)}=(0 ; 0,0,0,1,1,-2) \\
\mathrm{B}: & l^{(1)}=(-2,-3 ; 1,1,1,1,0,0,1), \\
& l^{(2)}=(0,0 ; 0,0,0,0,1,1,-2) ; \\
\mathrm{C}: & l^{(1)}=(-2,-2,-2 ; 1,1,1,1,1,0,0,1), \\
& l^{(2)}=(0,0,0 ; 0,0,0,0,0,1,1,-2) .
\end{array}
$$


(The above choices for the generators $l_{\mathrm{B}, \mathrm{C}}$ do not uniquely define the nef-partition of the lattice points of $\Delta^{*}$.) The associated GKZ system factorizes in all cases to a third and second order Picard-Fuchs equation, where the latter has the form

$$
L_{2}=\theta_{2}^{2}-z_{2}\left(2 \theta_{2}-\theta_{1}\right)\left(2 \theta_{2}-\theta_{1}+1\right) .
$$

Also the principal part of the third order operator is universal. We find

$$
\begin{array}{ll}
\text { A: } & L_{1}=\theta_{1}^{2}\left(2 \theta_{2}-\theta_{1}\right)-4 z_{1}\left(4 \theta_{1}+3\right)\left(4 \theta_{1}+2\right)\left(4 \theta_{1}+1\right) ; \\
\text { B: } & L_{1}=\theta_{1}^{2}\left(2 \theta_{2}-\theta_{1}\right)-6 z_{1}\left(2 \theta_{1}+1\right)\left(3 \theta_{1}+2\right)\left(3 \theta_{1}+1\right) ; \\
\text { C: } & L_{1}=\theta_{1}^{2}\left(2 \theta_{2}-\theta_{1}\right)-8 z_{1}\left(2 \theta_{1}+1\right)^{3}
\end{array}
$$

while the terms proportional to $z_{1}$ signal a different structure for the expansions of the solutions arround the singularity at $1 / z_{1}, 1 / z_{2} \rightarrow 0$, namely six pure power series solutions for $\mathrm{A}$ and $\mathrm{B}$ and solutions involving logarithms for $\mathrm{C}$. The Yukawa couplings

$$
\begin{aligned}
& K_{111}=\frac{1}{z_{1}^{3} \Delta_{0}}, \quad K_{112}=\frac{1-\kappa z_{1}}{z_{1}^{2} z_{2} \Delta_{0}}, \quad K_{122}=-\frac{1-2 \kappa z_{1}}{z_{1} z_{2} \Delta_{0} \Delta_{1}}, \\
& K_{222}=\frac{1-\kappa z_{1}+4 z_{2}-12 \kappa z_{1} z_{2}}{2 z_{2}^{2} \Delta_{0} \Delta_{1}^{2}}
\end{aligned}
$$

and the components of the discriminant $\Delta_{0}=\left(1-\kappa z_{1}\right)^{2}-4 \kappa^{2} z_{1}^{2} z_{2}, \Delta_{1}=1-4 z_{2}$ can be parametrized by $\kappa=256,108,64$ for the three cases in turn.

Using the model specific data (5.34)-(5.36) in our general formulas, we get the predictions of the topological data and the invariants of the rational curves listed in Table 5 up to degree three. In all cases we observe that $n_{0,1}^{\mathrm{r}}$ is the only nonvanishing invariant for $n_{0, i}^{\mathrm{r}}$, generally $n_{i, j}=0$ for $j>i$ and similar as in [15] $n_{i, j}^{\mathrm{r}}=n_{i, j-j}^{\mathrm{r}} \forall i>0, j \leqslant[i / 2]$. Furthermore we obtain that $n_{i}^{\mathrm{r}}=\sum_{j} n_{i, j}^{\mathrm{r}}$, where the $n_{i}^{\mathrm{r}}$ are the invariants for the rationals curves of the models $\left(\mathbb{P}^{5} \| 4,2\right)_{-176}^{1}$, $\left(\mathbb{P}^{6} \| 3,2,2\right)_{-144}^{1}$ and $\left(\mathbb{P}^{7} \| 2,2,2,2\right)_{-120}^{1}$ and the $n_{i, j}^{\mathrm{r}}$ are the ones for $\mathrm{A}, \mathrm{B}$ and $\mathrm{C}$, respectively. The invariants of the elliptic curves will be evaluated in Section 7. As before, we have checked that the topological numbers coincide after the change of basis with the ones calculated in [25].

In the general case the Picard-Fuchs equation will not follow as easy as above by factorization of the GKZ system. Rather, the analysis of additional symmetries of the period will be necessary, similar to as it is described for hypersurfaces in [16]. On the other hand the examples indicate that our description of the instanton

Table 5

\begin{tabular}{llllllllllll}
\hline Model & $x$ & $\int c_{2} J_{1}$ & $\int c_{2} J_{2}$ & \multicolumn{1}{c}{$K_{111}^{0}$} & $K_{112}^{0}$ & $n_{1,0}^{\mathrm{r}}$ & $n_{2,0}^{\mathrm{r}}$ & \multicolumn{1}{c}{$n_{2,1}^{\mathrm{r}}$} & \multicolumn{1}{c}{$n_{3,0}^{\mathrm{r}}$} & $n_{3,1}^{\mathrm{r}}$ & $n_{0,1}^{\mathrm{r}}$ \\
\hline A & -168 & 56 & 24 & 8 & 4 & 640 & 10032 & 72224 & 288384 & 7539200 & 4 \\
B & -132 & 60 & 24 & 12 & 6 & 360 & 2682 & 17064 & 35472 & 770280 & 6 \\
C & -112 & 64 & 24 & 16 & 8 & 256 & 1248 & 7232 & 10496 & 197632 & 8 \\
\hline
\end{tabular}


corrected Yukawa couplings also apply to the rich class of complete intersections with Gorenstein singularities.

The higher degree invariants for all complete intersections in products of weighted projective spaces and for all other examples discussed in this section can be evaluated by the programm INSTANTON.

\section{Connection with rational superconformal theories}

In this section we like to comment on different realisations of equivalent manifolds and their relation to exactly solvable superconformal theories. The sigma model on a Calabi-Yau manifold can be identified with a $(2,2)$ superconformal two-dimensional field theory, whose partition function and correlation functions are sometimes known exactly, at least at a special point in moduli space. Although more general identifications should exist, in the known examples the SCFT is a GSO projected tensor product of minimal $(2,2)$ superconformal field theories [52]. The classification of the latter follows an ADE pattern and there is a one-to-one correspondence to the classification of modality-zero ADE singularities. The defining equation of the latter can be viewed as Landau-Ginzburg potentials for a two-dimensional $(2,2)$ supersymmetric field theory having the SCFT as its infrared limit. The partition functions [53] of the ADE superconformal models at level $k$, as well as their coupings, are explicitly known [54]. The identification of their LG potentials is as follows:

$$
\begin{aligned}
& \mathrm{A}_{k} \sim z^{k+2}, \quad k \in \mathbb{Z}, \\
& \mathrm{D}_{k} \sim z^{k+2 / 2}+z y^{2}, \quad k \in 2 \mathbb{Z}, \\
& \mathrm{E}_{6} \sim y^{3}+z^{4}, \quad k=10, \\
& \mathrm{E}_{7} \sim z^{3}+z y^{3}, \quad k=16, \\
& \mathrm{E}_{8} \sim y^{3}+z^{5}, \quad k=28,
\end{aligned}
$$

and for tensor product models the LG potential is simply the sum of the corresponding LG potential terms. The central charge $c=\sum_{i=1}^{n} 3 k_{i} /\left(k_{i}+2\right)$ is the sum of the central charges of the factor theories and has to be nine to cancel the conformal anomaly.

In [55] a large number of identifications between GSO projected partition functions was found, among them $G_{1} \equiv\left(A_{2}, A_{2}, A_{2}, A_{6}, A_{6}\right) \simeq G_{2} \equiv$ $\left(A_{2}, A_{2}, A_{2}, D_{6}, D_{6}\right)$. We will argue that this implies an identification of the full string theory at a special point in the moduli space of the hypersurface $\mathrm{X}_{1}=$ $\left(\mathbb{P}^{4}[2,2,2,1,1] \| 8\right)_{-168}^{2}$ and of the complete intersection $\left.X_{2}=\left(\mathbb{p o p}^{\mathbb{P} 4} \|_{0}^{4}\right)_{-168}^{4}\right)^{2}$.

To see this, one has to perform a geometrical analog of the GSO projection in the tensor product theory on the LG model. One can either apply an heuristic path integral argument due to [56,55], or gauge the LG model as proposed in [57]. Both operations involve, similar to the GSO projection in the tensor product model, an orbifoldisation and one has to be careful to end up with the same symmetry group 
Table 6

\begin{tabular}{lrlr}
\hline & \multicolumn{1}{c}{$\mathrm{X}_{1}$} & $\mathrm{X}_{2}$ \\
\hline$K_{J J J}$ & 8 & $K_{J_{1} J_{1} J_{1}}$ & 8 \\
$K_{J D D}$ & 0 & $K_{J_{1} J_{1} J_{2}}$ & 4 \\
$K_{J D D}$ & -8 & $K_{J_{1} J_{2} J_{2}}$ & 0 \\
$K_{D D D}$ & -16 & $K_{J_{2} J_{2} J_{2}}$ & 0 \\
$\int c_{2} h_{J}$ & 56 & $\int c_{2} h_{J_{1}}$ & 56 \\
$\int c_{2} h_{D}$ & 8 & $\int c_{2} h_{J_{2}}$ & 24 \\
\hline
\end{tabular}

as in the SCFT. Before the GSO projection one has in the SCFT a $\mathbb{Z}_{k+2}$ symmetry in each factor model for the $\mathrm{A}_{k}$ theories and for the $\mathrm{D}_{k}$ theories if $k \in 4 \mathbb{Z}+2$. These symmetries are readily identified with the symmetries generated by $z \mapsto$ $\exp [2 \pi i z /(k+2)]$ and $z \mapsto \exp [4 \pi i z /(k+2)], y \mapsto \exp [-2 \pi i y /(k+2)]$ on the LG fields of the $A_{k}$ and $D_{k}$ models, respectively. The GSO projection on the tensor product theory is implemented by orbifoldisation with respect to the diagonal subgroup which in the above case is a $\mathbb{Z}_{\operatorname{lcm}\left\{k_{i}+2\right\}}$. The symmetry group of the GSO projected theory will therefore be $\mathscr{S} \times \prod_{i-1}^{5} \mathbb{Z}_{k_{i}+2} / \mathbb{Z}_{\operatorname{lcm}\left\{k_{i}+2\right\}}$, where $\mathscr{S}$ is permutation of identical factors. In the first argument [56] the orbifoldisation is replaced by a map of the variables $z_{i} \rightarrow \xi_{i}$ with constant jacobian such that the LG potential becomes linear in one or more of the $\xi_{i}$. They can be viewed as Lagrange multipliers, and integrating them out restricts the field configuration to an affine patch of a product of weighted projective spaces. For the SCFT of type $\mathrm{G}_{1}$ we have $z_{1}=\xi_{1}^{1 /\left(k_{1}+2\right)}, z_{i}=\xi_{i} \xi^{1 /\left(k_{i}+2\right)}, i=2, \ldots, 5$, with $|\partial \xi / \partial x|=$ const., precisely because $c=9$ implies $\sum_{i=1}^{5}\left[1 /\left(k_{i}+2\right)\right]=1$. Integrating out $\xi_{1}$ and going back to homogenous variables yields manifolds of type $X_{1}$, i.e. hypersurfaces in $\mathbb{P}^{4}[w]$. The diagonal subgroup of the phase symmetries on the $z_{i}$ is now trivial in $\mathbb{P}^{4}[\boldsymbol{w}]$, so that we end up with the same symmetry group as in the SCFT. Similarly, for the second type $\left(\mathrm{G}_{2}\right)$ we have $z_{1}=\xi_{1}^{1 /\left(k_{1}+2\right)}, z_{i}=\xi_{i} \xi_{1}^{1 /\left(k_{i}+2\right)}, i=2,3,4,6, \quad z_{5}=$ $\xi_{5}^{1 / 2} / \xi_{1}^{1 / 2\left(k_{1}+2\right)}, z_{7}=\xi_{7} \xi_{5}^{1 / 2} / \xi_{1}^{1 / 2\left(k_{1}+2\right)}$, and integrating out $\xi_{1}, \xi_{5}$ yields manifolds ${ }^{15}$ of type $\mathrm{X}_{2}$. The identification in $\mathbb{P}^{4}$ and $\mathbb{P}^{1}$ trivializes a $\mathbb{Z}_{8}$, so that the remaining symmetry is again as in the GSO projected SCFT. By the same combinatorics it is possible to introduce one or two gauge group operations respectively, which leave the superpotential invariant and lead, by the argument of [57], to the same geometrical interpretation.

Using the basis of divisors $J, D$ for the singular hypersurface described in [16] and the explicit formulas given there, we can calculate the intersection numbers in this basis. The evaluation of the second Chern form on $J$ is given in formula (2.6). These data and their analogs for the complete intersection, calculated by (2.5), are displayed in Table 6.

\footnotetext{
${ }^{15}$ We can interprete the LG potential $W=\sum_{i=1}^{5} x_{i}^{4}+x_{4} x_{6}^{2}+x_{5} x_{7}^{2}=0$ also as a four-dimensional hypersurface in the five-dimensional ambient space $\mathbb{P}^{5}(2,2,2,2,3,3)$ with $c_{1}=8$ and consider as in [58] a restricted cohomology of this space to define the six periods, which leads to the same prepotential and hence the same physical theory.
} 
The theorem of Wall, applicable for manifolds without torsion, states that $X_{1}$ and $X_{2}$ are homotopically equivalent if these topological numbers coincide, up to a linear transformation of the basis. Identifying $J_{1}=J$ and $J_{2}=\frac{1}{2}(J-D)$, we see that this is in fact the case. Model $X_{1}$ has been treated in great detail in $[16,15]$. In fact, one can prove that $\mathrm{X}_{1}$ and $\mathrm{X}_{2}$ are diffeomorphically equivalent, by realising both as singular fiber spaces where the generic fiber is a $\mathrm{K}_{3}$ over $\mathbb{P}^{1}$ with pairs of points identified on the latter. Similary we can find for the complete intersections $\mathrm{B}$ and $\mathrm{C}$ in one singular projective space, which were discussed in Section 5.8, diffeomorphic realisations in products of nonsigular projective spaces. We have the following equivalences:

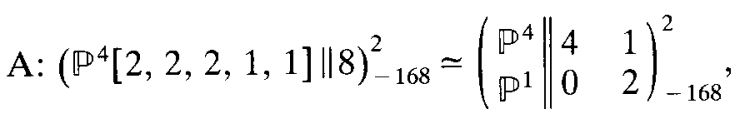

$$
\begin{aligned}
& \text { B: }\left(\mathbb{P}^{5}[2,2,2,2,1,1] \| 4,6\right)_{-132}^{2} \simeq\left(\begin{array}{c||ccc}
\mathbb{P}^{5} & 2 & 3 & 1 \\
\mathbb{P}^{1} & 0 & 0 & 2
\end{array}\right)_{-132}^{2} \text {, } \\
& C:\left(\mathbb{P}^{6}[2,2,2,2,2,1,1] \| 4,4,4\right)_{-112}^{2} \simeq\left(\begin{array}{l||llll}
\mathbb{P}^{6} & 2 & 2 & 2 & 1 \\
\mathbb{P}^{1} & 0 & 0 & 0 & 2
\end{array}\right)_{-112}^{2} \text {. }
\end{aligned}
$$

Let us discuss the solution for the model $\mathrm{X}_{2}$. Here we have the vertices of the dual polyhedra $\nu_{1,1}^{*}=(1,0,0,0 ; 0), \nu_{1,2}^{*}=(0,1,0,0 ; 0), \nu_{1,3}^{*}=(0,0,1,0 ; 0), \nu_{1,4}^{*}=$ $(0,0,0,1 ; 0), \nu_{1,5}^{*}=(-1,-1,-1,-1 ; 0)$ and $\nu_{2,1}^{*}=(0,0,0,0 ; 1), \nu_{2,2}^{*}=$ $(0,0,0,0 ;-1)$. We group them into two sets: $\mathrm{E}_{1}=\left\{\nu_{1,1}^{*}, \nu_{1,2}^{*}, \nu_{1,3}^{*}, \nu_{1,4}^{*}\right\}$ and $\mathrm{E}_{2}=$ $\left\{\nu_{1,5}^{*}, \nu_{2,1}^{*}, \nu_{2,2}^{*}\right\}$ and proceed as described in Section 3. This leads to

$$
l^{(1)}=(-4,-1 ; 1,1,1,1,1,0,0), \quad l^{(2)}=(0,-2 ; 0,0,0,0,0,1,1) .
$$

We can also write down the Laurent polynomials. They are

$$
\begin{aligned}
& P_{1}=a_{0}-a_{1} X_{1}-a_{2} X_{2}-a_{3} X_{3}-a_{4} X_{4}, \\
& P_{2}=b_{0}-\frac{b_{1}}{X_{1} X_{2} X_{3} X_{4}}-b_{2} Y_{1}-\frac{b_{3}}{Y_{1}} .
\end{aligned}
$$

Note that due to the freedom to rescale all variables and each polynomial, there are only two relevant parameters in $P_{1}$ and $P_{2}$, corresponding to the two complex structure moduli on $\mathrm{X}_{2}^{*}$. The period (3.7) follows straightforwardly by performing the integral (3.5). One also finds $z_{1}=a_{1} a_{2} a_{3} a_{4} b_{1} / a_{0}^{4} b_{0}$ and $z_{2}=b_{2} b_{3} / b_{0}^{2} . l^{(1)}$ and $l^{(2)}$ lead, after trivial factorization, to differential operators of orders four $\left(\mathscr{L}_{1}\right)$ and two $\left(\mathscr{L}_{2}=L_{2}\right)$, respectively. A third order operator $L_{1}$ can be obtained via $\mathscr{L}_{1}-4 \theta_{1}^{2} \mathscr{L}_{2}=\left(2 \theta_{2}+\theta_{1}\right) L_{1}$. The Yukawa couplings for the model $\mathrm{X}_{2}$ are found to be

$$
\begin{aligned}
K^{(3,0)} & =\frac{1}{z_{1}^{3} \Delta_{0}}, \quad K^{(2,1)}=\frac{1-256 z_{1}-4 z_{2}}{2 z_{1}^{2} z_{2} \Delta_{0} \Delta_{1}}, \quad K^{(1,2)}=\frac{3-512 z_{1}+4 z_{2} z_{1} z_{2}}{\Delta_{0} \Delta_{1}^{2}}, \\
K^{(0,3)} & =\frac{1-256 z_{1}+24 z_{2}-3072 z_{1} z_{2}+16 z_{2}^{2}}{2 z_{2}^{2} \Delta_{0} \Delta_{1}^{3}}
\end{aligned}
$$


with

$$
\Delta_{0}=\left(1-256 z_{1}\right)^{2}-4 z_{2}, \quad \Delta_{1}=\left(1-4 z_{2}\right) .
$$

The resulting topological invariants for the rational and elliptic curves for the cases $\mathrm{A}, \mathrm{B}$ and $\mathrm{C}$ in (6.2) are of course the same for both realisations. Model $\mathrm{X}_{1}$ was solved in $[16,15]$. In fact, in [15] the degrees of the curves are given with respect to the basis appropriate for $X_{2}$. We can find a map analogous to the one in (5.5) and hence a configuration $\left(\mathrm{Pp}^{1}\left\|_{0}^{4}\right\|_{2}^{4}\right)_{-168}^{2}$, which reproduces, via the integral (5.6), the expression for $w_{0}$. However, this configuration cannot be chosen to be transverse for generic points in the two-dimensional subspace of the moduli space under consideration. It is also interesting to note that the coordinates used here and the one used in $[16,15]$ are connected by a transcendental function.

A similar comparison can be made between the two models ( $\mathbb{P}^{4}[6,2,2$, 1 , 1] $\| 12)_{-252}^{2}$ and $\left(\mathrm{pl}^{\mathrm{P}}[3,1,1,1,1] \|_{0}^{6}{ }_{2}\right)_{-252}^{2}$. At special points in moduli space they correspond to the Gepner models $\left(A_{4}, A_{4}, A_{10}, A_{10}\right)$ and $\left(A_{4}, A_{4}, D_{10}, D_{10}\right)$, respectively. One finds again the relations $J_{1}=J$ and $J_{2}=\frac{1}{2}(J-D)$ between the divisors and identical topological invariants.

\section{Topological one-loop partition function and the number of elliptic curves}

Knowledge of the periods and the canonical coordinates allows, up to the difficulty of fixing a holomorphic ambiguity at each step, to recursively calculate higher loop topological partition funcions as was shown in [23]. To fix the ambiguity we need as gobal properties of the moduli space $\mathscr{M}$ the singularities discussed in Section 4.

We will focus on the one-loop case and calculate the expression defined in the $N=2$ SCFT on the torus as

$$
F_{1}=\frac{1}{2} \int \frac{\mathrm{d} \tau}{\tau_{2}} \operatorname{Tr}(-1)^{F} F_{\mathrm{L}} F_{\mathrm{R} q}^{L_{0}} \mathrm{q}^{\mathrm{L}_{0}},
$$

where the trace is to be taken over the left- and right-moving Ramond sectors. As shown in $[23,24]$ this quantity is, for the heterotic string with canonical embedding of the spin connection into the gauge group, related to the difference of the threshold corrections to the gauge couplings of the $\mathrm{E}_{6}$ and $\mathrm{E}_{8}$, namely $12 F_{1}=$ $\Delta\left(\mathrm{E}_{6}\right)-\Delta\left(\mathrm{E}_{8}\right)$. Using the holomorphic anomaly equation it was shown in [22] that it can be written as

$$
F_{1}=\log \left[M(z, \bar{z})|f(z)|^{2}\right],
$$

where the holomorphic-antiholomorphic mixing $M(z, \bar{z})$ is given by

$$
\log M=\sum_{p, q}(-1)^{p+q} \frac{p+q}{2} \operatorname{Tr}_{p, q} \log \operatorname{det}(g)-\frac{1}{12} \operatorname{Tr}(-1)^{F}
$$


here $g$ is the $t, \bar{t}$ metric introduced in [59]. It is related to the Weil-Peterson metric $G_{i j}$ by $g_{i j} / g_{0 \overline{0}}=g_{i j} \mathrm{e}^{K}=G_{i j}$ with $G_{i j}=\partial_{i} \partial_{j} K$. For $\sigma$-models on Calabi-Yau spaces (7.2) can be rewritten as

$$
F_{1}=\log \left[\exp \left(\left(3+k-\frac{1}{12} \chi\right) K\right) \operatorname{det}\left[G_{i j}\right]^{-1}|f(z)|^{2}\right] .
$$

In our application we will finally understand $F_{1}$ as a function of the Kähler moduli $t, \bar{t}$ which are related to $a, \bar{a}$ or $z, \bar{z}$ by the mirror map. To fix the holomorphic ambiguity $f(z)$ one considers the large volume limit $t, \bar{t} \rightarrow \infty$ for which one has the asymptotic behaviour

$$
\lim _{t, \bar{t} \rightarrow \infty} F_{1}=-\frac{2 \pi i}{12} \sum_{i=1}^{k}\left(t_{i}+\bar{t}_{i}\right) \int c_{2} J_{i}
$$

It was conjectured in [22] that $F_{1}^{\text {top }} \equiv \lim _{\bar{t} \rightarrow \infty} F_{1}$ has the following expansion:

$$
\begin{aligned}
F_{1}^{\text {top }}= & \text { const. }-\frac{2 \pi i}{12} \sum_{i=1}^{k} t_{i} \int c_{2} J_{i}-\sum_{n_{l}}\left\{2 n_{d_{1, \ldots}, d_{k}}^{\mathrm{e}} \log \left[\eta\left(\prod_{i=1}^{k} q_{i}^{d_{i}}\right)\right]\right. \\
& \left.+\frac{1}{6} n_{d_{1, \ldots, d_{k}} \mathrm{r}} \log \left(1-\prod_{i=1}^{k} q_{i}^{d_{i}}\right)\right\},
\end{aligned}
$$

in terms of the Euler numbers $n_{d_{1, \ldots}, d_{h}}^{\mathrm{r}}$ and $n_{d_{1, \ldots}, d_{k}}^{\mathrm{e}}$ of the tangentbundle over the appropriate compactified moduli space of the mappings from $\mathbb{P}^{1}$ and $\mathrm{T}^{2}$, respectively, to the Calabi-Yau space. In the case of isolated curves they count the number of rational curves and elliptic curves, respectively. Using (4.15), (4.12) one gets in the general case as the $\bar{t} \rightarrow \infty$ limit of (7.4)

$$
F_{1}^{\text {top }}=\log \left[\left(\frac{a_{1} \cdots a_{l}}{\omega_{0}}\right)^{3+k-\chi / 12} \frac{\partial\left(a_{1} \cdots a_{k}\right)}{\partial\left(t_{1} \cdots t_{k}\right)} f(z)\right]+\text { const.. }
$$

The factor $a_{1} \cdots a_{l} / \omega_{0}$ corresponds to the gauge choice with the fundamental period normalized as in (3.5) ${ }^{16}$. The holomorphic anomaly is determined by the requirement that $F_{1}^{\text {top }}$ has to be a regular function everywhere in moduli space except possibly at the components of the discriminate surface determined by $\Delta_{i}=0$, which can be directly determined from the Laurent polynomial (cf. Section (5.1). Besides the components $\Delta_{i}=0$ there appear also other singular loci $\delta_{i}=0$ in systems of Picard-Fuchs equations, which can be understood as identification singularities of the parameters space of the Laurent polynomial as it was discussed at the end of Section 3. In the cases we discuss we have $\delta_{i}=a_{i}$. While it is evident from (3.5) that $a_{1} \cdots a_{k} / \omega_{0}$ is regular at $\delta_{i}=0$, the jacobian of the mirror map (5.5) might have singularities at $\delta_{i}$. We therefore make the following general ansatz for $f(z)$ :

$$
f(z)=\Delta_{0}^{r_{0}} \cdots \Delta_{m}^{r_{m}} \delta_{1}^{s_{1}} \cdots \boldsymbol{\delta}_{k}^{s_{k}}
$$

\footnotetext{
${ }^{16} F_{1}^{\text {top }}$ depends of course on $k$ parameters $a_{i}, i=1, \ldots, k$. By the $\mathbb{C}^{*}$ symmetries, acting on the parameters of the Laurent polynomials, we can set $l-k$ of the $a_{i}$ appearing in (3.5) to one, if the number of polynomial constraints $l$ exceeds $k$.
} 
Table 7

\begin{tabular}{lccrrrrr}
\hline Model & $\chi$ & $\int c_{2} J$ & \multicolumn{1}{c}{$s_{1}$} & \multicolumn{1}{c}{$n_{1}^{\mathrm{e}}$} & \multicolumn{1}{c}{$n_{2}^{\mathrm{e}}$} & \multicolumn{1}{c}{$n_{3}^{\mathrm{e}}$} & \multicolumn{1}{c}{$n_{4}^{\mathrm{e}}$} \\
\hline $\mathrm{X}_{5}$ & -200 & 50 & 0 & 0 & 0 & 609250 & 37214316625 \\
$\mathrm{X}_{6}$ & -204 & 42 & 0 & 0 & 7884 & 145114704 & 1773044315001 \\
$\mathrm{X}_{8}$ & -296 & 44 & 1 & 0 & 41312 & 21464350592 & 1805292092664544 \\
$\mathrm{X}_{10}$ & -288 & 34 & 1 & 280 & 207680680 & 161279120326560 & 103038403740690105440 \\
\hline
\end{tabular}

Of course the $\Delta_{i}=z_{i}$ singularities are included in this ansatz. Inserting this ansatz in (7.7) and comparing the leading term with (7.5) yields equations for the $r_{i}$ and $s_{i}$. If the manifold happens to be transverse at $a=0$ the powers of the $\delta_{i}$, which in this case only have to compensate possible singularities of the jacobian, can in principle be determined by analytic continuation of the periods and hence the mirror map to the point $a=0$. In the general case we use the values the numbers $n_{d_{1, \ldots} d_{h}}^{\mathrm{e}}$ of a few elliptic curves of low poly-degree w.r.t. an integral basis of divisors, typically the fact that they have to vanish, to fix all parameters and predict the other numbers.

Let us first discuss as examples various one-moduli cases realized as hypersurfaces $\mathrm{X}_{k_{1}}$ of degree $k_{1}$ or complete intersections $\mathrm{X}_{k_{1}, \ldots, k_{n}}$ of multi-degree $k_{1, \ldots}, k_{n}$ in a single weighted projective space. From $[13,18,19]$ ' we have one component of the singular locus for all cases at $\Delta_{0}=\left(1-a^{\Sigma k_{i}}\right)$. We start with a short review of the one-moduli hypersurfaces in a weighted $\mathbb{P}^{4}$, denoted by $\mathrm{X}_{k}(w)$. In this case $\Delta_{0}$ is the only component of the singular locus, and it was observed in [23] that $r_{0}=-1 / 6$ for all cases yielding the invariants for the elliptic curves shown in Table 7.

Similarly for the one-moduli complete intersections in ordinary projective spaces (cf. [18]) we found $r_{0}=-1 / 6$ and the $s_{1}$ values indicated in Table 8. This result was obtained by requiring $n_{1}^{\mathrm{e}}=0$, which holds for intersections in ordinary projective spaces, and imposing (7.5). Note that these manifolds are not transverse at

Table 8

\begin{tabular}{lclllrrrrr}
\hline Model & $\chi$ & $f c_{2} J$ & \multicolumn{1}{l}{$s_{1}$} & $j$ & \multicolumn{1}{c}{1} & \multicolumn{1}{c}{2} & \multicolumn{1}{c}{3} & \multicolumn{1}{c}{4} \\
\hline $\mathrm{X}_{3,3}$ & -144 & 54 & 11 & $n_{j}^{\mathrm{r}}$ & 9 & 1053 & 52812 & 6424326 & 11394483834 \\
& & & & $n_{j}^{\mathrm{e}}$ & 0 & 0 & 3402 & 5520393 & 482074484 \\
$\mathrm{X}_{4,2}$ & -176 & 56 & $\frac{28}{3}$ & $n_{j}^{\mathrm{r}}$ & 8 & 1280 & 92288 & 15655168 & 3883902528 \\
& & & & $n_{j}^{\mathrm{e}}$ & 0 & 0 & 2560 & 17407072 & 24834612736 \\
$\mathrm{X}_{3,2,2}$ & -144 & \multirow{2}{*}{60} & $\frac{115}{6}$ & $n_{j}^{\mathrm{r}}$ & 12 & 720 & 22428 & 1611504 & 168199200 \\
& & & & $n_{j}^{\mathrm{e}}$ & 0 & 0 & 64 & 265113 & 198087264 \\
$\mathrm{X}_{2,2,2,2}$ & -128 & \multirow{2}{*}{64} & $\frac{85}{3}$ & $n_{j}^{\mathrm{r}}$ & 16 & 512 & 9728 & 416256 & 25703936 \\
& & & & $n_{j}^{\mathrm{e}}$ & 0 & 0 & 0 & 14752 & 8782848 \\
\hline
\end{tabular}


Table 9

\begin{tabular}{lccrrrrr}
\hline Model & \multicolumn{1}{c}{$\chi$} & \multicolumn{1}{c}{$c_{2} J$} & \multicolumn{1}{c}{$s_{1}$} & \multicolumn{1}{c}{$n_{1}^{\mathrm{e}}$} & \multicolumn{1}{c}{$n_{2}^{\mathrm{e}}$} & \multicolumn{1}{c}{$n_{3}^{\mathrm{e}}$} & \multicolumn{1}{c}{$n_{4}^{\mathrm{e}}$} \\
\hline $\mathrm{X}_{4,3}$ & -156 & 48 & $\frac{67}{6}$ & 0 & 27 & 16124238 & 38170438 \\
$\mathrm{X}_{6,2}$ & -256 & 52 & $\frac{29}{3}$ & 0 & -504 & 1228032 & 79275665304 \\
$\mathrm{X}_{4,4}$ & -144 & 40 & 11 & 0 & 1408 & 6953728 & 2684185380 \\
$\mathrm{X}_{6,4}$ & -156 & 32 & $\frac{31}{3}$ & 8 & 258336 & 5966034464 & 1267294361302800 \\
$\mathrm{X}_{6,6}$ & -120 & 22 & 9 & 360 & 40691736 & 4956204918240 & 616199133057629184 \\
\hline
\end{tabular}

$\Delta_{2}=a=0$. In order to compare with the results of Section 5.8 we list the rational curves for these models also in Table 8.

For the complete intesections in weighted projective spaces (cf. [19]) we found that $r_{0}=-1 / 6$ likewise and $s_{1}$ from (7.5) gives the integral expansion for the $n_{d}^{\mathrm{e}}$ as in Table 9. All invariants of the $X_{6,2}$ model are consistent with the indentification of this model with the one-parameter subspace of $\left(\mathbb{P}^{4}[6,2,2,1,1] \| 12\right)_{-252}^{2}$ [15], which implies $n_{i}^{\mathrm{e}}=\sum_{j} n_{i, j}^{\mathrm{e}}$. Especially $n_{2}^{\mathrm{e}}=-504$, which is the only negative invariant for a one-parameter family, is in agreement with $n_{2,0}^{\mathrm{e}}=n_{2,2}^{\mathrm{e}}=-492$, $n_{2,1}^{\mathrm{e}}=480$ for the two-parameter hypersurface (cf. [15]). We also checked that the identification of $X_{6,4}$ with the one-parameter subspace of $\left(\mathbb{P}^{4}[4,3,2,2,1] \| 12\right)^{2}$ [16] holds at the one-loop level. E.g. the lowest invariants of elliptic curves for the latter model are $n_{1,0}^{\mathrm{e}}=-2, n_{1,1}^{\mathrm{e}}=6$ and $n_{2,0}^{\mathrm{e}}=762, n_{2,1}^{\mathrm{e}}=-3060, n_{2,2}^{\mathrm{e}}=18918$, $n_{2,3}^{\mathrm{e}}=225096$ with the general symmetry $n_{i, j}=n_{i, 3 i-j}$ and $n_{i, j}=0$ for $j>3 i$; they reproduce the first two entries for the $X_{6,4}$ model above.

In summary the topological one-loop partition function for the one-parameter models is given by

$$
F_{1}^{\mathrm{top}}=\log \left[\frac{a^{\Sigma_{i} k_{i} / c_{2} J / 12}}{w_{0}^{4-(x+4) / 12}}\left(\frac{1}{a} \frac{\partial a}{\partial t}\right)^{1 / 2} K_{t t t}^{1 / 6}\right] .
$$

Next we treat a hypersurface $\mathrm{X}_{14}(7,2,2,2,1)$ with two moduli. The singular locus is, in addition to the lines $z_{1}=0, z_{2}=0$, given by

$$
\begin{aligned}
& \Delta_{0}=1+27 z_{1}-63 z_{1} z_{2}+56 z_{1} z_{2}^{2}-112 z_{1} z_{2}^{3}-\left(7-4 z_{2}\right)^{4} z_{1}^{2} z_{2}^{3}, \\
& \Delta_{1}=4 z_{2}-7 .
\end{aligned}
$$

Eq. (3.6) and the analog of (3.3) from [16], $l^{(1)}=(-7 ; 0,1,1,1,-3,-7)$ and $l^{(2)}=(0 ; 1,0,0,0,1,-2)$, define ${ }^{17}$ the relation $z_{1}=a_{2}^{7} / a_{1}^{7}$ and $z_{2}=7 / a_{2}^{2}$ by which we transform the expressions of singular components (7.10) to the $a_{i}$ variables. Beside these components we have to care about the sets $\delta_{1}=a_{1}=0$ and $\delta_{2}=a_{2}=0$. For the further calculation it turns out to be advantegous to get rid of the denominators in the transformed expressions (7.10) by rescaling $\Delta_{1} \rightarrow a^{14} \Delta_{1}$ and $\Delta_{2} \rightarrow b^{2} \Delta_{2}$.

\footnotetext{
${ }^{17}$ Note the scale factor 7 introduced in [16] in order to simplify (7.10).
} 
Table 10

\begin{tabular}{lrrrrrrrr}
\hline$j$ & \multicolumn{1}{c}{0} & 1 & 2 & 3 & 4 & 5 & 6 & 7 \\
\hline$n_{0, j}^{\mathrm{r}}$ & 0 & 28 & 0 & 0 & 0 & 0 & 0 & 0 \\
$n_{0, j}^{\mathrm{e}}$ & 0 & 0 & 0 & 0 & 0 & 0 & 0 & 0 \\
$n_{1, j}^{\mathrm{r}}$ & 3 & -56 & 378 & 14427 & 14427 & 378 & -56 & 3 \\
$n_{1, j}^{\mathrm{e}}$ & 0 & 0 & 0 & 0 & 0 & 0 & 0 & 0 \\
$n_{2, j}^{\mathrm{r}}$ & -6 & 140 & -1512 & 9828 & -69804 & 500724 & 29683962 & 68588248 \\
$n_{2, j}^{\mathrm{e}}$ & 0 & 0 & 0 & 0 & 0 & 378 & 6496 & 27564 \\
\hline
\end{tabular}

The Euler number is $\chi=-240$, the Hodge numbers are $h^{1,1}=2, h^{2,1}=122$. We calculate $\int c_{2} \wedge J_{1}=44$ and $\int c_{2} \wedge J_{2}=126$, where $J_{1}, J_{2}$ are the basis which generates $\mathrm{H}^{2}(\mathrm{X}, \mathbb{Z})$. It is connected with the $(1,1)$ forms dual to the natural basis of the divisors in the polyheder construction $J, D$ used in [16], by $J_{1}=J$ and $J_{2}=\frac{1}{2}(7 J-D)$. From (7.5) we get two equations,

$$
r_{0}=-\frac{s_{1}}{14}-\frac{1}{42}, \quad r_{1}=-\frac{s_{2}}{2}-\frac{17}{6}
$$

The vanishing of the numbers of curves $n_{0,1}^{\mathrm{e}}=s_{2}$ and $n_{1,0}^{\mathrm{e}}=\frac{27}{28}\left(2-s_{1}\right)$ enforces $s_{1}=2$ and $s_{2}=0$. Other numbers of elliptic curves $n_{i, j}^{\mathrm{e}}$ with $i<3$ are then given in Table 10, where we list for convenience also the number of rational curves $n_{i, j}^{\mathrm{r}}$.

Here we have a symmetry $n_{i, j}=n_{i, 7 i-j}$ and $n_{i, j}=0$ for $j>7 i$. The identification of the one-parameter subspace of this model via $n_{i}=\sum_{j} n_{i, j}$ with the hypersurface $\left(\mathbb{P}^{4}[1,1,1,1,4] \| 8\right)_{-256}^{1}$ observed in $[16]$ can also be checked at one-loop level.

Next we treat a hypersurface in a product of two projective spaces,

$$
\left(\begin{array}{l||l}
\mathbb{P}^{2} & 3 \\
\mathbb{P}^{2} & 3
\end{array}\right)_{-162}^{2}
$$

The number $n_{d 1, d_{2}}^{\mathrm{r}}$ of rational curves of bidegree $\left(d_{1}, d_{2}\right)$ was obtained in [16]. The evaluation of the second Chern class on $J_{1}, J_{2}$ can be read off from (2.5); $\int c_{2} \wedge J_{1}=\int c_{2} \wedge J_{2}=36$. Because of the symmetry, we have $s_{1}=s_{2}$. In this case we have to consider only one component,

$$
\Delta_{0}=1-\left(1-z_{1}\right)^{3}+\left(1-z_{2}\right)^{3}+3 z_{1} z_{2}\left(z_{1}+z_{2}+7\right),
$$

of the discriminant of the complete intersection, where the connection with the parameters $a_{1}, a_{2}$ is encoded in the $l^{(i)}$ given by (3.3), $z_{1}=3^{3} / a_{1}^{3}$ and $z_{2}=3^{3} / a_{2}^{3}$. Again after clearing the denominator, the comparison with the large radius limit gives us the relation $r_{0}=-\frac{7}{6}-\frac{1}{9} s_{1}$, leaving us with one unknown constant $s_{1}$, which is determined by requiring $n_{0,1}^{\mathrm{e}}=\frac{9}{2}\left(9+s_{1}\right)=0$. Table 11 contains the numbers of rational and elliptic curves up to bidegree $d_{1}+d_{2} \leqslant 6$. Because of the exchange symmetry we list only $n^{i}\left(d_{1}, d_{2}\right)$ with $d_{1} \leqslant d_{2}$. Here we observe $n_{1,3}^{\mathrm{e}}=$ $-2 n_{0,1}^{\mathrm{r}}, n_{2,2}^{\mathrm{e}}=n_{1,1}^{\mathrm{r}}$ and $n_{1, d}^{\mathrm{e}}=-2 n_{1, d_{2}-3}^{\mathrm{r}}$ for $d_{2} \geqslant 4$.

For the three-generation complete intersection case (Section 5.1) the relevant components of the discriminant surface were obtained by the different methods 
Table 11

\begin{tabular}{|c|c|c|c|c|c|c|c|c|}
\hline \multicolumn{3}{|c|}{$\left(d_{1}, d_{2}\right)$} & \multicolumn{3}{|c|}{$\left(d_{1}, d_{2}\right)$} & \multicolumn{3}{|c|}{$\left(d_{1}, d_{2}\right)$} \\
\hline \multirow[t]{2}{*}{$(0,1)$} & $n^{\mathrm{r}}$ & 189 & $(1,1)$ & $n^{\mathrm{r}}$ & 8262 & $(2,2)$ & $n^{\mathrm{r}}$ & 1310892 \\
\hline & $n^{\mathrm{e}}$ & 0 & & $n^{\mathrm{e}}$ & 0 & & $n^{\mathrm{e}}$ & 8262 \\
\hline \multirow[t]{2}{*}{$(0,2)$} & $n^{\mathrm{r}}$ & 189 & $(1,2)$ & $n^{\mathrm{r}}$ & 142884 & $(2,3)$ & $n^{\mathrm{r}}$ & 516953097 \\
\hline & $n^{\mathrm{e}}$ & 0 & & $n^{\mathrm{e}}$ & 0 & & $n^{\mathrm{e}}$ & 1519434 \\
\hline \multirow[t]{2}{*}{$(0,3)$} & $n^{\mathrm{r}}$ & 162 & $(1,3)$ & $n^{\mathrm{r}}$ & 1492290 & $(2,4)$ & $n^{\mathrm{r}}$ & 12289326723 \\
\hline & $n^{\mathrm{e}}$ & 3 & & $n^{\mathrm{e}}$ & -378 & & $n^{\mathrm{e}}$ & 71809416 \\
\hline \multirow{2}{*}{$(0,4)$} & $n^{\mathrm{r}}$ & 189 & $(1,4)$ & $n^{\mathrm{r}}$ & 11375073 & $(3,3)$ & $n^{\mathrm{r}}$ & 55962304650 \\
\hline & $n^{\mathrm{e}}$ & 0 & & $n^{\mathrm{e}}$ & -16524 & & $n^{\mathrm{e}}$ & 818388234 \\
\hline \multirow[t]{2}{*}{$(0,5)$} & $n^{\mathrm{r}}$ & 189 & $(1,5)$ & $n^{\mathrm{r}}$ & 69962130 & & & \\
\hline & $n^{\mathrm{e}}$ & 0 & & $n^{\mathrm{e}}$ & -285768 & & & \\
\hline \multirow[t]{2}{*}{$(0,6)$} & $n^{\mathrm{r}}$ & 162 & & & & & & \\
\hline & $n^{\mathrm{e}}$ & 0 & & & & & & \\
\hline
\end{tabular}

above: $\Delta_{0}=\left(1-27 z_{1}\right)^{3}-27 z_{2}, \Delta_{1}=1-27 z_{2}$. Proceeding as before we get $r_{0}=$ $-\frac{1}{6}-\frac{1}{9} s_{2}, r_{1}=3-s_{1}+s_{2}$, and the vanishing of $n_{1,0}^{\mathrm{e}}=\frac{9}{2} s_{2}$ requires $s_{2}=0$, and then from $n_{1,1}^{\mathrm{e}}=\frac{3}{2}\left(9 s_{1}-27\right)=0$ we get $s_{1}=3$. The corresponding predictions for $n_{d_{1}, d_{2}}^{\mathrm{e}}$ are shown in Table 12 .

For the Tian-Yau manifold example (Section 5.2) the discriminant can be read off from the Yukawa couplings $\Delta_{0}=1-27 z_{1}-27 z_{2}, \Delta_{1}=1-27 z_{1}, \Delta_{2}=1-27$

Table 12

\begin{tabular}{lrlr}
\hline$\left(d_{1}, d_{2}\right)$ & $n_{d_{1}, d_{2}}^{\mathrm{e}}$ & $\left(d_{1}, d_{2}\right)$ & \multicolumn{1}{c}{$n_{d_{1}, d_{2}}^{\mathrm{e}}$} \\
\hline$(1,0)$ & 0 & $(1,1)$ & 0 \\
$(2,0)$ & 0 & $(2,2)$ & -16028 \\
$(3,0)$ & 3 & $(3,3)$ & -124719 \\
$(4,0)$ & 0 & $(2,1)$ & 0 \\
$(5,0)$ & 0 & $(4,2)$ & -924372 \\
$(6,0)$ & 0 & $(3,1)$ & -126 \\
$(0,1)$ & 0 & $(4,1)$ & -1944 \\
$(0,2)$ & -27 & $(5,1)$ & -30618 \\
$(0,3)$ & 81 & $(1,2)$ & 972 \\
$(0,4)$ & -324 & $(2,4)$ & -426222 \\
$(0,5)$ & 1728 & $(3,2)$ & 159678 \\
$(0,6)$ & -8955 & $(1,3)$ & -486 \\
& & $(2,3)$ & 27945 \\
& & $(1,4)$ & 22356 \\
& & $(1,5)$ & -72900 \\
\hline
\end{tabular}


Table 13

\begin{tabular}{lrlrlr}
\hline$\left(d_{1}, d_{2}\right)$ & $n_{d_{1} d_{2}}^{\mathrm{e}}$ & $\left(d_{1}, d_{2}\right)$ & \multicolumn{1}{c}{$n_{d_{1} d_{2}}^{\mathrm{e}}$} & \multicolumn{1}{c}{$\left(d_{1}, d_{2}\right)$} & $n_{d_{1} d_{2}}^{\mathrm{e}}$ \\
\hline$(0,1)$ & 0 & $(1,1)$ & 0 & $(1,3)$ & 1458 \\
$(0,2)$ & -27 & $(2,2)$ & -2916 & & \\
$(0,3)$ & 81 & $(3,3)$ & 108180 & $(1,4)$ & 4374 \\
$(0,4)$ & -324 & $(1,2)$ & 324 & $(1,5)$ & 15066 \\
$(0,5)$ & 1728 & $(2,4)$ & -31104 & $(2,3)$ & -13176 \\
$(0,6)$ & -8955 & & & & \\
\hline
\end{tabular}

$z_{2}$. Obviously $r_{1}=r_{2}$ and $s_{1}=s_{2}$ and (7.5) yields $r_{0}=\frac{1}{2}+\frac{1}{3} s_{1}-r_{1}$. The predictions in Table 13 are obtained by imposing $n_{0,1}^{\mathrm{e}}=\frac{9}{2}\left(s_{1}-2\right)=0$ and $n_{1,1}^{\mathrm{e}}=\frac{243}{2}\left(3 r_{1}-4\right)=$ 0 . Note that invariants $n_{0, i}^{\mathrm{e}}$ of the example in Section 5.1 coincide with $n_{i, 0}^{\mathrm{e}}=n_{0, i}^{\mathrm{e}}$ of the present one.

The three-moduli example in Section 5.5 has the general discriminant

$$
\begin{aligned}
\Delta_{0}= & 1-108 z_{1}+4374 z_{1}^{2}-78732 z_{1}^{3}+531441 z_{1}^{4}-8 z_{2}+432 z_{1} z_{2}-5832 z_{1}^{2} z_{2} \\
& +16 z_{2}^{2}-8 z_{3}+432 z_{1} z_{3}-5832 z_{1}^{2} z_{3}-23 z_{2} z_{3}+16 z_{3}^{2}
\end{aligned}
$$

and a second component of the discriminant locus

$$
\Delta_{1}=1-8 z_{2}+16 z_{2}^{2}-8 z_{3}-32 z_{2} z_{3}+16 z_{3}^{2} \text {. }
$$

As a slight technical simplification we replace $a_{1} \ldots a_{k} / w_{0}$ by $1 / w_{0}$ in (7.7), set $\delta_{i}=z_{i}$ in (7.8) and work throughout with the large complex structure parameters $z_{i}$, which is possible as the $z_{i}$ are the good coordinates on $\mathscr{M}$ (cf. Section 3).

By (7.5) we can fix in this case $s_{1}=-4, s_{2}=s_{3}=-3$, and from $n_{0,0,1}^{\mathrm{e}}=-9(1+$ $\left.6 r_{0}\right)=0$ we have again $r_{0}=-1 / 6$. Enforcing also $n_{1,0,0}^{\mathrm{e}}=-4\left(r_{0}+r_{1}\right)=0$ we get the predictions in Table 14 for the non-zero invariants of the elliptic curves up to

\begin{tabular}{|c|c|c|c|c|c|}
\hline$\left(d_{1}, d_{2}, d_{3}\right)$ & $n_{d_{1}, d_{2}, d_{3}}^{\mathrm{e}}$ & $\left(d_{1}, d_{2}, d_{3}\right)$ & $n_{d_{1}, d_{2}, d_{3}}^{\mathrm{e}}$ & $\left(d_{1}, d_{2}, d_{3}\right)$ & $n_{d_{1}, d_{2}, d_{3}}^{\mathrm{e}}$ \\
\hline$(0,1,1)$ & -12 & $(2,1,3)$ & -3024 & $(4,0,1)$ & -432 \\
\hline$(0,2,2)$ & 15 & $(2,2,3)$ & -2052 & $(4,0,2)$ & -4212 \\
\hline $\begin{array}{l}(1,1,1) \\
(2,2,2)\end{array}$ & $\begin{array}{r}288 \\
-13284\end{array}$ & $(3,0,0)$ & 4 & $(4,0,3)$ & -432 \\
\hline$(1,1,2)$ & 2888 & $(3,0,1)$ & -36 & $(4,1,1)$ & -464296 \\
\hline$(1,2,2)$ & 2160 & $(3,0,2)$ & -36 & $(4,1,2)$ & -330300 \\
\hline$(1,2,3)$ & 2160 & $(3,0,3)$ & 85212 & $(5,0,1)$ & -4212 \\
\hline$(1,3,3)$ & 11664 & $(3,1,1)$ & 17580 & $(5,0,2)$ & -191484 \\
\hline$(2,1,1)$ & -3024 & $(3,1,2)$ & 48024 & $(5,1,1)$ & -46008 \\
\hline \multirow[t]{2}{*}{$(2,1,2)$} & -4320 & $(3,1,3)$ & 48024 & $(6,0,1)$ & -35820 \\
\hline & & $(3,2,2)$ & 991536 & & \\
\hline
\end{tabular}
bidegree seven.

Table 14 
Table 15

\begin{tabular}{lllllrrr}
\hline Model & $k$ & $r_{k}$ & $s_{k+1}$ & $j$ & \multicolumn{1}{c}{0} & 1 & \multicolumn{2}{c}{} \\
\hline A & 0 & $-\frac{1}{6}$ & $-\frac{17}{6}$ & $n_{i<3, j}^{\mathrm{e}}$ & 0 & 0 & 0 \\
& 1 & $-\frac{5}{6}$ & -3 & $n_{3, j}^{\mathrm{e}}$ & -1280 & 2560 & 2560 \\
& & & & $n_{4, j}^{\mathrm{e}}$ & -317864 & 1047280 & 15948240 \\
$\mathrm{~B}$ & 0 & $-\frac{1}{6}$ & -6 & $n_{i<2, j}^{\mathrm{e}}$ & 0 & 0 & 0 \\
& 1 & -1 & -3 & $n_{2, j}^{\mathrm{e}}$ & -16 & 48 & -16 \\
& & & & $n_{3, j}^{\mathrm{e}}$ & -5364 & 18972 & 237897 \\
$\mathrm{C}$ & 0 & $-\frac{1}{6}$ & $-\frac{19}{6}$ & $n_{i<4, j}^{\mathrm{e}}$ & 0 & 0 & 0 \\
& 1 & $-\frac{7}{6}$ & -3 & $n_{4, j}^{\mathrm{e}}$ & -280 & 1120 & 13072 \\
& & & & $n_{5, j}^{\mathrm{e}}$ & -20992 & 119808 & 429608 \\
\hline
\end{tabular}

We finally evaluate in Table 15 the invariants of the elliptic curves for the hypersurface and the complete intersections in one singular projective space discussed in Section 5.8. The topological data can be found in Table 5, and the discriminant may be read of from (5.37). The invariants for case $\mathrm{A}$ are in accordance with [15]. Also for the complete intersections we obtain for the invariants $n_{i, j}^{\mathrm{e}}=0$ for $j>i$ as well as the symmetry $n_{i, j}^{\mathrm{e}}=n_{i, j-i}^{\mathrm{e}}$. Comparison with the models $\left(\mathbb{P}^{5} \| 4,2\right)_{-176}^{1},\left(\mathbb{P}^{6} \| 3,2,2\right)_{-144}^{1}$ and $\left(\mathbb{P}^{7} \| 2,2,2,2\right)_{-120}^{1}$ reveals the expected relation $n_{i}^{\mathrm{e}}=\sum_{j} n_{i, j}^{\mathrm{e}}$, which is an affirmative consistency check of the calculations at one-loop level.

In all our examples we observed that the holomorphic anomaly is of index $r_{0}=-1 / 6$ at the general component of the discriminant. This also holds true for examples with higher dimensional moduli spaces and for models with a somewhat different type of singularity such as $\left(\mathbb{P}^{4}[9,6,1,1,1] \| 18\right)_{-540}^{2}$ or $\left(\mathbb{P}^{4}[12,8,2,1\right.$, 1] $\| 24)_{-480}^{3}$. This is related to the fact that the manifolds always approach a nodal configuration along this component of the discriminant. The exponent of the holomorphic ambiguity seems to be universal for this type of singularity.

\section{Discussion}

To extend the discussion of mirror symmetry to CICY manifolds with higher dimensional moduli spaces, we have described how to set up the Picard-Fuchs equations and specified the point of maximal unipotent monodromy. We have developed a convenient way to construct all its solutions around this point by showing the equilvalence of the solutions with the elements of special representatitves of the ring $\mathscr{R}$. In fact, the top element of $\mathscr{R}$ corresponds to the cubic monomials of intersection numbers for the generating elements of $\mathrm{H}^{2}(\hat{\mathrm{X}}, \mathbb{Z})$. We have found very simple formulas for the instanton corrected intersection numbers from which the number of rational curves can be obtained, always assuming that mirror symmetry is correct. In this paper we have focussed on the region of the moduli space of large Kähler and complex structure. The extension to the whole 
moduli space requires analytical continuation of the periods. This is in principle straightforward using an integral representation of the solutions of the PicardFuchs equations.

Technically, this is, however, rather involved and was performed so far only for one-moduli cases [11,13,14] and for two types of two-moduli models [15].

The form of the topological partition function is fixed by the holomorphic anomaly equation up to a holomorphic function. To specify the latter we had to analyse their asymptotic behaviour at the singular locus of the Picard-Fuchs system. In general we had to use the vanishing of the elliptic curves of low degree to provide this information.

From a more technical point of view the method described here requires, for the prediction of the instanton expansion in the large Kähler structure limit, only the generators of the Mori cone $l^{(i)}$ and the associated intersection numbers. Given these data, the expansion for the corrected Yukawa couplings and the prepotential can be simply obtained via (4.18), (4.19). As it bypasses the evaluation of the Yukawa couplings on the complex structure side, it is applicable to higher dimensional moduli spaces where the evaluation of these complicated algebraic expressions is extremely tedious. It should be clear from Sections 5.7 and 5.8 that the data mentioned above can be provided more generally for the moduli associated to algebraic deformations in the general class of Calabi-Yau manifolds representable as hypersurfaces or complete intersections in toric varieties ${ }^{18}$. It would be interesting to see if these data can also be obtained for the twisted sectors of the models in the above class and for those of the 3345 Landau-Ginzburg models with more then five fields constructed in [62], which cannot be reduced to CY threefolds as the examples in Section 6 and others in [55], but have only an interpretation as higher dimensional manifolds with $c_{1}>0$.

The calculation of the prepotential was discussed. This problem can also be considered from the point of view of topological field theory. In fact in many cases the prepotential can be obtained from the axioms of topological field theory contained in the Witten-Dijkgraaf-Verlinde-Verlinde equations [63]. However, for this approach the Calabi-Yau manifolds are a critical case because here the operator algebra of topological field theory is nilpotent, at least if we restrict ourself to the massless perturbations. For the threefolds the information from the WDVV equations just defines special geometry, but does not give further information on the prepotential.

From the identification of the two hypersurfaces $\left(\mathbb{P}^{4}[2,2,2,1,1] \| 8\right)_{-168}^{2}$ and $\left(\mathbb{P}^{4}[6,2,2,1,1] \| 12\right)_{-252}^{2}$ with complete intersections, which was also confirmed by comparison with the Gepner models, we are taught to view the system $L_{1}, \ldots, L_{k}$, which contains (at the point $z=0$ ) the information about the ring $\mathscr{R}$, the object of primary interest.

\footnotetext{
${ }^{18}$ In fact, the methods can be easily extended to evaluate three-point functions on higher dimensional Calabi-Yau spaces with higher dimensional moduli spaces [60]. Some results for one-parameter families were obtained in [61].
} 
Going one step further back one may see as the basic input the data of a Riemann-Hilbert problem with sympletic integral representations of the monodromy and singular points, where the solutions are characterised by a graded ring whose homogeneous sub-spaces are of suitable type. In fact these data seem to encode all topological data required for the classification of the homotopy type of families of Calabi-Yau manifolds by the theorem of Wall and might lead to a refined classification of $N=2$ vacua of string theory and Calabi-Yau threefolds.

\section{Acknowledgements}

We would like to thank V. Batyrev, M. Berschadsky, B. Lian, J. Louis, S.S. Roan and A. Todorov for discussions.

\section{Appendix A}

The pole structure in the coefficients of the logarithmic solutions to the Picard-Fuchs equation

In this appendix we exhibit the pole structures of the logarithmic solutions of the Picard-Fuchs equations.

The starting point are the generators of the Mori cone $l^{(\alpha)}$ which are all of the form 19

$$
\begin{aligned}
l^{(\alpha)} & =\left(-\left\{l_{0_{j}}^{(\alpha)}\right\},\left\{l_{i}^{(\alpha)}\right\}\right), \\
& j=1, \ldots, \text { number of polynomials }, \quad \alpha=1, \ldots, h^{1,1},
\end{aligned}
$$

where

$$
-\sum_{j} l_{0_{j}}^{(\alpha)}+\sum_{i} l_{i}^{(\alpha)}=0, \quad l_{i}^{(\alpha)} \in \mathbb{Z}, \quad l_{0_{j}}^{(\alpha)} \in \mathbb{Z} \geqslant .
$$

The fundamental period is

$$
w_{0}(z)=\sum_{n} c(n) z^{n}
$$

where the sum is over all non-negative integers $n_{\alpha}$ and the expansion coefficients are

$$
c(n)=\frac{\prod_{j}\left(\sum_{\alpha} l_{0_{j}}^{(\alpha)} n_{\alpha}\right) !}{\prod_{i}\left(\sum_{\alpha} l_{i}^{(\alpha)} n_{\alpha}\right) !}
$$

\footnotetext{
${ }^{19}$ We have changed the sign of the components $l_{0_{j}}^{(\alpha)}$ as compared to (3.3) for notational convenience.
} 
We then define

$$
w_{0}(z, \rho)=\sum_{n} c(n, \rho) z^{n+\rho}
$$

with

$$
c(n, \rho)=\frac{\prod_{j} \Gamma\left[\Sigma_{\alpha} l_{0_{j}}^{(\alpha)}\left(n_{\alpha}+\rho_{\alpha}\right)+1\right]}{\prod_{i} \Gamma\left[\Sigma_{\alpha} l_{i}^{(\alpha)}\left(n_{\alpha}+\rho_{\alpha}\right)+1\right]} .
$$

The logarithmic solutions contain the coefficients

$$
\begin{aligned}
& \text { (I) }\left.\partial_{\beta} c(n) \equiv \frac{\partial}{\partial_{\beta}} c(n+\rho)\right|_{\rho=0}, \\
& \text { (II) } \partial_{\beta} \partial_{\gamma} c(n) \\
& \text { (III) } \partial_{\beta} \partial_{\gamma} \partial_{\delta} c(n) .
\end{aligned}
$$

The following definitions will become useful:

$$
\begin{aligned}
& A^{(\beta)}(n)=\sum_{j} l_{0_{j}}^{(\beta)} \psi\left(\sum_{\alpha} l_{0_{j}}^{(\alpha)} n_{\alpha}+1\right)-\sum_{i} l_{i}^{(\beta)} \psi\left(\sum_{\alpha} l_{i}^{(\alpha)} n_{\alpha}+1\right), \\
& B^{(\beta \gamma)}(n)=\sum_{j} l_{0_{j}}^{(\beta)} l_{0_{j}}^{(\gamma)} \psi^{\prime}\left(\sum_{\alpha} l_{0_{j}}^{(\alpha)} n_{\alpha}+1\right)-\sum_{i} l_{i}^{(\beta)} l_{i}^{(\gamma)} \psi^{\prime}\left(\sum_{\alpha} l_{i}^{(\alpha)} n_{\alpha}+1\right), \\
& C^{(\beta \gamma \delta)}(n)=\sum_{j} l_{0_{j}}^{(\beta)} l_{0_{j}}^{(\gamma)} l_{0_{j}}^{(\delta)} \psi^{\prime \prime}\left(\sum_{\alpha} l_{0_{j}}^{(\alpha)} n_{\alpha}+1\right)-\sum_{i} l_{i}^{(\beta)} l_{i}^{(\gamma)} l_{i}^{(\gamma)} \psi^{\prime \prime}\left(\sum_{\alpha} l_{i}^{(\alpha)} n_{\alpha}+1\right),
\end{aligned}
$$

where $\psi$ is the logarithmic derivative of the Gamma function. We then get the expressions

$$
\begin{aligned}
& \partial_{\beta} c(n)=c(n) A^{(\beta)}(n), \\
& \partial_{\beta} \partial_{\gamma} c(n)=c(n)\left[A^{(\beta)}(n) A^{(\gamma)}(n)+B^{(\beta \gamma)}(n)\right], \\
& \partial_{\beta} \partial_{\gamma} \partial_{\delta} c(n)=c(n)\left[A^{(\beta)}(n) A^{(\gamma)}(n) A^{(\delta)}(n)+A^{(\beta)}(n) B^{(\gamma \delta)}(n)\right. \\
& \\
& \left.+A^{(\gamma)}(n) B^{(\delta \beta)}(n)+A^{(\delta)}(n) B^{(\beta \gamma)}(n)+C^{(\beta \gamma \delta)}(n)\right],
\end{aligned}
$$

whose pole structures we have to examine. Before doing this we define

$$
A_{k_{1}, \ldots, k_{p}}^{(\beta)}(n)=\sum_{j} l_{0_{j}}^{(\beta)} \psi\left(\sum_{\alpha} l_{0_{j}}^{(\alpha)} n_{\alpha}+1\right)-\sum_{i \neq k_{1}, \ldots, k_{p}} l_{i}^{(\beta)} \psi\left(\sum_{\alpha} l_{i}^{(\alpha)} n_{\alpha}+1\right),
$$

and likewise for $B_{k_{1}, \ldots, k_{p}}^{(\beta \gamma)}(n)$ and $C_{k_{1}, \ldots, k_{p}}^{(\beta \gamma \delta)}(n)$. For $\sum_{\alpha} l_{k}^{(\alpha)} n_{\alpha}<0$ we set

$$
\sum_{\alpha} l_{k}^{(\alpha)} n_{\alpha} \equiv-m_{k}
$$


and

$$
\begin{aligned}
& \tilde{A}_{k_{1}, \ldots, k_{p}}^{(\beta)}=A_{k_{1}, \ldots, k_{p}}^{(\beta)}-\sum_{i=1}^{p} l_{k_{i}}^{(\beta)} \psi\left(m_{k_{i}}\right), \\
& \tilde{B}_{k_{1}, \ldots, k_{p}}^{(\beta \gamma)}=B_{k_{1}, \ldots, k_{p}}^{(\beta \gamma)}-\sum_{i=1}^{p} l_{k_{i}}^{(\beta)} l(\gamma)_{k_{i}}\left[\pi^{2}-\psi^{\prime}\left(m_{k_{i}}\right)\right] .
\end{aligned}
$$

The last ingredients we need are the pole structures of $\Gamma$ and $\psi$ for $m \in \mathbb{Z}_{>}$:

$$
\begin{aligned}
\Gamma(1-m) & =\frac{1}{\Gamma(m)} \frac{\pi}{\sin (\pi m)}, \\
\psi(1-m) & =\psi(m)+\pi \cot (\pi m) \\
\psi^{\prime}(1-m) & =-\psi^{\prime}(m)+\pi^{2}\left[1+\cot ^{2}(\pi m)\right] \\
\psi^{\prime \prime}(1-m) & =\psi^{\prime \prime}(m)+2 \pi^{3} \cot (\pi m)+2 \pi^{3} \cot ^{3}(\pi m)
\end{aligned}
$$

We now consider the cases (I), (II) and (III) in turn:

(I) If we have more than one $-m_{k}<0$, then $c(n)$ has a double zero. $A^{(\beta)}$, however, only has a simple pole, i.e. we have to consider only the case where $-m_{k}<0$ for one $k$ only. Then

$$
\begin{aligned}
& c(n)=\frac{\prod_{j}\left(\Sigma_{\alpha} l_{0_{j}}^{(\alpha)} n_{\alpha}\right) !}{\prod_{i \neq k}\left(\Sigma_{\alpha} l_{i}^{(\alpha)} n_{\alpha}\right) !}\left(m_{k}-1\right) ! \frac{1}{\pi} \sin \left(\pi m_{k}\right) \\
& \begin{aligned}
A^{(\beta)}(n) & =-l_{k}^{(\beta)} \psi\left(1-m_{k}\right)+\text { finite } \\
& =-\pi l_{k}^{(\beta)} \cot \left(\pi m_{k}\right)+\text { finite }
\end{aligned}
\end{aligned}
$$

and we get

$$
\partial_{\beta} c(n)=-(-1)^{m_{k}} l_{k}^{(\beta)} \frac{\prod_{j}\left(\sum_{\alpha} l_{0_{j}}^{(\alpha)} n_{\alpha}\right) !\left(m_{k}-1\right) !}{\prod_{i \neq k}\left(\sum_{\alpha} l_{i}^{(\alpha)} n_{\alpha}\right) !} m_{k} \in \mathbb{Z}>.
$$

(II) Since $A^{2}$ and $B$ have double poles, we have to distinguish two possibilities:

(i) $-m_{k}<0$ for one $k$ only,

(ii) $-m_{k}<0$ for $k_{1}$ and $k_{2}$.

For the two cases we find respectively

$$
\partial_{\beta} \partial_{\gamma} c(n)=-(-1)^{m_{k}} \frac{\Pi_{j}\left(\sum_{\alpha} l_{0_{j}}^{(\alpha)} n_{\alpha}\right) !\left(m_{k}-1\right) !}{\prod_{i \neq k}\left(\sum_{\alpha} l_{i}^{(\alpha)} n_{\alpha}\right) !}\left(\tilde{A}_{k}^{(\beta)} l_{k}^{(\gamma)}+\tilde{A}_{k}^{(\gamma)} l_{k}^{(\beta)}\right)
$$

and

$$
\begin{aligned}
\partial_{\beta} \partial_{\gamma} c(n)= & (-1)^{m_{k_{1}}+m_{k_{2}}} \frac{\prod_{j}\left(\sum_{\alpha} l_{0 j}^{(\alpha)} n_{\alpha}\right) !\left(m_{k_{1}}-1\right) !\left(m_{k_{2}}-1\right) !}{\prod_{i \neq k_{1}, k_{2}}\left(\sum_{\alpha} l_{i}^{(\alpha)} n_{\alpha}\right) !} \\
& \times\left(l_{k_{1}}^{(\beta)} l_{k_{2}}^{(\gamma)}+l_{k_{1}}^{(\gamma)} l_{k_{2}}^{(\beta)}\right) .
\end{aligned}
$$


(III) We now have to distinguish three cases:

(i) $-m_{k}<0$ for one $k$ only,

(ii) $-m_{k}<0$ for $k_{1}$ and $k_{2}$,

(iii) $-m_{k}<0$ for $k_{1}, k_{2}$ and $k_{3}$.

For the three cases we find respectively

$$
\begin{aligned}
\partial_{\beta} \partial_{\gamma} \partial_{\delta} c(n)= & -(-1)^{m_{k}} \frac{\prod_{j}\left(\sum_{\alpha} l_{0_{j}}^{(\alpha)} n_{\alpha}\right) !\left(m_{k}-1\right) !}{\prod_{i \neq k}\left(\sum_{\alpha} l_{i}^{(\alpha)} n_{\alpha}\right) !} \\
& \times\left[2 \pi^{2} l_{k}^{(\beta)} l_{k}^{(\gamma)} l_{k}^{(\delta)}+\left(\tilde{B}_{k}^{(\beta \gamma)} l_{k}^{(\delta)}+\tilde{B}_{k}^{(\gamma \delta)} l_{k}^{(\gamma)}+\tilde{B}_{k}^{(\delta \beta)} l_{k}^{(\gamma)}\right)\right. \\
& \left.+\left(\tilde{A}_{k}^{(\beta)} \tilde{A}_{k}^{(\gamma)} l_{k}^{(\delta)}+\tilde{A}_{k}^{(\gamma)} \tilde{A}_{k}^{(\delta)} l_{k}^{(\beta)}+\tilde{A}_{k}^{(\delta)} \tilde{A}_{k}^{(\beta)} l_{k}^{(\gamma)}\right)\right], \\
\partial_{\beta} \partial_{\gamma} \partial_{\delta} c(n)= & (-1)^{m_{k_{1}}+m_{k_{2}}} \frac{\prod_{j}\left(\sum_{\alpha} l_{0_{j}}^{(\alpha)} n_{\alpha}\right) !\left(m_{k_{1}}-1\right) !\left(m_{k_{2}}-1\right) !}{\prod_{i \neq k_{1}, k_{2}}\left(\sum_{\alpha} l_{i}^{(\alpha)} n_{\alpha}\right) !} \\
& \times\left[\tilde{A}_{k_{1}, k_{2}}^{(\beta)}\left(l_{k_{1}}^{(\gamma)} l_{k_{2}}^{(\delta)}+l_{k_{1}}^{(\delta)} l_{k_{2}}^{(\gamma)}\right)+\tilde{A}_{k_{1}, k_{2}}^{(\gamma)}\left(l_{k_{1}}^{(\delta)} l_{k_{2}}^{(\beta)}+l_{k_{1}}^{(\beta)} l_{k_{2}}^{(\delta)}\right)\right. \\
& \left.+\tilde{A}_{k_{1}, k_{2}}^{(\delta)}\left(l_{k_{1}}^{(\beta)} l_{k_{2}}^{(\gamma)}+l_{k_{1}}^{(\gamma)} l_{k_{2}}^{(\beta)}\right)\right]
\end{aligned}
$$

and

$$
\begin{aligned}
\partial_{\beta} \partial_{\gamma} \partial_{\delta} c(n)= & -(-1)^{m_{k_{1}}+m_{k_{2}}+m_{k_{3}}} \\
& \times \frac{\prod_{j}\left(\sum_{\alpha} l_{0_{j}}^{(\alpha)} n_{\alpha}\right) !\left(m_{k_{1}}-1\right) !\left(m_{k_{2}}-1\right) !\left(m_{k_{3}}-1\right) !}{\prod_{i+k_{1}, k_{2}, k_{3}}\left(\sum_{\alpha} l_{i}^{(\alpha)} n_{\alpha}\right) !} \\
& \times\left(l_{k_{1}}^{(\beta)} l_{k_{2}}^{(\gamma)} l_{k_{3}}^{(\delta)}+l_{k_{1}}^{(\gamma)} l_{k_{2}}^{(\delta)} l_{k_{3}}^{(\beta)}+l_{k_{1}}^{(\delta)} l_{k_{2}}^{(\beta)} l_{k_{3}}^{(\gamma)}\right. \\
& \left.+l_{k_{1}}^{(\gamma)} l_{k_{2}}^{(\beta)} l_{k_{3}}^{(\delta)}+l_{k_{1}}^{(\beta)} l_{k_{2}}^{(\delta)} l_{k_{3}}^{(\gamma)}+l_{k_{1}}^{(\delta)} l_{k_{2}}^{(\gamma)} l_{k_{3}}^{(\beta)}\right)
\end{aligned}
$$

\section{Appendix B}

Predicted numbers of lines for complete intersections in $\mathbb{P}^{3} \times \mathbb{P}^{3}$ and $\mathbb{P}^{3} \times \mathbb{P}^{2}$

For complete intersections in $\mathbb{P}^{3} \times \mathbb{P}^{3}$,

$$
\left(\begin{array}{l||lll}
\mathbb{P}^{3} & s_{1} & s_{2} & s_{3} \\
\mathbb{P}^{3} & t_{1} & t_{2} & t_{3}
\end{array}\right),
$$

there are eight possible (nontrivial) configurations: $\left(s_{1}, s_{2}, s_{3} \mid t_{1}, t_{2}, t_{3}\right)=(3,0$, $1 \mid 0,3,1),(0,2,2 \mid 2,2,0),(2,1,1 \mid 2,1,1),(2,1,1 \mid 1,2,1),(2,1,1 \mid 0,3,1)$, $(2,1,1 \mid 0,2,2),(3,1,0 \mid 0,2,2),(2,2,0 \mid 0,0,4)$. For all of these, the predicted 
numbers of lines with bi-degree $\left(n_{1}, n_{2}\right)$ with respect to $J_{1}$ and $J_{2}$ from $\mathbb{P}^{3}$ and $\mathbb{P}^{3}$, respectively, are given generally by

$$
\begin{aligned}
N(1,0)= & 10 t_{1} t_{2} t_{3} s_{1} ! s_{2} ! s_{3} !-s_{1} ! s_{2} ! s_{3} ! \\
& \times\left[\frac{1}{2}\left(s_{2} s_{3} t_{1}^{3}+3 s_{1} s_{3} t_{1}^{2} t_{2}+3 s_{1} s_{2} t_{1}^{2} t_{3}+3 s_{1}^{2} t_{1} t_{2} t_{3}\right)\left(\sum_{r=1}^{s_{1}} \frac{1}{r^{2}}\right)\right. \\
& +4\left(s_{3} t_{1}^{2} t_{2}+s_{2} t_{1}^{2} t_{3}+2 s_{1} t_{1} t_{2} t_{3}\right)\left(\sum_{r=1}^{s_{1}} \frac{1}{r}\right) \\
& -\frac{1}{2}\left(s_{2} s_{3} t_{1}^{3}+3 s_{1} s_{3} t_{1}^{2} t_{2}+3 s_{1} s_{2} t_{1}^{2} t_{3}+3 s_{1}^{2} t_{1} t_{2} t_{3}\right)\left(\sum_{r=1}^{s_{1}} \frac{1}{r}\right)^{2} \\
& -\left(2 s_{2} s_{3} t_{1}^{2} t_{2}+2 s_{1} s_{3} t_{1} t_{2}^{2}+s_{2}^{2} t_{1}^{2} t_{3}+4 s_{1} s_{2} t_{1} t_{2} t_{3}+s_{1}^{2} t_{2}^{2} t_{3}\right) \\
& \times\left(\sum_{r=1}^{s_{1}} \frac{1}{r}\right)\left(\sum_{r=1}^{s_{2}} \frac{1}{r}\right) \\
& \left.+\left(\text { cyclic permutations: }\left(s_{1}, t_{1}\right) \rightarrow\left(s_{2}, t_{2}\right) \rightarrow\left(s_{3}, t_{3}\right)\right)\right]
\end{aligned}
$$

and by $N(0,1)=\left.N(1,0)\right|_{\left(s_{i} \leftrightarrow t_{i}\right)}$.

The predictions for seven possible nontrivial examples in $\mathbb{P}^{3} \times \mathbb{P}^{2},\left(s_{1}, s_{2} \mid t_{1}, t_{2}\right)$ $=(2,2 \mid 0,3),(3,1 \mid 0,3),(2,2 \mid 1,2),(4,0 \mid 1,2),(1,3 \mid 2,1),(3,1 \mid 2,1),(0,4 \mid 3,0)$, can be extracted from the above general formulas via the manifold identity,

$$
\left(\begin{array}{l}
\mathbb{P}^{3} \\
\mathbb{P}^{2}
\end{array} \mid \begin{array}{ll}
s_{1} & s_{2} \\
t_{1} & t_{2}
\end{array}\right) \cong\left(\begin{array}{l}
\mathbb{P}^{3} \\
\mathbb{P}^{3}
\end{array} \| \begin{array}{lll}
s_{1} & s_{2} & 0 \\
t_{1} & t_{2} & 1
\end{array}\right) .
$$

Further reduction simply reproduces the result for the bi-cubic model in $\mathbb{P}^{2} \times \mathbb{P}^{2}$ and $(4 \mid 2)$ in $\mathbb{P}^{3} \times \mathbb{P}^{1}$ which are treated in Section 7.

\section{References}

[1] T. Hübsch, Calabi-Yau manifolds: A bestiary for physicists, (World Scientific, Singapore, 1992).

[2] P. Green and T. Hübsch, Commun. Math. Phys. 113 (1987) 99.

[3] P. Candelas, A.M. Dale, C.A. Lütken and R. Schimmrigk, Nucl. Phys. B 298 (1988) 493.

[4] P. Candelas, C.A. Lütken and R. Schimmrigk, Nucl. Phys. B 306 (1988) 113.

[5] P. Green, T. Hübsch and C.A. Lütken, Class. Quantum Grav. 6 (1989) 105.

[6] C.T.C. Wall, Invent. Math. 1 (1966) 355.

[7] A. He and P. Candelas, Commun. Math. Phys. 135 (1990) 193.

[8] P. Green and T. Hübsch, Commun. Math. Phys. 119 (1988) 441.

[9] S.T. Yau, in Anomalies, geometry and topology, Proc. Symp., Argonne, Illinois, 1985, eds. W.A. Bardeen and A.R. White (World Scientific, Singapore 1985), with appendix of G. Tian and S.T. Yau.

[10] R. Schimmrigk, Phys. Lett. B 193 (1987) 175. 
[11] P. Candelas, X. De la Ossa, P. Green and L. Parkes, Nucl. Phys. B 359 (1991) 21.

[12] D. Morrison, Picard-Fuchs equations and mirror maps for hypersurfaces, in Essays on mirror manifolds, ed. S.-T. Yau (International Press, Hong Kong, 1992).

[13] A. Klemm and S. Theisen, Nucl. Phys. B 389 (1993) 153, and Theor. Math. Phys. 95 (1993) 583.

[14] A. Font, Nucl. Phys. B 391 (1993) 358.

[15] P. Candelas, X. de la Ossa, A. Font, S. Katz and D. Morrison, Nucl. Phys. B416 (1994) 481. P. Candelas, A. Font, S. Katz and D. Morrison, Nucl. Phys. B429 (1994) 626.

[16] S. Hosono, A. Klemm, S. Theisen and S.-T. Yau: Mirror symmetry, mirror map and applications to Calabi-Yau hypersurfaces, preprint HUTMP-93/0801, LMU-TPW-93-22 [hep-th/9308122], Commun. Math. Phys., to be published.

[17] P. Berglund, P. Candelas, X. de la Ossa, A. Font, T. Hübsch, D. Jancic and F. Quevedo, Nucl. Phys. B419 (1994) 352.

[18] A. Libgober and J. Teitelbaum, Duke Math. J., Int. Math. Res. Notices 1 (1993) 29.

[19] A. Klemm and S. Theisen, Mod. Phys. Lett. A9 (1994) 1807.

[20] V. Batyrev and D. van Straten, Generalized hypergeometric functions and rational curves on Calabi-Yau complete intersections in toric varieties, Essen Preprint (1993).

[21] D.E. Sommervoll, Rational curves of low degree on a complete intersection Calabi-Yau threefold in $\mathrm{P}^{3} \times \mathrm{P}^{3}$, Oslo preprint ISBN 82-553-0838-5.

[22] M. Bershadsky, S. Ceccotti, H. Ooguri and C. Vafa, Nucl. Phys. B 405 (1993) 279.

[23] M. Bershadsky, S. Ceccotti, H. Ooguri and C. Vafa, Commun. Math. Phys. 165 (1994) 311.

[24] J. Louis and V. Kaplunovski, in preparation.

[25] D. Dais and A. Klemm, On the invariants of minimal desingularizations of 3-dimensional Calabi-Yau weighted complete intersection varieties, in preparation.

[26] V. Batyrev, Dual polyhedra and mirror symmetry for Calabi-Yau hypersurfaces in toric varieties, J. Alg. Geom. to appear; Duke Math. J. 69 (1993) 349.

[27] L. Borisov, Towards the mirror symmetry for Calabi-Yau complete intersections in Gorenstein toric Fano varieties, Ann Arbor preprint (1993) [alg-geom/9310005].

[28] M. Yoshida, Fuchsian differential equations (Vieweg, Braunschweig, 1987).

[29] P.A. Griffiths, Bull. Am. Math. Soc. 76 (1970) 228.

[30] L. Billera, P. Filliman and B. Sturmfels, Adv. Math. 83 (1990) 155; T. Oda and H.S. Park, Tôhoku Math. J. 43 (1991) 375.

[31] P.S. Aspinwall, B.R. Greene and D. Morrison, Phys. Lett. B 303 (1993) 249; Nucl. Phys. B 416 (1994) 414.

[32] S. Hosono, A. Klemm and S. Theisen, Lectures on mirror symmetry, preprint HUTMP-94/01, LMU-TPW-94-02.

[33] P. Deligne, Equations différentielles à point singulieres régulieres, Lecture notes of mathematics, Vol. 163 (Springer, Berlin, 1970).

[34] R.L. Bryant and P.A. Griffith, in Progress in mathemathics, Vol. 36, Arithmetic and geometry (Birkhauser, Basel, 1983) pp. 77-102.

[35] P. Candelas and X. de la Ossa, Nucl. Phys. B 355 (1991) 455.

[36] Gelfand, Kapranov and Zelevinski, Leningrad Math. J. 2 (1991) 449.

[37] T. Todorov, private communication.

[38] D. Morrison, Compactifications of moduli spaces inspired by mirror symmetry, Duke preprint DUK-M93-06 (1993).

[39] P. Candelas, X. de la Ossa, Nucl. Phys. B 355 (1991) 455.

[40] E. Witten, Commun. Math. Phys. 117 (1988) 353; 118 (1988) 411;

T. Eguchi and S.K. Yang, Int. J. Mod. Phys. A 5 (1990) 1693;

R. Dijkgraaf, H. Verlinde and E. Verlinde, Nucl. Phys. B 352 (1991) 59.

[41] M. Kontsevich and Yu. Manin, Gromov-Witten classes, quantum cohomology, and enumerative geometry, Max-Planck-Institut für Mathematik preprint, Bonn (1994).

[42] B. de Wit and A. Van Proeyen, Nucl. Phys. B 245 (1984) 89;

B. de Wit, P. Lauwers and A. Van Proeyen, Nucl. Phys. B 255 (1985) 569.

[43] N. Seiberg, Nucl. Phys. B 303 (1988) 286. 
[44] S. Cecotti, S. Ferrara and L. Girardello, Int. J. Mod. Phys. A 4 (1989) 2475;

S. Ferrara, Nucl. Phys. (Proc. Suppl.) 11 (1989) 342.

[45] L. Dixon, V. Kaplunovsky and J. Louis, Nucl. Phys. B 329 (1990) 27.

[46] A. Ceresole, R. D'Auria, S. Ferrara, W. Lerche and J. Louis, Int. J. Mod. Phys. A 8 (1993) 79.

[47] E. Witten, in Essays on mirror manifolds, ed. S.T. Yau (International Press. Hong Kong, 1992).

[48] P. Aspinwall and D. Morrison, Commun. Math. Phys. 151 (1993) 45.

[49] S. Kalara and R.N. Mohapatra, Phys. Rev. D 36 (1987) 3474.

[50] B.R. Greene, K.H. Kirklin, P.J. Miron and G.G. Ross, Nucl. Phys. B 278 (1986) 667.

[51] D.E. Sommervoll, private communication.

[52] D. Gepner, Phys. Lett. B 199 (1987) 380; Nucl. Phys. B 296 (1988) 757; B 311 (1988) 191.

[53] A. Cappelli, C. Itzyson and B. Zuber, Commun. Math. Phys. 113 (1987) 1;

D. Gepner, Nucl. Phys. B 287 (1987) 111.

[54] G. Sotkov and M. Stanishkov, Phys. Lett. B 215 (1988) 647;

A. Kato and Y. Katizawa, Nucl. Phys. B 319 (1989) 474;

A. Klemm and J. Fuchs, Ann. Phys. 194 (1989) 303;

S. Cordes and Y. Kikuchi, Texas A\&M preprint CTP-TAMU-92/88.

[55] J. Fuchs, A. Klemm, C. Scheich and M. Schmidt, Ann. Phys. 204 (1990) 1.

[56] B. Greene, C. Vafa and N. Warner, Nucl. Phys. B 324 (1989) 371.

[57] E. Witten, Nucl. Phys. B 403 (1993) 159.

[58] P. Candelas, E. Derrick and L. Parkes, Nucl. Phys. B 407 (1993) 115.

[59] S. Cecotti and C. Vafa, Nucl. Phys. B 367 (1991) 359.

[60] S. Hosono, A. Klemm and S. Theisen, unpublished.

[61] B. Greene, R. Plessner and D. Morrison, Mirror manifolds in higher dimension, preprint CLNS-93/1253, IASSNS-HEP-94/2, YCTP-P31-92 [hep-th/9402119].

[62] M. Kreuzer and H. Skarke, Nucl. Phys. B 388 (1993) 113;

A. Klemm and R. Schimmrigk, Nucl. Phys. B 411 (1994) 559.

[63] B. Dubrovin, Nucl. Phys. B 379 (1992) 627. 